\author{
Universidade de São Paulo \\ Instituto de Física
}

\title{
Aspectos das transformações conformes na eletrodinâmica: invariância e leis de conservação
}

\author{
Vaguiner Rodrigues dos Santos
}

Orientador: Prof. Dr. Ruy Pepe da Silva

Dissertação de mestrado apresentada ao Instituto de Física para a obtenção do título de Mestre em Ciências

Banca Examinadora:

Prof. Dr. Ruy Pepe da Silva (IFUSP)

Prof. Dr. André Koch Torres Assis (UNICAMP)

Prof. Dr. Francisco Eugênio Mendonça da Silveira (UFABC) 


\section{FICHA CATALOGRÁFICA}

Preparada pelo Serviço de Biblioteca e Informação do Instituto de Física da Universidade de São Paulo

\section{Santos, Vaguiner Rodrigues dos}

Aspectos das transformações conformes na eletrodinâmica: invariância e leis de conservação - São Paulo, 2013.

Dissertação (Mestrado) - Universidade de São Paulo. Instituto de Física - Depto. de Física Aplicada

Orientador: Prof. Dr. Ruy Pepe da Silva

Área de Concentração: Física

Unitermos: 1.Eletrodinâmica; 2. Teoria dos Campos;

3. Transformações Conformes; 4. Leis de Conservação;

5. Invariância 
Não há ramo na matemática, por abstrato que seja, que não possa um dia vir a ser aplicado aos fenômenos do mundo real.

Nikolai Lobachevsky

O cientista não estuda a natureza porque ela é útil; estuda-a porque se delicia com ela, e se delicia com ela porque ela é bela. Se a natureza não fosse bela, não valeria a pena conhecê-la e, se não valesse a pena conhecê-la, não valeria a pena viver.

Henri Poincaré 

Dedico este trabalho aos meus pais, Davi e Antonia, e às minhas irmãs, Ivani e Vania, minha preciosa família. 



\section{AGRADECIMENTOS}

Ao Prof. Dr. Ruy Pepe da Silva, por sua orientação e pela sua compreensão. Agradeço pela confiança depositada em mim e pela condução do trabalho desde as primeiras discussões até a confecção final desta dissertação.

Um agradecimento especial ao Prof. Dr. Alvaro Vannucci, pelo incentivo que me deu quando estava iniciando os estudos que levaram à realização deste trabalho e pela atenção dispensada durante este período.

Ao pessoal da biblioteca pelo apoio dado na obtenção do material bibliográfico.

A todos que contribuíram para a confecção desta dissertação. 



\section{RESUMO}

Neste trabalho, discutem-se aspectos das transformações conformes na eletrodinâmica clássica com ênfase na invariância e nas leis de conservação. Inicialmente, abordaram-se aspectos gerais das transformações conformes e fez-se um resumo histórico da evolução dessas transformações. Procurou-se fazer uma apresentação didática, revisando-se a formulação Lagrangiana e o Teorema de Noether para campos aplicado à eletrodinâmica. Estudaram-se as transformações conformes no espaço plano, onde se mostrou que para dimensões $n \geq 3$ o número de transformações é finito. A partir das equações de Maxwell em coordenadas curvilíneas, chegou-se à condição para que essas equações mantivessem sua forma cartesiana. Com essa condição, mostrou-se que a eletrodinâmica clássica é invariante para o grupo de transformações conformes. Foram discutidas as leis de conservação associadas à invariância conforme da eletrodinâmica clássica a partir do teorema de Noether. Das simetrias por translações no espaço-tempo, obtiveram-se as leis de conservação do momento linear e da energia. Das simetrias associadas às rotações, obtiveram-se seis quantidades conservadas: três delas ligadas à conservação do momento angular e, com relação às três restantes, observou-se, a partir de analogias com a mecânica, que estavam associadas ao movimento do centro de energia do campo. Para a interpretação da grandeza conservada por simetria de escala, verificou-se, também a partir de uma analogia mecânica, que essa simetria somente é verificada para partículas não massivas ou para partículas massivas a altas energias. Finalmente, para as transformações conformes especiais, verificou-se que as leis de conservação resultantes são consequências das leis anteriores de conservação para o campo eletromagnético, e neste caso, essa simetria também somente se manifesta para partículas de massa nula ou para altas energias. 



\begin{abstract}
In this work, aspects of conformal transformations in classical electrodynamics are discussed with emphasis on the invariance and conservation laws. Initially, a general view of conformal transformations was shown and a summary of the historical evolution of those transformations was presented. The work was approached didactically, and Noether's theorem based on the electrodynamics Lagrangian formulation was revised. The conformal transformations were studied in plane spaces and it was shown that, for dimension $n \geq 3$, the number of transformations is finite. Starting from Maxwell's equations in curvilinear coordinates, a condition for maintaining those equations in Cartesian form was established. With that condition, it was shown that the classical electrodynamics laws are invariant for the group of conformal transformations. The conservation laws associated with the conformal invariance of classical electrodynamics were discussed, based on Noether's theorem. From the space-time translation symmetry, the laws of conservation of linear momentum and of energy were obtained. From rotational symmetry, six conserved quantities were obtained: three of them associated with angular momentum and the remaining three, observed, starting from analogies with mechanics, were associated with the movement of the center of energy of the field. For the interpretation of the quantity conserved by scale symmetry, it was verified, also from a mechanical analogy, that that symmetry is only valid for null mass particles or for high energies. Finally, for the special conformal transformations, it was verified that the resultant laws of conservation are consequences of the previous laws, and in that case, symmetry is also valid only for particles of null mass or for high energies.
\end{abstract}





\section{SUMÁRIO}

CAPÍTULO I - INTRODUÇÃO 1

CAPÍTULO II - TRANSFORMAÇÕES CONFORMES: ASPECTOS

GERAIS E SÍNTESE HISTÓRICA..................................................................

2.1 - Aspectos, definições e exemplos associados às transformações conformes................ 5

2.2 - Síntese da história das transformações conformes e suas aplicações..........................19

\section{CAPÍTULO III - A ELETRODINÂMICA CLÁSSICA E O}

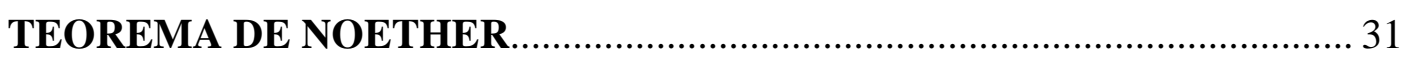

3.1 - Alguns resultados da eletrodinâmica covariante................................................ 31

3.2 - A formulação Lagrangiana para campos................................................................... 35

3.3 - A formulação Lagrangiana para o campo eletromagnético........................................ 39

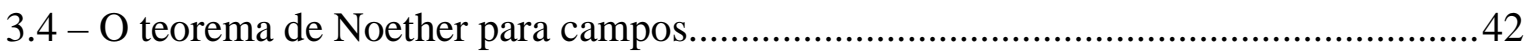

3.5 - Aplicação do teorema de Noether para o campo eletromagnético............................. 47

\section{CAPÍTULO IV - TRANSFORMAÇÕES CONFORMES EM}

ESPAÇOS PLANOS DE $N$ DIMENSÕES....................................................... 51

4.1 - Condições para as transformações conformes

em espaços planos de $n$ dimensões........................................................................51

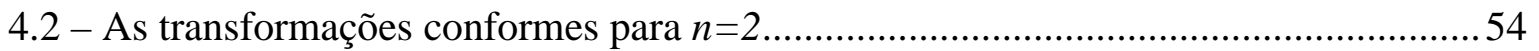

4.3 - As transformações conformes para $n \geq 3$.................................................. 55

4.4 - As transformações conformes no espaço-tempo....................................................... 59

4.5 - As transformações de Lorentz e de Poincaré................................................................62

\section{CAPÍTULO V - A INVARIÂNCIA CONFORME DAS}

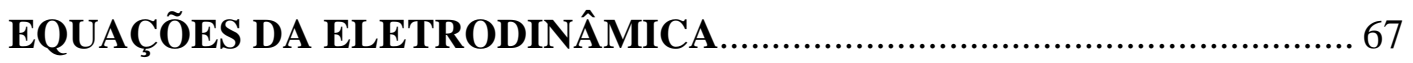

5.1 - As equações de Maxwell em coordenadas curvilíneas............................................ 67

5.2 - A invariância conforme das equações de Maxwell.................................................... 76 
CAPÍTULO VI - LEIS DE CONSERVAÇÃO ASSOCIADAS À INVARIÂNCIA CONFORME DO CAMPO ELETROMAGNÉTICO........................................ 79

6.1 - Leis de conservação associadas às translações no espaço-tempo...................................79

6.2 - Leis de conservação associadas às rotações no espaço-tempo...................................... 83

6.3 - Lei de conservação associada às dilatações no espaço-tempo.......................................89

6.4 - Leis de conservação associadas às transformações conformes

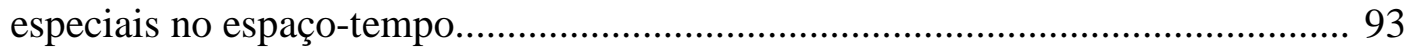

CAPÍTULO VII - RESULTADOS E CONCLUSÕES................................................97

APÊNDICE A - AS TRANSFORMAÇÕES CONFORMES ESPECIAIS

E O MOVIMENTO UNIFORMEMENTE ACELERADO................................ 101

APÊNDICE B - ALGUNS ASPECTOS DA TRANSFORMAÇÃO

DE DILATAÇÃO NA MECÂNICA CLÁSSICA................................................. 105

APÊNDICE C - AS TRANSFORMAÇÕES CONFORMES

ESPECIAIS NA MECÂNICA CLÁSSICA E RELATIVÍSTICA.......................109

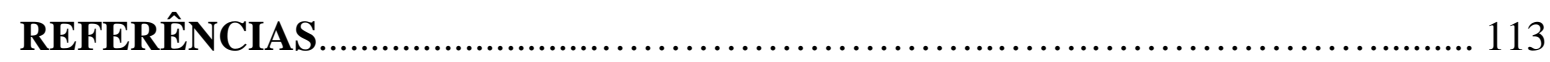




\section{CAPÍTULO I}

\section{INTRODUÇÃO}

Um fato importante na Física é que suas leis ou teorias são, em geral, expressas por equações diferenciais ou princípios variacionais. Essas leis e princípios devem preservar sua forma em diferentes sistemas de referência, ou seja, devem ser covariantes por uma mudança de coordenadas. O palco onde os fenômenos físicos ocorrem é o espaço e o tempo, e a forma como as coordenadas espaciais e a temporal se interligam é central na questão da covariância. O trabalho sobre a eletrodinâmica dos corpos em movimento de Albert Einstein (1879-1955) de 1905 [Ei-05] foi um dos marcos na história da Física. Nesse trabalho, são sintetizados vários fatos que já apontavam para uma nova forma de transformação de coordenadas entre sistemas inerciais que viria a substituir as transformações de Galileu ligadas à Mecânica Clássica. As novas transformações, onde o espaço e o tempo estavam interligados, ficaram conhecidas como transformações de Lorentz, pois foram propostas por esse físico antes de 1905. Essas transformações mantêm invariantes as equações da eletrodinâmica de Maxwell.

Outro aspecto importante nas teorias físicas são as leis de conservação, ou seja, leis que expressam a conservação de certas grandezas nas transformações de coordenadas, as chamadas simetrias. O nome ligado a essas leis e simetrias é o de Emmy Noether (1882-1935), com o teorema que leva o seu nome, publicado em 1918 [No-18, Ta-71], onde são relacionadas simetrias com leis de conservação.

O presente trabalho está ligado a essas questões: transformações de coordenadas, covariâncias, simetrias e leis de conservação na eletrodinâmica. Nele, será estudada uma classe especial de transformações de coordenadas e invariâncias, as chamadas transformações e invariâncias conformes, onde os ângulos são preservados.

Em 1909, Harry Bateman (1882-1946) e Ebenezer Cunningham (1881-1977) apresentaram trabalhos $[\mathrm{Ba}-10, \mathrm{Cu}-10]$ que marcaram o início das aplicações das transformações conformes em teorias de campos. Estes artigos deram continuação aos resultados obtidos por Lorentz em 1904, que mostraram que as equações de Maxwell permaneciam invariantes pelas transformações de Poincaré.

Após as publicações dos trabalhos de Bateman e Cunningham, seguiram-se as discussões sobre as aplicações e, principalmente, sobre o significado físico da invariância conforme das equações de Maxwell. Dentre elas, está o trabalho de Erich Bessel-Hagen (1898-1946) de 1921 [Be-21, Al-06], que examina as leis de conservação associadas ao 
campo eletromagnético. O interesse por essas transformações se mantém até hoje [Ka-08, B1-09, Go-13].

Nesta dissertação, objetiva-se estudar a invariância conforme da eletrodinâmica de Maxwell bem como questões associadas ao significado físico dessa invariância no espaçotempo de Minkowski. Inicialmente, abordam-se as transformações conformes em vários espaços, desde o plano complexo até sua generalização para $n$ dimensões, com ênfase nas transformações no espaço-tempo. A seguir, demonstra-se a invariância das equações de Maxwell por estas transformações, e, finalmente, por meio do teorema de Noether, verificamse as leis de conservação associadas a essa invariância.

São os seguintes os tópicos abordados nos capítulos que se seguem.

No capítulo II, discutem-se os principais aspectos das transformações conformes em espaços de $n$ dimensões e são apresentados exemplos dessas transformações. É também feito um apanhado histórico do desenvolvimento dessas transformações desde os tempos gregos até o início da década de 1970, quando a invariância conforme passou a ser desenvolvida e aplicada em vários ramos da física, como, por exemplo, em física de partículas de altas energias e em Mecânica Estatística.

No capítulo III, faz-se, inicialmente, uma revisão da eletrodinâmica covariante, da formulação Lagrangiana para campos e da aplicação desse formalismo na eletrodinâmica. Segue-se uma apresentação do teorema de Noether e de sua aplicação ao campo eletromagnético livre.

No capítulo IV, determinam-se as transformações conformes em espaços planos de $n$ dimensões. Apresentam-se, inicialmente, as condições para que uma transformação infinitesimal de coordenadas seja conforme em um espaço plano $n$-dimensional. Como exemplo de aplicação, mostra-se o caso particular das transformações conformes em duas dimensões, e a seguir parte-se para a determinação das transformações infinitesimais conformes em espaços planos para dimensões iguais ou superiores a três. Finalmente, discutem-se aspectos das transformações de Lorentz-Poincaré.

No capítulo $\mathrm{V}$, discute-se a invariância conforme das equações da eletrodinâmica de Maxwell. Inicia-se com o conceito de derivada covariante em coordenadas curvilíneas e, a seguir, são apresentadas as equações de Maxwell nessas coordenadas no espaço-tempo plano. Finalmente, chega-se ao conjunto de transformações de coordenadas que mantêm as equações de Maxwell na mesma forma que elas se apresentam em coordenadas cartesianas.

No capítulo VI, obtêm-se as leis de conservação associadas à invariância conforme do campo eletromagnético livre. Nesse estudo, utiliza-se a equação de continuidade obtida 
com o teorema de Noether e juntamente com as transformações conformes obtidas no Capítulo IV determinam-se as leis de conservação associadas às simetrias ligadas a essas transformações. São também discutidos aspectos físicos associados a essas transformações.

No capítulo VII, apresentam-se os resultados e as conclusões do trabalho.

Neste trabalho, utiliza-se o sistema de unidades natural, onde se adota que a velocidade da luz no vácuo é dada por $c=1$. 


\section{CAPÍTULO II}

\section{TRANSFORMAÇÕES CONFORMES: ASPECTOS GERAIS E SÍNTESE HISTÓRICA.}

Neste capítulo, apresentam-se conceitos, algumas propriedades e exemplos de transformações conformes. Discutem-se, primeiramente, aspectos relevantes das transformações conformes e apresentam exemplos com o objetivo de tornar claros os conceitos utilizados. Parte-se, inicialmente, de transformações em duas dimensões (mapeamento conforme no plano complexo) para se chegar a transformações em espaços de $n$ dimensões, com especial atenção a $n=4$ (espaço-tempo de Minkowski). Em seguida, apresenta-se um resumo histórico do desenvolvimento dessas transformações, desde sua utilização na elaboração de mapas até aplicações na Física.

\subsection{Aspectos, definições e exemplos associados às transformações conformes.}

As transformações conformes no plano complexo, além de configurarem um campo à parte da matemática, são comumente utilizadas em Física e em Engenharia para a resolução de problemas bidimensionais nas áreas de condução de calor, movimento de fluidos, eletromagnetismo, etc. Nesses casos, os problemas podem ser resolvidos através das técnicas de mapeamento conforme, que se baseiam em propriedades das funções analíticas [Ar-85, Ha-99, Mc-03, Ne-97, Wu-05].

No plano complexo, seja uma transformação $\zeta \rightarrow w$ que transforma o ponto $\zeta(x, y)$ em $w(u, v)$. Essa transformação é representada por:

$$
w=f(\zeta)=u(x, y)+i v(x, y)
$$

Se a função $f(\zeta)$ é analítica em certa região do plano complexo $\zeta$, então $u(x, y)$ e $v(x, y)$ satisfazem as condições de Cauchy-Riemann:

$$
\frac{\partial u(x, y)}{\partial x}=\frac{\partial v(x, y)}{\partial y} \quad \text { e } \quad \frac{\partial u(x, y)}{\partial y}=-\frac{\partial v(x, y)}{\partial x}
$$


Se $f(\zeta)$ é analítica em $\zeta_{0}$ e se $f^{\prime}\left(\zeta_{0}\right) \neq 0$, tem-se que o mapeamento (2.1) é conforme em $\zeta_{0}$, ou seja, possui a propriedade de preservar o ângulo formado entre duas curvas que se interceptam nesse ponto [Ah-79]. Pode-se verificar essa propriedade considerando-se duas curvas, $C_{1}$ e $C_{2}$ (Fig. 2.1), que se interceptam em $\zeta_{0}$ no plano $\zeta$ e formam um ângulo $\theta$ entre si. O mapeamento dado pela função analítica $w=f(\zeta)$ transforma $C_{1}$ e $C_{2}$ nas curvas $C_{1}{ }^{\prime}$ e $C_{2}{ }^{\prime}$ no plano $w$ de tal modo que o ângulo $\theta^{\prime}$ formado entre $C_{1}{ }^{\prime}$ e $C_{2}{ }^{\prime}$ seja igual a $\theta$.
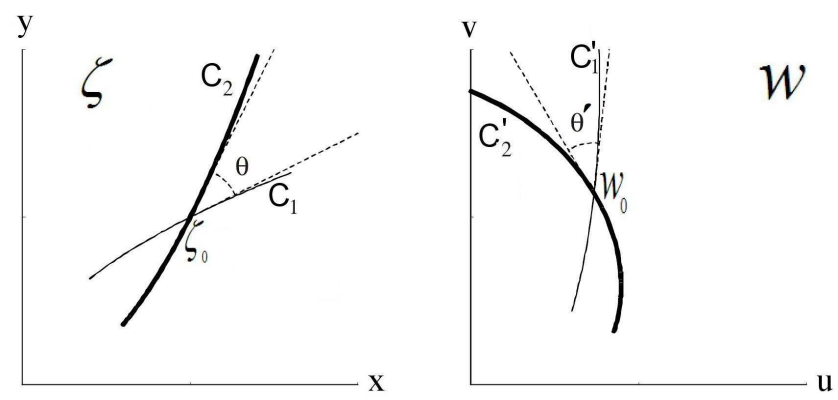

Fig. 2.1 - Representação de duas curvas $C_{1}$ e $C_{2}$ e das curvas transformadas, $C_{1}{ }^{\prime}$ e $C_{2}{ }^{\prime}$, no plano complexo. $\mathrm{O}$ ângulo formado entre as curvas nos planos $\zeta$ e $w$ não se altera se a transformação é conforme.

O ângulo $\theta$ formado entre as curvas $C_{1}$ e $C_{2}$ é dado pelos versores $\overrightarrow{e_{1}}$ e $\overrightarrow{e_{2}}$ tangentes a estas curvas no ponto $\zeta_{0}$, ou seja:

$$
\cos (\theta)=\frac{\overrightarrow{e_{1}} \cdot \overrightarrow{e_{2}}}{\left|\overrightarrow{e_{1}}\right| \cdot\left|\overrightarrow{e_{2}}\right|}=\overrightarrow{e_{1}} \cdot \overrightarrow{e_{2}}
$$

Um pequeno deslocamento $\vec{d}$ ao longo de uma curva $C$ em $\zeta$ é dado por:

$$
\vec{d}=(\Delta x, \Delta y)=\Delta x \overrightarrow{e_{x}}+\Delta y \overrightarrow{e_{y}}
$$

Assim, os versores tangentes às curvas $C_{1}$ e $C_{2}$ são dados respectivamente por: 


$$
\overrightarrow{e_{1}}=\frac{\overrightarrow{d_{1}}}{\left|\overrightarrow{d_{1}}\right|}=\frac{\Delta x_{1} \overrightarrow{e_{x}}+\Delta y_{1} \overrightarrow{e_{y}}}{\sqrt{\left(\Delta x_{1}\right)^{2}+\left(\Delta y_{1}\right)^{2}}} \quad \text { e } \quad \overrightarrow{e_{2}}=\frac{\overrightarrow{d_{2}}}{\left|\overrightarrow{d_{2}}\right|}=\frac{\Delta x_{2} \overrightarrow{e_{x}}+\Delta y_{2} \overrightarrow{e_{y}}}{\sqrt{\left(\Delta x_{2}\right)^{2}+\left(\Delta y_{2}\right)^{2}}}
$$

Deste modo, tem-se que os cossenos dos ângulos nos planos $\zeta$ e $w$ são dados, respectivamente, por:

$$
\cos (\theta)=\overrightarrow{e_{1}} \cdot \overrightarrow{e_{2}}=\frac{\Delta x_{1} \cdot \Delta x_{2}+\Delta y_{1} \cdot \Delta y_{2}}{\sqrt{\left(\Delta x_{1}\right)^{2}+\left(\Delta y_{1}\right)^{2}} \cdot \sqrt{\left(\Delta x_{2}\right)^{2}+\left(\Delta y_{2}\right)^{2}}}
$$

$\mathrm{e}$

$$
\cos \left(\theta^{\prime}\right)=\overrightarrow{e_{1}^{\prime}} \cdot \vec{e}_{2}^{\prime}=\frac{\Delta u_{1} \cdot \Delta u_{2}+\Delta v_{1} \cdot \Delta v_{2}}{\sqrt{\left(\Delta u_{1}\right)^{2}+\left(\Delta v_{1}\right)^{2}} \cdot \sqrt{\left(\Delta u_{2}\right)^{2}+\left(\Delta v_{2}\right)^{2}}},
$$

onde: $u=u(x, y)$ e $v=v(x, y)$.

Substituindo-se as relações:

$$
\Delta u_{m}=\frac{\partial u}{\partial x} \Delta x_{m}+\frac{\partial u}{\partial y} \Delta y_{m} \quad \text { e } \quad \Delta v_{m}=\frac{\partial v}{\partial x} \Delta x_{m}+\frac{\partial v}{\partial y} \Delta y_{m}
$$

para $m=1,2$, e as condições (2.2) nas relações $(2.3)$ e (2.4), obtém-se que $\overrightarrow{e_{1}} \cdot \overrightarrow{e_{2}}=\overrightarrow{e_{1}}{ }^{\prime} \cdot \overrightarrow{e_{2}}{ }^{\prime}$ ou $\theta=\theta^{\prime}$, mostrando que uma transformação definida por uma função analítica é conforme.

Para se mostrar a relação entre as métricas $d s^{2}=(d x)^{2}+(d y)^{2}$ em $\zeta$ e $d s^{\prime 2}=(d u)^{2}+(d v)^{2}$ em $w$, considera-se a derivada de $f(\zeta)$ no ponto $\zeta$, ou seja, $f^{\prime}(\zeta)$, e as condições (2.2), de onde se obtém o módulo ao quadrado de $f^{\prime}(\zeta)$, dado por:

$$
\left|f^{\prime}(\zeta)\right|^{2}=\left(\frac{\partial u}{\partial x}\right)^{2}+\left(\frac{\partial v}{\partial x}\right)^{2}=\left(\frac{\partial v}{\partial y}\right)^{2}+\left(\frac{\partial u}{\partial y}\right)^{2}
$$

Por outro lado, a transformação $w=f(\zeta)=u(x, y)+i v(x, y)$ implica em: 


$$
d w=d u+i d v=\left(\frac{\partial u}{\partial x} d x+\frac{\partial u}{\partial y} d y\right)+i\left(\frac{\partial v}{\partial x} d x+\frac{\partial v}{\partial y} d y\right)
$$

de onde se obtém:

$$
(d u)^{2}+(d v)^{2}=(d x)^{2}\left[\left(\frac{\partial u}{\partial x}\right)^{2}+\left(\frac{\partial v}{\partial x}\right)^{2}\right]+(d y)^{2}\left[\left(\frac{\partial u}{\partial y}\right)^{2}+\left(\frac{\partial v}{\partial y}\right)^{2}\right]+2 d x d y\left(\frac{\partial u}{\partial x} \frac{\partial u}{\partial y}+\frac{\partial v}{\partial x} \frac{\partial v}{\partial y}\right)
$$

Através das condições (2.2), vê-se que o terceiro termo do lado direito dessa igualdade se anula. Assim, utilizando-se a relação (2.5), obtém-se:

$$
(d u)^{2}+(d v)^{2}=\left|f^{\prime}(\zeta)\right|^{2}\left[(d x)^{2}+(d y)^{2}\right] \quad \text { ou } \quad d s^{\prime 2}=\left|f^{\prime}(\zeta)\right|^{2} d s^{2}
$$

onde: $\left|f^{\prime}(\zeta)\right|^{2} \neq 0$.

A expressão $d s^{\prime 2}=\left|f^{\prime}(\zeta)\right|^{2} d s^{2}$, como será visto mais adiante, é um caso particular de uma relação mais geral, válida para as transformações conformes em espaços de $n$ dimensões.

Também, a partir das condições de Cauchy-Riemann (2.2), verifica-se que $u(x, y)$ e $v(x, y)$ são funções harmônicas, isto é, satisfazem as equações de Laplace no plano $\zeta$ :

$$
\frac{\partial^{2} u(x, y)}{\partial x^{2}}+\frac{\partial^{2} u(x, y)}{\partial y^{2}}=0 \quad \text { e } \quad \frac{\partial^{2} v(x, y)}{\partial x^{2}}+\frac{\partial^{2} v(x, y)}{\partial y^{2}}=0
$$

A equação de Laplace aparece em muitos problemas físicos. O mais conhecido exemplo vem da eletrostática, onde a equação $\nabla^{2} \Phi(x, y, z)=0$ descreve o potencial numa região sem cargas. Neste caso, a função $\Phi(x, y, z)$ é conhecida numa superfície condutora e se quer determiná-la numa região interior ou exterior a esta superfície. Em muitas situações, as simetrias existentes reduzem o problema ao caso bidimensional, em que se determina a solução para o potencial $\Phi(x, y)$. Como $\Phi(x, y)$ é uma função harmônica, pode-se considerar 
que ela é a parte real de $F(\zeta)=\Phi(x, y)+i \psi(x, y)$, onde a função $F(\zeta)$ é denominada potencial complexo e $\psi(x, y)$ é a função harmônica conjugada de $\Phi(x, y)$.

Considerando-se os vetores normais às curvas de nível $\Phi(x, y)=$ constante e $\psi(x, y)=$ constante, e as condições (2.2), tem-se que o produto escalar desses vetores é nulo, ou seja:

$$
\vec{\nabla} \Phi \cdot \vec{\nabla} \psi=\frac{\partial \Phi}{\partial x} \frac{\partial \psi}{\partial x}+\frac{\partial \Phi}{\partial y} \frac{\partial \psi}{\partial y}=\frac{\partial \Phi}{\partial x}\left(-\frac{\partial \Phi}{\partial y}\right)+\frac{\partial \Phi}{\partial y}\left(\frac{\partial \Phi}{\partial x}\right)=0
$$

mostrando que as curvas de nível no plano $\zeta$, dadas pelas funções $\Phi(x, y)=$ constante e $\psi(x, y)=$ constante, são ortogonais entre si. Essa propriedade é útil, por exemplo, em eletrostática, para se obter a visualização das linhas do campo elétrico $\psi(x, y)=$ constante e das superfícies equipotenciais $\Phi(x, y)=$ constante em regiões sem carga. Em problemas bidimensionais que envolvam condução de calor, $\Phi(x, y)=$ constante representam isotermas e $\psi(x, y)=$ constante, linhas de fluxo térmico.

A técnica de mapeamento conforme é útil para a resolução de problemas bidimensionais devido à equação de Laplace ser invariante por transformações conformes no plano complexo. Considerando-se a equação de Laplace para a função $\Phi(x, y)$ no plano $\zeta$ :

$$
\nabla^{2} \Phi(x, y)=\frac{\partial^{2} \Phi(x, y)}{\partial x^{2}}+\frac{\partial^{2} \Phi(x, y)}{\partial y^{2}}=0
$$

tem-se que, através do mapeamento conforme $w=f(\zeta)$, existe uma função $\phi(u, v)$ que também é harmônica no plano $(u, v)$. Para se ter esse resultado, considera-se que a função $\phi(u, v)$ resulta da transformação conforme da função $\Phi(x, y)$, ou seja:

$$
\phi(u, v)=\Phi[x(u, v), y(u, v)] .
$$


Aplicando-se a regra da cadeia na relação acima, tem-se que as derivadas parciais de segunda ordem de $\Phi(x, y)$ em relação a $x$ e $y$ são dadas, respectivamente, por:

$\frac{\partial^{2} \Phi}{\partial x^{2}}=\frac{\partial \phi}{\partial u} \cdot \frac{\partial^{2} u}{\partial x^{2}}+\frac{\partial \phi}{\partial v} \cdot \frac{\partial^{2} v}{\partial x^{2}}+\frac{\partial u}{\partial x}\left[\frac{\partial^{2} \phi}{\partial u^{2}} \frac{\partial u}{\partial x}+\frac{\partial^{2} \phi}{\partial v \partial u} \frac{\partial v}{\partial x}\right]+\frac{\partial v}{\partial x}\left[\frac{\partial^{2} \phi}{\partial u \partial v} \frac{\partial u}{\partial x}+\frac{\partial^{2} \phi}{\partial v^{2}} \frac{\partial v}{\partial x}\right]$

$\mathrm{e}$

$\frac{\partial^{2} \Phi}{\partial y^{2}}=\frac{\partial \phi}{\partial u} \cdot \frac{\partial^{2} u}{\partial y^{2}}+\frac{\partial \phi}{\partial v} \cdot \frac{\partial^{2} v}{\partial y^{2}}+\frac{\partial u}{\partial y}\left[\frac{\partial^{2} \phi}{\partial u^{2}} \frac{\partial u}{\partial y}+\frac{\partial^{2} \phi}{\partial v \partial u} \frac{\partial v}{\partial y}\right]+\frac{\partial v}{\partial y}\left[\frac{\partial^{2} \phi}{\partial u \partial v} \frac{\partial u}{\partial y}+\frac{\partial^{2} \phi}{\partial v^{2}} \frac{\partial v}{\partial y}\right]$

Somando-se as expressões acima e utilizando-se as condições de Cauchy-Riemann (2.2), encontra-se:

$$
\frac{\partial^{2} \Phi}{\partial x^{2}}+\frac{\partial^{2} \Phi}{\partial y^{2}}=\left[\left(\frac{\partial v}{\partial x}\right)^{2}+\left(\frac{\partial u}{\partial x}\right)^{2}\right]\left[\frac{\partial^{2} \phi}{\partial u^{2}}+\frac{\partial^{2} \phi}{\partial v^{2}}\right]
$$

O primeiro termo do lado direito da expressão acima é igual a $\left|f^{\prime}(\zeta)\right|^{2}>0$, dado pela relação (2.5). Portanto, se $\nabla^{2} \Phi(x, y)=0$, tem-se que $\phi(u, v)$ também é harmônica, ou seja, $\nabla^{2} \phi(u, v)=0$.

Assim, um problema de contorno que é complicado no plano $\zeta$ pode ser tratado de uma forma mais simples no plano $w$, através do mapeamento conforme das variáveis $(x, y)$ nas variáveis $(u, v)$.

Examinam-se, agora, as transformações conformes em espaços de dimensão $n \geq 2$ [Ka-08].

Sejam $E^{n}$ e $\bar{E}^{n}$, dois espaços geométricos, isto é, espaços dotados de um tensor métrico $g_{\alpha \beta}$ que permite medidas de distâncias e ângulos. Pontos em $E^{n}$ e $\bar{E}^{n}$ têm, respectivamente, coordenadas dadas por $x^{\alpha}$ e $\bar{x}^{\mu}$, e tensores métricos $g_{\alpha \beta}(x)$ e $\bar{g}_{\mu v}(\bar{x})$. 
As distâncias entre dois pontos infinitesimalmente próximos em cada espaço são dadas, respectivamente, pelos elementos de linha:

$$
d s^{2}(x)=g_{\alpha \beta}(x) d x^{\alpha} d x^{\beta} \quad \text { e } \quad d s^{2}(\bar{x})=\bar{g}_{\mu v}(\bar{x}) d \bar{x}^{-\mu} d \bar{x}^{-v}
$$

A transformação do ponto $x$ no ponto $\bar{x}$ é conforme se os elementos de linha são relacionados por uma função escalar positiva $\sigma(x)>0$ (fator de escala), tal que [Be-84, Fu-62, Lo-77, Wo-89]:

$$
d s^{2}(\bar{x})=\sigma(x) d s^{2}(x)
$$

Mostrou-se, em (2.6), a relação entre as métricas numa transformação conforme no plano complexo. No caso das transformações conformes no plano complexo $(n=2)$, a relação acima se reduz à expressão (2.6), onde $\sigma(\zeta)=\left|f^{\prime}(\zeta)\right|^{2}>0$.

Em espaços de dimensão $n>2$, a preservação dos ângulos pode ser vista a partir da extensão da definição do cosseno do ângulo formado por duas curvas que se interceptam no ponto $x$ do espaço. Portanto, em $E^{n}$ tem-se que:

$$
\cos (\theta)=\frac{\overrightarrow{d \vec{x}_{1} \cdot d \vec{x}_{2}}}{\mid \overrightarrow{d x_{1}|\cdot| d \vec{x}_{2} \mid}}=\frac{g_{\mu v}(x) d x_{1}{ }^{\mu} d x_{2}{ }^{\nu}}{\sqrt{g_{\alpha \beta}(x) d x_{1}{ }^{\alpha} d x_{1} \beta} \sqrt{g_{\rho \sigma}(x) d x_{2}{ }^{\rho} d x_{2}{ }^{\sigma}}}
$$

onde $d x_{1}^{\mu}$ e $d x_{2}{ }^{v}$ são as coordenadas dos elementos de arco das duas curvas.

Considerando-se a mesma relação para $\bar{\theta}$ em $\bar{E}^{n}$, tem-se que:

$$
\cos (\bar{\theta})=\frac{\overrightarrow{\vec{x}_{1}} \cdot d \overrightarrow{\bar{x}}_{2}}{\left|\overrightarrow{\bar{x}_{1}}\right| \cdot\left|d \vec{x}_{2}\right|}=\frac{\bar{g}_{\gamma \delta}(\bar{x}) d \bar{x}_{1}^{\gamma} d \bar{x}_{2} \delta}{\sqrt{\bar{g}_{\kappa \lambda}(x) d \bar{x}_{1}{ }^{\kappa} d \bar{x}_{1} \lambda} \sqrt{\bar{g}_{\tau \eta}(\bar{x}) d \bar{x}_{2} \tau d \bar{x}_{2} \eta}}
$$

Como a transformação entre as curvas é conforme, de acordo com a relação (2.8) o produto escalar $d \overrightarrow{x_{1}} \cdot d \vec{x}_{2}$ é dado por: 


$$
d \overrightarrow{\bar{x}}_{1} \cdot d \overrightarrow{\bar{x}}_{2}=\bar{g}_{\gamma \delta}(\bar{x}) d \bar{x}_{1}^{\gamma} d \bar{x}_{2} \delta=\sigma(x) g_{\mu v}(x) d x_{1}^{\mu} d x_{2}{ }^{\nu}
$$

Utilizando-se a relação acima em (2.9), tem-se que a preservação dos ângulos é verificada, pois:

$$
\cos (\bar{\theta})=\frac{\sigma(x) g_{\mu \nu}(x) d x_{1}^{\mu} d x_{2}{ }^{\nu}}{\sqrt{\sigma(x) g_{\alpha \beta}(x) d x_{1}^{\alpha} d x_{1} \beta} \sqrt{\sigma(x) g_{\rho \sigma^{(}}(x) d x_{2}{ }^{\rho} d x_{2}{ }^{\sigma}}}=\cos (\theta) .
$$

A seguir, para ilustração e melhor clareza, apresenta-se um exemplo de transformação conforme historicamente importante e ligado à cartografia. Considera-se, aqui, a projeção estereográfica dos pontos $P(\xi, \eta, \chi)$, pertencentes à superfície de uma esfera de raio $a$, nos pontos $\widehat{P}(x, y)$ de um plano (Fig. 2.2) [Bl-00, Co-98, Ka-08, He-01, Ne-97]. Na figura, os eixos $x$ e $y$ coincidem respectivamente com os eixos $\xi$ e $\eta$. Nesta projeção, o polo norte da esfera (foco da projeção $\mathrm{N}$ ) está localizado no ponto $P(0,0,2 a)$, enquanto o pólo sul (S), $P(0,0,0)$, tangencia o plano e coincide com a origem das coordenadas do plano $(x, y)$. O mapeamento é feito a partir da reta determinada pelo polo norte da esfera e o ponto $P(\xi, \eta, \chi)$, sendo $\hat{\mathrm{P}}(\mathrm{x}, \mathrm{y})$ o ponto projetado no plano.

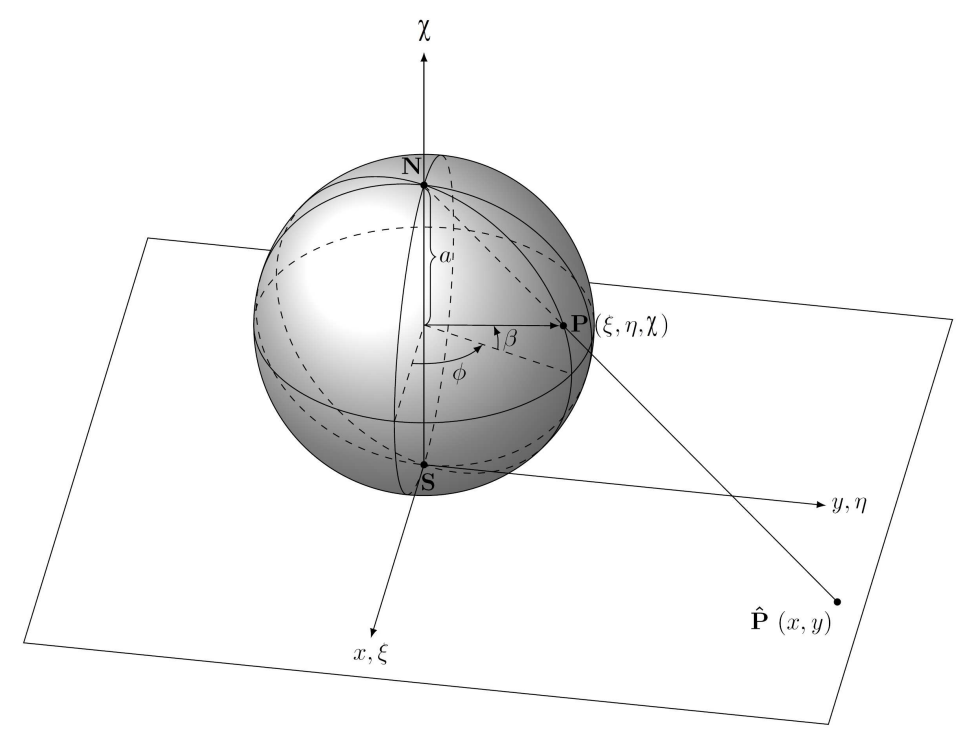

Fig. 2.2 - A projeção estereográfica em que os pontos de uma superfície esférica são transformados nos pontos de um plano tangente à superfície da esfera. [Ka-08] 
Um ponto $P(\xi, \eta, \chi)$ do espaço que pertença à superfície da esfera pode ser caracterizado pelas coordenadas $\varphi$ (longitude) e $\beta$ (latitude). As relações entre $(\xi, \eta, \chi)$ e $(\varphi, \beta)$ são dadas por:

$$
\xi=a \cos (\varphi) \cos (\beta), \quad \eta=a \operatorname{sen}(\varphi) \cos (\beta) \quad \text { e } \quad \chi-a=a \operatorname{sen}(\beta)
$$

Para esta projeção, as relações entre as coordenadas $(\varphi, \beta)$ e $(x, y)$ são dadas por:

$$
x=\frac{2 a \cos (\varphi) \cos (\beta)}{1-\operatorname{sen}(\beta)} \quad \text { e } \quad y=\frac{2 a \operatorname{sen}(\varphi) \cos (\beta)}{1-\operatorname{sen}(\beta)}
$$

Utilizando-se a identidade trigonométrica:

$$
\frac{\cos (\beta)}{1-\operatorname{sen}(\beta)}=\tan \left(\frac{\beta}{2}+\frac{\pi}{4}\right)
$$

nas relações para $x$ e $y$, obtém-se a seguinte relação entre os elementos de linha na superfície da esfera, $d s^{2}(\varphi, \beta)$, e no plano, $d s^{2}(x, y)$ :

$$
d s^{2}(x, y)=\frac{4}{[1-\operatorname{sen}(\beta)]^{2}} d s^{2}(\varphi, \beta)
$$

onde

$$
d s^{2}(x, y)=d x^{2}+d y^{2}, \quad d s^{2}(\varphi, \beta)=a^{2}\left[\cos ^{2}(\beta)(d \varphi)^{2}+(d \beta)^{2}\right]
$$
$\sigma(\varphi, \beta)=\frac{4}{[1-\operatorname{sen}(\beta)]^{2}}$ é o fator de escala.

Restringindo-se, agora, a mapeamentos num mesmo espaço, ou seja, onde os pontos $x$ e $\bar{x}$ estão no mesmo espaço, uma transformação de coordenadas é definida por funções reais, invertíveis e diferenciáveis $f\left(x^{\mu}\right)$, ou seja, $\bar{x}^{\mu}=f^{\mu}\left(x^{1}, x^{2}, \ldots, x^{n}\right)$, onde $\bar{x}^{\mu}$ são as 
coordenadas do ponto $\bar{x}$, e $x^{\mu}$, as do ponto $x$. As transformações das diferenciais $d x^{\alpha}$ são dadas por:

$$
d \bar{x} \mu=\frac{\partial \bar{x}^{\mu}}{\partial x^{\alpha}} d x^{\alpha}
$$

que substituídas em (2.7) e levando-se em conta (2.8), implicam na relação:

$$
\bar{g}_{\mu \nu}(\bar{x}) \frac{\partial \bar{x}^{-\mu}}{\partial x^{\alpha}} \frac{\partial \bar{x}^{-v}}{\partial x^{\beta}}=\sigma(x) g_{\alpha \beta}(x)
$$

A expressão acima e a relação (2.8) são formas alternativas para se definir uma transformação conforme.

Em alguns textos encontrados na literatura associada às transformações conformes depara-se com uma classificação dessas transformações em dois tipos diferentes, as ativas e as passivas. As primeiras são transformações entre pontos distintos de um mesmo espaço e as últimas são transformações que envolvem as coordenadas de um mesmo ponto do espaço representado em diferentes sistemas de coordenadas. Tanto no caso do mapeamento conforme (plano complexo) quanto no exemplo da projeção estereográfica representada na Fig. 2.2 temse transformações ativas.

As transformações passivas, isto é, envolvendo diferentes sistemas de coordenadas, são de grande interesse físico, pois envolvem situações em que um mesmo processo é descrito por dois observadores diferentes (dois sistemas de referência diferentes $S$ e $S^{\prime}$ ).

Para transformações entre dois referenciais $S$ e $S^{\prime}$ [Di-98], sejam $x^{\mu}$ e $x^{\prime \mu}$ as coordenadas de um mesmo ponto em relação a esses referenciais. Pode-se definir uma transformação (passiva) entre $S$ e $S^{\prime}$ pelas funções reais, invertíveis e diferenciáveis (equações de transformação):

$$
x^{\prime \mu}=h^{\prime \mu}\left(x^{1}, x^{2}, \ldots, x^{n}\right), \mu=1, \ldots n \text {. }
$$

O Jacobiano $J^{\prime}$ da transformação é dado por: 


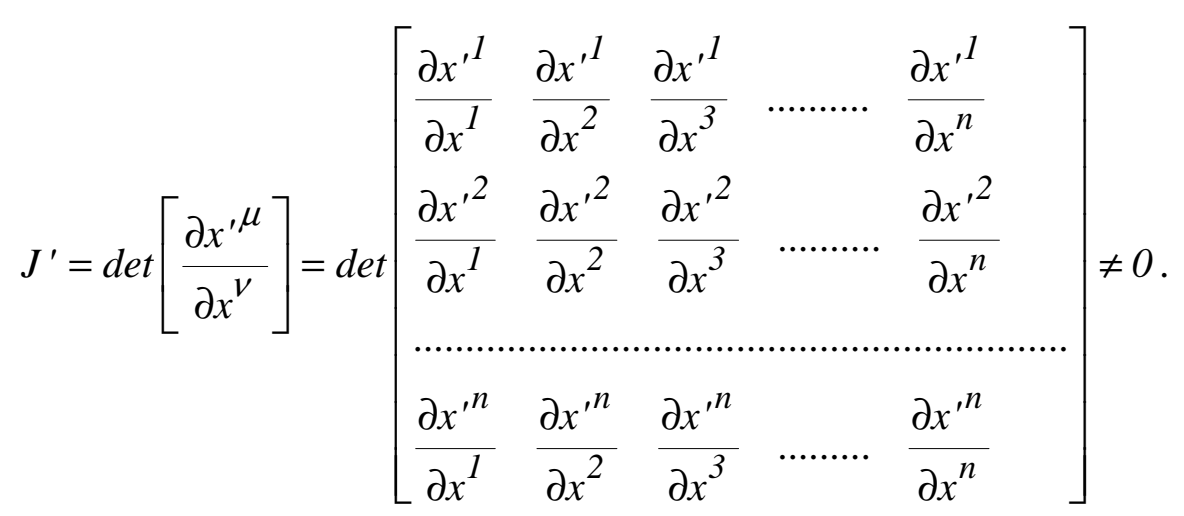

Das $n$ equações de transformação, $x^{\prime \mu}=h^{\prime \mu}\left(x^{\alpha}\right)$, têm-se:

$$
d x^{\prime \mu}=\frac{\partial x^{\prime \mu}}{\partial x^{\alpha}} d x^{\alpha}, \mu, \alpha=1, \ldots, n
$$

Sejam $g_{\alpha \beta}(x)$ e $g^{\prime}{ }_{\mu \nu}\left(x^{\prime}\right)$ os tensores métricos associados, respectivamente, a $S$ e $S^{\prime}$. Os elementos de linha em $S$ e $S^{\prime}$ são dados por:

$$
d s^{2}(x)=g_{\alpha \beta}(x) d x^{\alpha} d x^{\beta} \quad \text { e } \quad d s^{\prime 2}\left(x^{\prime}\right)=g^{\prime} \mu v\left(x^{\prime}\right) d x^{\prime \mu} d x^{\prime v} \text {. }
$$

Como se tem dois referenciais onde são representados os mesmos pontos, o elemento de linha é um invariante, de modo que:

$$
d s^{\prime 2}\left(x^{\prime}\right)=d s^{2}(x)
$$

Substituindo-se as relações (2.13) na relação mencionada anteriormente e considerando-se (2.12), tem-se que os tensores métricos $g_{\alpha \beta}(x)$ e $g^{\prime} \mu \nu\left(x^{\prime}\right)$ se relacionam por:

$$
g^{\prime} \mu \nu\left(x^{\prime}\right) \frac{\partial x^{\prime} \mu}{\partial x^{\alpha}} \frac{\partial x^{\prime \nu}}{\partial x^{\beta}}=g_{\alpha \beta^{(x)}}
$$


Considerando-se o Jacobiano da transformação (2.11) e tomando-se $g$ e $g^{\prime}$, respectivamente, como os determinantes de $g_{\alpha \beta}(x)$ e $g^{\prime} \mu \nu\left(x^{\prime}\right)$, encontra-se:

$$
\left|J^{\prime}\right|=\left|\operatorname{det}\left[\frac{\partial x^{\prime \alpha}}{\partial x^{\mu}}\right]\right|=\sqrt{\frac{|g|}{\left|g^{\prime}\right|}} .
$$

Como foi exposto anteriormente, as transformações (ativas) entre pontos diferentes de um mesmo espaço são representadas pelas relações $\bar{x}^{\mu}=\bar{x}^{\mu}\left(x^{\alpha}\right)$, onde $x^{\mu}$ e $\bar{x}^{\mu}$ denotam, respectivamente, um ponto e seu transformado. Para as transformações entre sistemas de coordenadas (passivas) usa-se $x^{\prime \mu}=x^{\prime \mu}\left(x^{\alpha}\right)$, onde $x^{\alpha}$ e $x^{\prime \mu}$ representam as coordenadas de um mesmo ponto em dois sistemas de coordenadas diferentes. Pode-se verificar que existe uma conexão entre esses dois tipos de transformações [Be-84, Fu-62, Lo-77, Wo-89]. Uma das formas de se verificar isso [Be-84] pode ser feita igualando-se as coordenadas $\bar{x}$ de $S$ às coordenadas $x^{\prime}$ de $S^{\prime}$, ou seja:

$$
x^{\prime} \mu=\bar{x}^{\mu}
$$

Limitando-se ao caso das transformações conformes em espaços planos de $n$ dimensões, tem-se que os tensores métricos $\bar{g}_{\mu v}(\bar{x})$ e $g_{\mu \nu}(x)$ relativos a um referencial cartesiano S são dados por:

$$
\bar{g}_{\mu v}(\bar{x})=g_{\mu v}(x)=\eta_{\mu v}, \text { onde: } \eta_{\mu v}= \pm 1 \text {, se } \mu=v \text { e } \eta_{\mu v}=0, \text { se } \mu \neq v \text {. }
$$

Considerando-se a correlação (2.16) entre as transformações ativas e passivas, e utilizando-se as relações (2.10) e (2.14) para espaços planos, tem-se que, para uma transformação conforme das coordenadas cartesianas $x^{\mu}$ para as coordenadas curvilíneas $x^{\prime}{ }^{\mu}$, o tensor métrico $g^{\prime} \mu \nu\left(x^{\prime}\right)$ relativo a $S^{\prime}$ é dado por:

$$
g^{\prime} \mu v\left(x^{\prime}\right)=\frac{\eta_{\mu v}}{\sigma(x)} .
$$


Substituindo-se esse resultado na relação (2.14), tem-se que as transformações conformes de coordenadas em um espaço plano de $n$ dimensões são determinadas a partir da relação:

$$
\eta_{\mu v} \frac{\partial x^{\prime \mu}}{\partial x^{\alpha}} \frac{\partial x^{\prime \nu}}{\partial x^{\beta}}=\sigma(x) \eta_{\alpha \beta}
$$

A relação acima também pode ser dada em termos do Jacobiano $J^{\prime}$. Para isso, considera-se que esta relação pode ser escrita na forma:

$$
d x^{\prime \mu} d x_{\mu}^{\prime}=\sigma(x) d x^{\alpha} d x_{\alpha}
$$

Substituindo-se as relações $d x^{\prime} \mu^{\mu}=\frac{\partial x^{\prime \mu}}{\partial x^{\lambda}} d x^{\lambda}$ e $d x^{\prime}{ }_{\mu}=\frac{\partial x^{\prime} \mu}{\partial x_{\rho}} d x_{\rho}$ na relação acima, obtém-se a expressão:

$$
\left(\frac{\partial x^{\prime \mu}}{\partial x^{\lambda}}\right)\left(\frac{\partial x^{\prime}}{\partial x_{\rho}}\right) d x^{\lambda} d x_{\rho}=\sigma(x) d x^{\alpha} d x_{\alpha}
$$

Para se relacionar o fator de escala $\sigma(x)$ ao Jacobiano $J^{\prime}$, considera-se a relação (2.15) e os módulos dos determinantes de $g_{\alpha \beta}(x)$ e $g_{\mu \nu}^{\prime}\left(x^{\prime}\right)$ que, para transformações conformes em espaços planos de $n$ dimensões, são dados, respectivamente, por $|g|=1$ e $\left|g^{\prime}\right|=\sigma(x)^{-n}$. Dessa forma, tem-se que:

$$
\sigma(x)=\left|J^{\prime}\right|^{\frac{2}{n}}
$$

Substituindo-se o fator de escala acima na relação (2.18), obtém-se, finalmente: 


$$
\left(\frac{\partial x^{\prime \alpha}}{\partial x^{\lambda}}\right)\left(\frac{\partial x_{\alpha}^{\prime}}{\partial x_{\rho}}\right) d x^{\lambda} d x_{\rho}=\left|J^{\prime}\right|^{\frac{2}{n}} d x^{\mu} d x_{\mu} .
$$

De acordo com a relação acima, as transformações conformes de coordenadas num espaço plano de $n$ dimensões satisfazem a equação:

$$
\left(\frac{\partial x^{\prime \alpha}}{\partial x^{\lambda}}\right)\left(\frac{\partial x^{\prime} \alpha}{\partial x_{\rho}}\right)=\left|J^{\prime}\right|^{\frac{2}{n}} \delta_{\lambda}^{\rho} .
$$

A relação acima é equivalente à relação (2.17) e pode ser utilizada para a obtenção das transformações de coordenadas conformes num espaço plano de $n$ dimensões em termos do Jacobiano da transformação envolvendo as coordenadas covariantes e contravariantes.

Em particular, para o espaço-tempo plano, o tensor métrico é dado por:

$$
\eta_{\mu \nu}=1(\mu=v=1,2,3), \quad \eta_{\mu \nu}=-1(\mu=v=4) \text { e } \eta_{\mu \nu}=0(\mu \neq v)
$$

Um evento é dado, em coordenadas cartesianas ( $\operatorname{com} c=1$ ), pelo ponto:

$$
x \equiv\left(x^{1}, x^{2}, x^{3}, x^{4}\right)=(x, y, z, t)
$$

Como o espaço-tempo é quadridimensional $(n=4)$, tem-se que, neste caso, a relação (2.19) é dada por:

$$
\left(\frac{\partial x^{\prime \alpha}}{\partial x^{\lambda}}\right)\left(\frac{\partial x_{\alpha}^{\prime}}{\partial x_{\rho}}\right)=\sqrt{\left|J^{\prime}\right|} \delta_{\lambda}^{\rho} .
$$

A equação acima restringe a relação (2.19) para o espaço-tempo de Minkowski, e será útil, no capítulo $\mathrm{V}$ deste trabalho, para os cálculos utilizados a fim de se mostrar a invariância conforme das equações do campo eletromagnético. 


\subsection{Síntese da história das transformações conformes e suas aplicações.}

Historicamente, o estudo das transformações conformes teve a sua origem na cartografia, onde se desenvolveram as técnicas para o traçado de mapas da superfície da Terra [Do-05, Ka-08, Ro-11, Ca-09]. Tais mapas consistem, basicamente, na projeção de uma superfície esférica em outra superfície, em geral plana, cilíndrica ou cônica.

A projeção estereográfica [B1-00, Co-98, Ka-08, He-01, Ne-97] é um exemplo deste tipo de mapeamento, onde os pontos de uma superfície esférica são projetados nos pontos de um plano. Conhecida desde os tempos de Hiparco de Nicea (190-120 a. C.), esta projeção é descrita matematicamente na obra Planisphaerium, de Claudio Ptolomeu (90-168 d. C.) [Be-91, Lo-95]. A Fig. 2.3 representa, a partir do pólo sul, a projeção estereográfica dos trópicos e do equador num plano que corta a linha equatorial da Terra.

O estudo da projeção estereográfica foi bastante influenciado por sua aplicação na construção do astrolábio [Ne-49, No-74], um instrumento esférico ou em forma de círculo graduado, dotado de haste móvel, cuja invenção tem origem na Grécia antiga. O astrolábio era utilizado para se determinar a altura do Sol e das estrelas em relação ao horizonte e fazer medições de latitude. A Fig. 2.4 ilustra o astrolábio planisférico [Mu-13], composto por discos onde se representavam as linhas dos trópicos da Terra e as posições das estrelas. Neste instrumento, a projeção estereográfica era utilizada para representar a esfera celeste, uma vasta esfera onde os planetas, a lua e o sol giravam em torno da Terra, que era considerada sem rotação e fixa no centro da esfera.
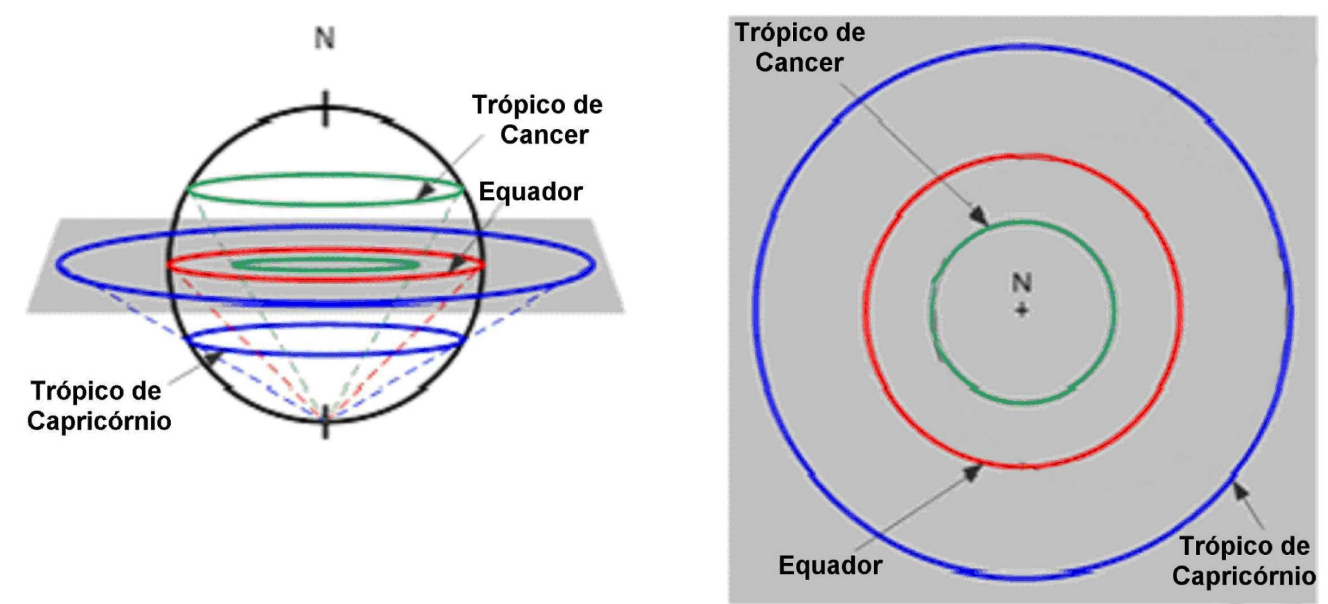

Fig. 2.3 - Representação da projeção estereográfica (a partir do pólo sul) de pontos na superfície terrestre em um plano que corta a linha equatorial. São mostradas as linhas dos trópicos e do equador. [As-13] 


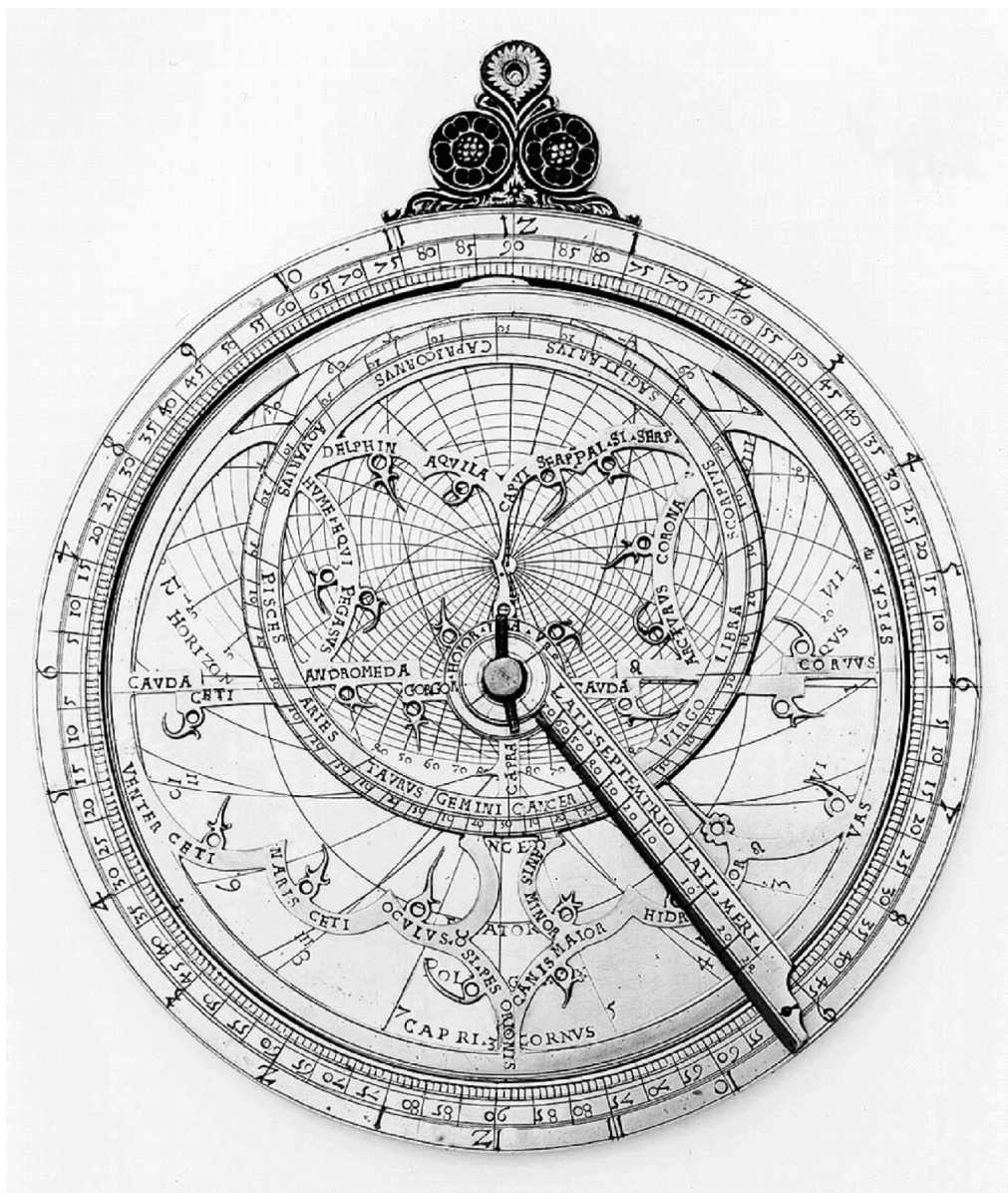

Fig. 2.4 - O astrolábio planisférico. [Mu-13]

Nos tempos de Ptolomeu, já se sabia que a projeção estereográfica tem a propriedade de transformar circunferências na superfície da esfera em circunferências ou retas no plano, mas esta propriedade foi provada somente na primeira metade do século IX pelo astrônomo e engenheiro Al-Farghani [Se-78], que viveu em Bagdá e no Cairo. Outra prova é devida ao matemático europeu Jordanus de Nemore por volta do ano de 1200 [Th-78].

No ano de 1593, Christopher Clavius (1537-1612) mostrou em seu livro Astrolabium [Cl-93] como determinar o ângulo formado pela intersecção de dois grandes círculos numa esfera através da medida do ângulo formado pelas imagens transformadas no plano. Embora o método de Clavius não tenha sido uma prova de que a projeção estereográfica é uma transformação conforme, era uma demonstração de que os ângulos eram preservados pela transformação. A prova geométrica de que a projeção estereográfica é conforme foi dada somente em 1696 por Edmond Halley (1656-1742) [Ha-09].

Os fundamentos da cartografia estão registrados nos trabalhos de Ptolomeu, que criou um mapa da Terra (Fig. 2.5) a partir da coleta de dados conhecidos em sua época. 
Durante a Idade Média, a Europa passou por um período de estagnação científica e os trabalhos de Ptolomeu passaram a ser estudados e desenvolvidos pelos árabes [Si-07].

Somente com o Renascimento, os trabalhos de Ptolomeu foram reintroduzidos na Europa pelos árabes e os mapas passaram a ser estudados para fins militares, comerciais e para a navegação. Neste período, percebeu-se que o mapa de Ptolomeu mostrava-se imperfeito, e o desenvolvimento da navegação marítima e a descoberta de novos continentes trouxeram a necessidade de um aperfeiçoamento dos mapas do globo terrestre. Citamos aqui, o mapa de Mercator, criado em 1569 por Gerhardus Mercator (1512-1594) e usado essencialmente para a navegação marítima [Sn-93]. Este mapa consiste na projeção de uma superfície esférica na superfície de um cilindro tangente ao equador da esfera, conforme mostrado na Fig. 2.6. O mapa de Mercator transforma os meridianos e paralelos em linhas retas paralelas entre si, preserva a forma, mas não o tamanho das figuras geométricas.

A demonstração, por meio do cálculo diferencial, de que a projeção estereográfica e a projeção de Mercator são transformações conformes, foi feita por Johann Heinrich Lambert (1728-1777) em 1772 [La-72]. Lambert propôs ainda outros mapeamentos conformes, entre eles a chamada projeção cônica conforme de Lambert [Sn-93], em que a superfície esférica é mapeada num cone que corta dois paralelos padronizados da esfera. Nesta projeção cônica, cujo centro de projeção está no centro da esfera, os paralelos são transformados em arcos de circunferência e os meridianos são transformados em retas não paralelas. A projeção cônica conforme de Lambert possui a vantagem de preservar as áreas originais de modo mais preciso que a projeção estereográfica e de Mercator. Na Fig. 2.7 tem-se um mapa-múndi obtido a partir da projeção de Lambert. 


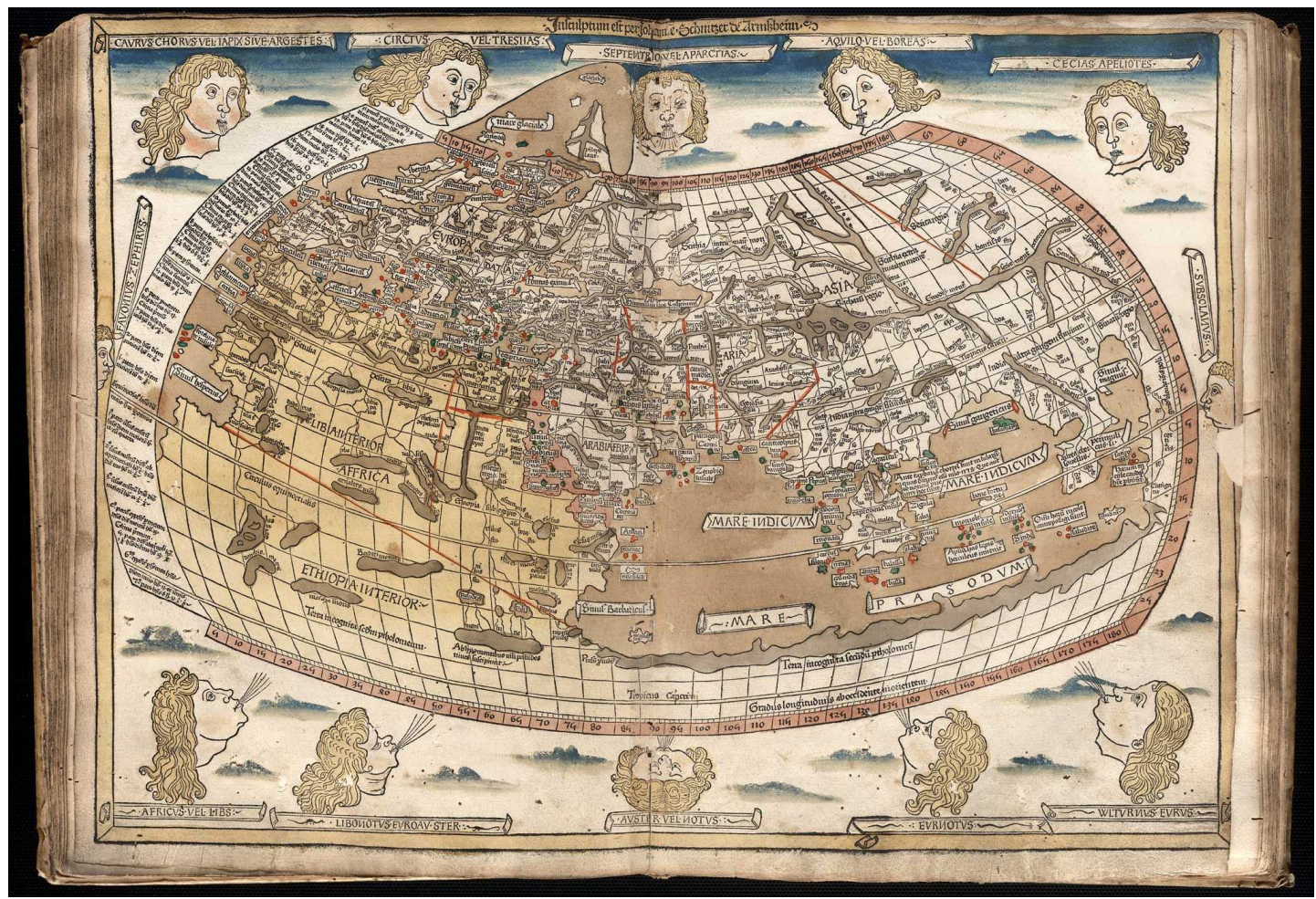

Fig. 2.5 - O mapa de Ptolomeu. [Bi-13]
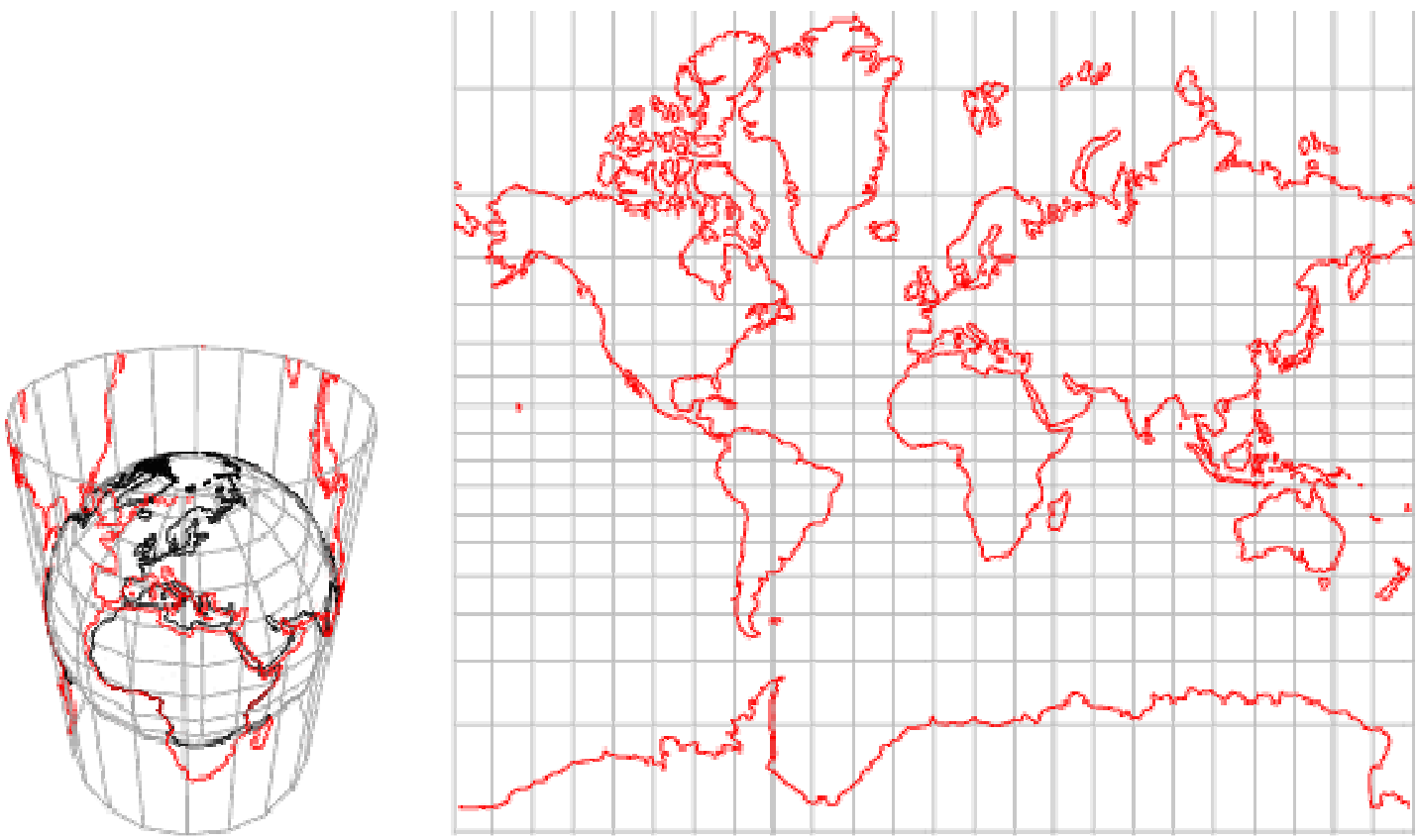

Fig. 2.6 - A projeção cilíndrica e o mapa de Mercator. Os pontos contidos na esfera são projetados na superfície cilíndrica tangente à esfera no equador, a partir do centro da esfera. [Ma-13, Ma-13a] 


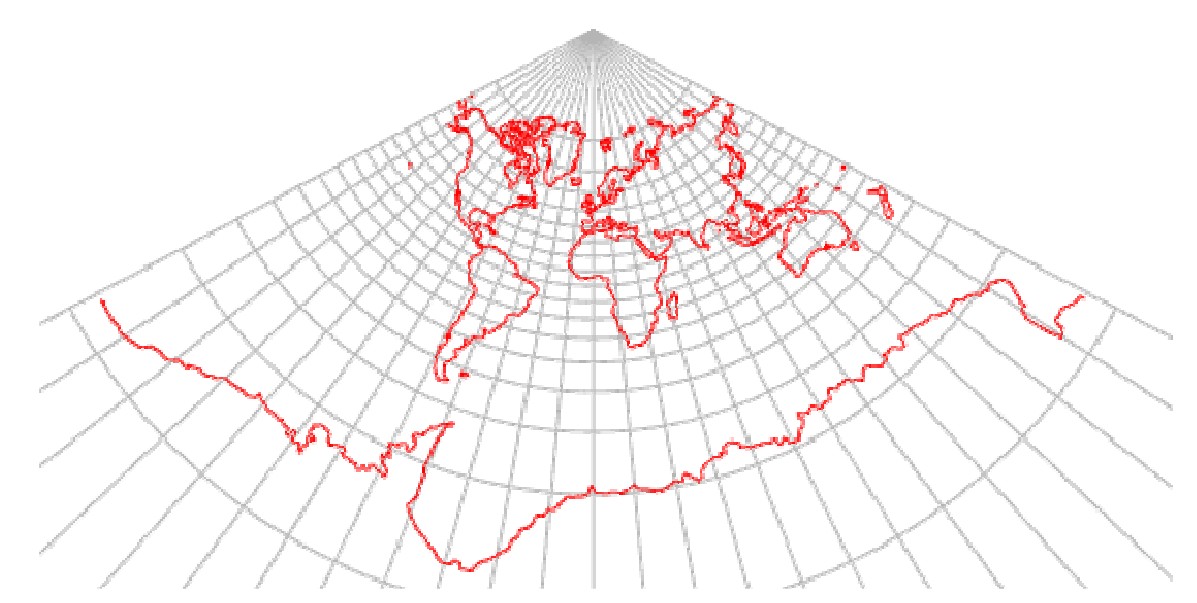

Fig. 2.7 - O mapa-múndi através da projeção cônica conforme de Lambert. [Ma-13b]

Destacam-se, ainda, neste período, os trabalhos de Leonhard Euler (1707-1783) [Sp-55] e de Joseph-Louis de Lagrange (1736-1813) [La-69], que, além dos seus trabalhos bastante conhecidos em matemática e em mecânica, também desenvolveram estudos em cartografia, devido à necessidade prática de mapas mais precisos demandada pelos cartógrafos. Os trabalhos de Euler, datados de 1777 para a Academia de São Petersburgo, abordavam o mapeamento conforme de uma superfície esférica num plano, onde, aparentemente pela primeira vez, era feita a parametrização das coordenadas do plano pela notação complexa $z=x+i y$. Euler forneceu as bases para a teoria geral das superfícies e demonstrou que é impossível mapear uma região de uma superfície esférica numa região plana sem que hajam distorções. Lagrange apresentou seus trabalhos sobre a construção de mapas geográficos para a Academia de Ciências de Berlim em 1779, generalizando os trabalhos de Lambert e Euler para todas as projeções que mapeiam círculos na esfera em círculos no plano.

Em 1822, Carl Friedrich Gauss (1777-1855) apresentou a solução geral do problema envolvendo o mapeamento de partes de uma determinada superfície sobre outra [Ga-28, La-15]. O problema foi proposto pela Sociedade Científica Real de Copenhagen e foi publicado posteriormente em 1825. Neste trabalho, Gauss demonstrou que o mapeamento de uma superfície regular qualquer num plano é localmente conforme. O termo "mapeamento conforme", no entanto, somente foi introduzido por Gauss em 1844.

Em 1827, Gauss publicou sua obra sobre a teoria geral das superfícies curvas [Mo-02], onde apresentava a demonstração de seu famoso teorema Egrégio (teorema notável). As ideias básicas para este trabalho foram obtidas a partir de um levantamento topográfico, 
feito por Gauss entre 1818 e 1825 para o rei de Hannover, para a confecção de um mapa de algumas regiões da Alemanha.

O teorema Egrégio afirma que a curvatura é um invariante para uma superfície bidimensional que for desenvolvida sobre outra isometricamente, ou seja, sem que se alterem as distâncias na superfície. A superfície de um cilindro, por exemplo, pode ser desenvolvida num plano, pois ambas as superfícies têm mesma curvatura (nula); já a superfície de uma esfera não pode ser desenvolvida sobre um plano sem que hajam deformações, pois essas superfícies têm curvaturas diferentes. Pelo teorema Egrégio, verifica-se que é impossível mapear uma região da superfície terrestre numa região plana sem que hajam distorções, provando que um mapeamento perfeito da Terra não é possível.

As ideias de Gauss foram continuadas e desenvolvidas por Georg Friedrich Bernhard Riemann (1826-1866) em seu trabalho de 1854 [Sa-88] para espaços $n$-dimensionais. Neste trabalho, feito a pedido do próprio Gauss para que Riemann obtivesse um cargo na Universidade de Göttingen, há a formulação das bases da geometria Riemanniana, que mais tarde seria utilizada por Albert Einstein (1879-1955) na elaboração da teoria da Relatividade Geral, publicada em 1916.

A contribuição essencial de Riemann para o estudo das transformações conformes no plano está em sua tese de doutorado de 1851 sobre a teoria geral das funções complexas [La-99, Ri-90]. Nesta tese, há a formulação do teorema do mapeamento conforme de Riemann, o qual estabelece que toda região simplesmente conexa do plano complexo pode ser mapeada por transformações conformes no interior do círculo unitário. Riemann esclareceu as condições de diferenciabilidade de uma função complexa, até então estudadas por Augustin Louis Cauchy (1789-1857), estabelecendo as chamadas condições de Cauchy-Riemann, e verificou que uma função analítica define uma transformação conforme no plano complexo. Riemann verificou ainda que as condições de Cauchy-Riemann implicam na equação de Laplace em duas dimensões. Estas propriedades das funções complexas são utilizadas para a resolução de problemas de contorno por transformações conformes em duas dimensões, onde um problema com uma geometria complicada pode ser tratado em uma geometria mais simples através do mapeamento conforme das variáveis complexas.

Riemann estabeleceu também uma relação entre o plano complexo e a projeção estereográfica utilizada em cartografia, definindo a chamada esfera de Riemann [Bu-88, Ne-97]. Nesta projeção, uma esfera de diâmetro unitário tangencia o plano complexo na origem. Os pontos da superfície esférica são transformados nos pontos do plano complexo através de uma reta que intercepta estes pontos e o pólo norte da esfera, conforme 
representado na Fig. 2.8. Por meio desta projeção, todos os pontos da esfera são representados no chamado plano complexo estendido, isto é, o plano complexo com a adição de um ponto no infinito, correspondente ao pólo norte da esfera. O conceito de ponto no infinito estabelecido por Riemann está relacionado diretamente ao estudo das singularidades das funções analíticas no plano complexo.

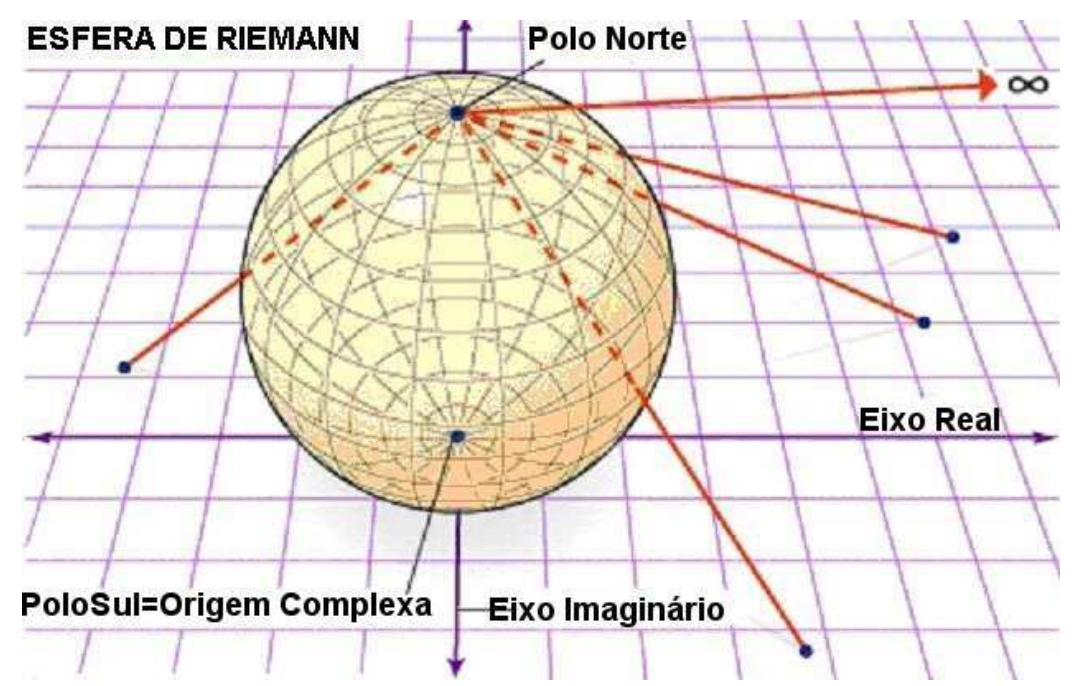

Fig. 2.8 - A esfera de Riemann e a projeção estereográfica no plano complexo. [Br-13]

Paralelamente ao desenvolvimento dos estudos sobre a geometria diferencial e as funções complexas, outros pesquisadores estudavam as transformações geométricas entre uma superfície esférica e um plano ou entre uma circunferência e uma reta. No período compreendido entre 1820 e 1850, vários pesquisadores que estudavam essas transformações descobriram de forma independente a transformação geométrica de inversão [Ba-74, Co-67, Co-69, Co-70, Em-14, Em-15, Em-29, Ne-97, Pa-33]. Por meio desta transformação num plano, os pontos internos a uma dada circunferência $C$ são transformados nos pontos externos, e vice-versa, de acordo com a Fig. 2.9, onde se considera uma circunferência de raio $a$ e centro $O$. A inversão transforma o ponto $P$ (interno à circunferência) no ponto $P^{\prime}$ (externo à circunferência), e vice-versa, de acordo com a relação $\overline{O P} \cdot \overline{O P}^{\prime}=a^{2}$.

Dentre os vários trabalhos sobre a inversão, citamos os de Germinal Pierre Dandelin (1794-1847), de 1825; de Lambert Adolphe Jacques Quetelet (1796-1874), também de 1825; Julius Plücker (1801-1868), de 1831; J. L Magnus (1790-1861), de 1832; Giusto Bellavitis (1803-1880), de 1836; e de August Ferdinand Möbius (1790-1868), de 1853. Considera-se que Jakob Steiner (1796-1863) tenha sido o primeiro a descobrir a transformação de inversão 
por volta do ano de 1824. No entanto, seus trabalhos sobre a inversão somente foram publicados postumamente, em 1913 [Em-29].

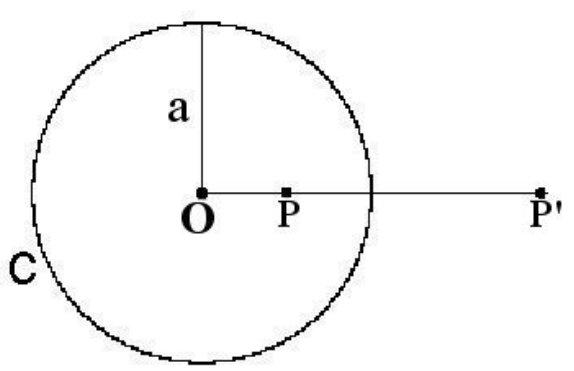

Fig. 2.9 - A transformação geométrica de inversão em duas dimensões: dada uma circunferência $\mathrm{C}$, de raio $a$ e centro $\mathrm{O}$, a inversão transforma o ponto $\mathrm{P}$ (interno à circunferência) no ponto $\mathrm{P}^{\prime}$ (externo à circunferência), e vice-versa, de acordo com a relação $\overline{O P} \cdot \overline{O P}^{\prime}=a^{2}$.

No ano de 1845, William Thomson (Lord Kelvin) (1824-1907) descobriu a transformação de inversão em três dimensões quando trabalhava na resolução de problemas eletrostáticos envolvendo esferas carregadas [Th-72]. Entre os anos de 1845 e 1847, Kelvin enviou algumas cartas a Joseph Liouville (1809-1882), onde discutia a resolução destes problemas pelo método encontrado [Th-45, Th-47, Th-72]. Kelvin descobrira que as soluções dos problemas eletrostáticos em três dimensões estavam relacionadas à invariância da equação de Laplace pela transformação de inversão. Por meio desta descoberta, Kelvin criou o chamado método das imagens para a resolução de problemas eletrostáticos. A Fig. 2.10 representa a aplicação do método das imagens para o problema do cálculo do potencial provocado por uma esfera condutora de raio $a$, colocada a um potencial nulo $V=0$, e uma carga puntiforme $Q_{0}$, localizada numa posição $r_{0}$ em relação ao centro da esfera. Este problema é equivalente ao cálculo do potencial provocado pela carga $Q_{0}$, localizada em $r_{0}$, e uma carga imagem $Q_{a}$, localizada na posição $r_{a}$, onde as posições $r_{0}$ e $r_{a}$ são dadas pela transformação de inversão em relação ao raio a da esfera, isto é, $r_{0} \cdot r_{a}=a^{2}$. O trabalho de Kelvin foi apreciado por James Clerk Maxwell (1831-1879) e o capítulo XI de seu tratado sobre eletricidade e magnetismo [Ma-73] é dedicado aos métodos de resolução de Kelvin pela transformação de inversão.

A descoberta de Kelvin motivou Liouville a formular um teorema sobre as transformações conformes no espaço de três dimensões. Este teorema [B1-00, Ku-07, Li-50, Mo-50, Ph-69] estabelece que as transformações conformes, no espaço euclidiano 
tridimensional, são restritas a translações, rotações, dilatações, inversões e composições destas transformações, diferentemente do que ocorre no plano, onde o número de transformações conformes é infinito. O teorema de Liouville desencadeou uma série de outros trabalhos sobre as transformações conformes em três dimensões durante o período entre 1850 e 1900, inclusive um artigo Maxwell, datado de 1872 [Ma-72].

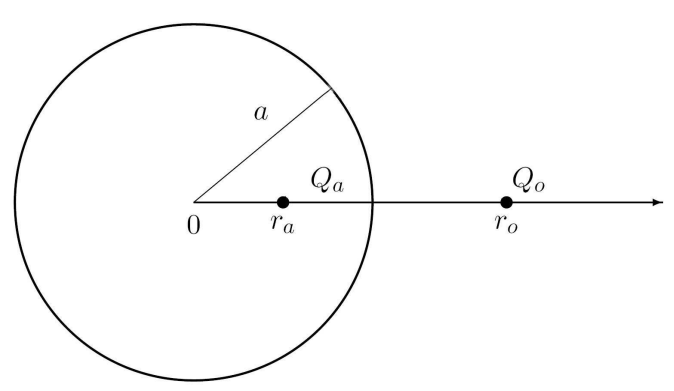

Fig. 2.10 - O método das imagens envolve a transformação de inversão e é utilizado na resolução de problemas, como por exemplo, o problema clássico da eletrostática envolvendo o cálculo do potencial no espaço de uma esfera condutora (raio $a$ e $V=0$ ) na presença de uma carga puntiforme $Q_{0}\left(\mathrm{em} r_{0}\right.$ ). O problema é equivalente ao potencial de uma carga $Q_{0}$ (em $r_{0}$ ) e uma carga imagem $Q_{a}\left(\right.$ em $\left.r_{a}\right)$ com $r_{0} \cdot r_{a}=a^{2}$.

As transformações conformes em espaços planos de $n$ dimensões $(n>2)$ euclidianos e não euclidianos foram determinadas por Sophus Lie (1842-1899) [Li-71, Li-72, Li-86], em 1871, que ampliou o resultado de Liouville, provando que nestes espaços as transformações conformes também são restritas.

Enquanto as transformações conformes em espaços planos $n$-dimensionais eram estabelecidas nos anos de 1870, no campo da Física, a obra fundamental de Maxwell sobre a teoria eletromagnética era publicada em 1873 [Ma-73]. Nesta obra, encontra-se a descrição da teoria que unifica a eletricidade, o magnetismo e a óptica, sintetizada na forma das hoje chamadas equações de Maxwell.

Considerando as implicações físicas da teoria eletromagnética de Maxwell, Einstein formulou, em 1905, a teoria da relatividade restrita. Nesta formulação, Einstein postulou que as equações da eletrodinâmica mantêm a sua forma em relação a qualquer referencial inercial. Como as transformações de Galileu não mantêm a forma das equações de Maxwell, elas tiveram que ser descartadas e em seu lugar foram colocadas as transformações de Lorentz, que deixam as equações da eletrodinâmica invariantes. Essa teoria teve implicações importantes em toda a Física e foi, como outras teorias importantes da Física, uma teoria 
unificadora, principalmente unificando o espaço e o tempo, pois antes da relatividade restrita o tempo era considerado absoluto.

Como conseqüência desta formulação, Hermann Minkowski (1864-1909) propôs em 1908 que o espaço onde ocorrem os eventos físicos é um espaço-tempo quadridimensional com uma métrica definida, hoje chamado de espaço-tempo de Minkowski [Pe-52]. As transformações de Lorentz passaram então a ser vistas como rotações no espaço-tempo de Minkowski. Estas rotações possuem a propriedade de serem transformações conformes no espaço-tempo.

No mesmo ano de 1908, Harry Bateman (1882-1946) publicou um artigo sobre as transformações conformes no espaço de quatro dimensões [Ba-09, Wa-92]. O trabalho de Bateman, no entanto, não se relacionava com a proposta de Minkowski e nem citava os artigos de Einstein, Lorentz e Poincaré sobre a relatividade. O artigo era motivado pela determinação das transformações conformes que deixam a equação de onda eletromagnética invariante, ou seja, foi uma generalização do trabalho de Kelvin sobre a invariância da equação de Laplace pela transformação de inversão no espaço tridimensional. Bateman considerou a equação de onda como uma generalização da equação de Laplace, introduzindo as variáveis $w=i c t$ e $r^{2}=x^{2}+y^{2}+z^{2}+w^{2}$ para deixá-la simétrica. $\mathrm{O}$ objetivo de Bateman era demonstrar que as leis de refração e reflexão da óptica geométrica são invariantes pela transformação de inversão num espaço de quatro dimensões $(x, y, z, i c t)$.

Pouco tempo depois, em 1910, Bateman e seu colega Ebenezer Cunningham (18811977) publicaram dois artigos onde a invariância conforme das equações do eletromagnetismo era demonstrada [Ba-10, $\mathrm{Cu}-10, \mathrm{Wa}-92]$.

Cunningham e Bateman tiveram suas formações na Universidade de Cambridge, onde obtiveram a distinção Senior Wrangler, a maior distinção atribuída a matemáticos nessa Universidade. Também receberam o prêmio Smith's Prize respectivamente de 1904 e 1905. Esse prêmio era concedido anualmente pela mesma universidade a estudantes que faziam pesquisa em física teórica ou em matemática aplicada. Ambos tinham um grande interesse pela área da física-matemática. O interesse em comum pelas soluções de equações diferenciais e integrais, e o conhecimento, por parte de Cunningham, do artigo de Einstein de 1905, fizeram com que Bateman e Cunningham trabalhassem juntos à procura das transformações gerais que deixassem as equações de Maxwell invariantes. Além disso, Bateman e Cunningham conheciam as transformações conformes e estavam interessados na 
aplicação física da técnica de inversão até então utilizada por Kelvin em problemas eletrostáticos.

Os artigos de Bateman e Cunningham de 1910 demonstravam que as equações de Maxwell são invariantes não somente pelas transformações de Poincaré, mas também pelo grupo mais geral de transformações conformes no espaço-tempo. Enquanto o artigo de Bateman aborda, de um modo geral, as transformações conformes no espaço-tempo, o artigo de Cunningham considera somente a invariância pela transformação conforme de inversão. No artigo de Bateman há uma menção sobre as transformações mais gerais entre referenciais que deixam as equações de Maxwell invariantes e uma referência à transformação de coordenadas entre referenciais com aceleração constante, abordada por Einstein em 1907 [Ei-07]. Já no artigo de Cunningham há a formulação de uma extensão do princípio da relatividade restrita para referenciais com aceleração constante, baseada na invariância das equações do eletromagnetismo pela transformação de inversão no espaço-tempo. A partir destas considerações, vários pesquisadores utilizaram uma transformação conforme específica, formada por uma translação, uma inversão e outra translação no espaço-tempo, denominada transformação conforme especial, como uma transformação entre um referencial inercial e outro com aceleração constante (no apêndice A faz-se uma discussão sobre esta associação entre as transformações conformes especiais e o movimento uniformemente acelerado). Esta interpretação para a transformação conforme especial foi considerada em vários artigos sobre a invariância conforme em Física a partir da terceira década do século XX até o início da década de 1960, quando houve uma reinterpretação física para esta transformação, considerada como uma transformação de escala dependente das coordenadas do espaço-tempo [Ka-66].

Embora os artigos de Bateman e de Cunningham de 1910 abordassem a invariância conforme das leis do eletromagnetismo, as leis de conservação associadas a esta invariância foram discutidas posteriormente.

Em 1915, Emmy Noether (1882-1935) provou o famoso e importante teorema que leva o seu nome, publicado em 1918 [No-18, Ta-71], segundo o qual se pode relacionar uma lei de conservação a uma simetria contínua. Em 1920, o matemático Felix Klein (1849-1925) solicitou a Erich Bessel-Hagen (1898-1946) que aplicasse o teorema de Noether para determinar as leis de conservação resultantes da invariância conforme das equações de Maxwell [A1-06, Be-21, Pl-74, Ro-08].

O artigo de Bessel-Hagen, publicado em 1921, foi uma das primeiras aplicações do teorema de Noether. Nele, Bessel-Hagen demonstrou a conservação da energia, dos 
momentos linear e angular e a lei de conservação relativística para o centro de energia do campo eletromagnético. Mostrou também que a invariância conforme das equações do eletromagnetismo implicava na existência de uma lei de conservação associada à simetria de dilatação no espaço-tempo, também denominada simetria de escala.

O interesse pelas transformações de escala em física, já conhecidas e aplicadas, por exemplo, em mecânica clássica, foi renovado a partir da década de 1960, com a possibilidade de aplicação da invariância de escala em processos envolvendo o espalhamento profundamente inelástico elétron-próton a altas energias [Ka-08].

As transformações conformes, utilizadas em Física inicialmente para a resolução de problemas eletrostáticos, ganharam um novo status com a publicação dos artigos de Bateman e Cunningham. A descoberta da invariância conforme das equações de Maxwell marcou o início das aplicações e discussões sobre o significado físico das transformações conformes em teorias de campos. Atualmente, a invariância conforme é aplicada em várias áreas da Física, como mecânica estatística e teoria das cordas, por exemplo [B1-09].

Este relato resumido da história das transformações conformes mostra como questões práticas, ligadas, por exemplo, ao comércio e às grandes navegações levaram diretamente a formulações teóricas importantes e envolveram nomes como Euler, Lagrange, Gauss, entre outros. 


\section{CAPÍTULO III}

\section{A ELETRODINÂMICA CLÁSSICA E O TEOREMA DE NOETHER.}

Neste capítulo, faz-se inicialmente uma revisão da eletrodinâmica covariante e do formalismo Lagrangiano para meios contínuos aplicado ao campo eletromagnético. A seguir, discute-se o teorema de Noether para campos e sua aplicação na eletrodinâmica.

\subsection{Alguns resultados da eletrodinâmica covariante.}

Os fenômenos eletromagnéticos são descritos pelas quatro equações de Maxwell [Fr-05, Gr-99, He-95, Ja-99], que relacionam os campos elétrico $(\vec{E})$ e magnético $(\vec{B})$ com suas fontes $(\rho$ e $\vec{J}$ ). Em unidades de Heavyside-Lorentz (com $c=1$ ) [Gr-96] essas equações são dadas por:

$$
\begin{aligned}
\vec{\nabla} \cdot \vec{E}=\rho \quad(3.1), & \vec{\nabla} \cdot \vec{B}=0 \\
\vec{\nabla} \times \vec{E}+\frac{\partial \vec{B}}{\partial t}=0 & \quad(3.3), \quad \vec{\nabla} \times \vec{B}-\frac{\partial \vec{E}}{\partial t}=\vec{j}
\end{aligned}
$$

As relações (3.2) e (3.3) são as equações homogêneas e as relações (3.1) e (3.4) são conhecidas como as equações não homogêneas de Maxwell. A equação (3.1) é denominada lei de Gauss para $\vec{E}$, cuja base experimental é a lei de Coulomb; a equação (3.2) representa a mesma lei para $\vec{B}$ e descreve a ausência de monopolos magnéticos; a equação (3.3) é a lei de Faraday e, finalmente, a equação (3.4) é conhecida nos textos como a Lei de Ampére (com o termo da corrente de deslocamento de Maxwell). Essas duas últimas também nasceram de bases experimentais.

A equação (3.2) implica que $\vec{B}$ pode ser expresso como o rotacional de um campo vetorial, o potencial vetor $\vec{A}$, na forma:

$$
\vec{B}=\vec{\nabla} \times \vec{A} .
$$


A relação acima, substituída em (3.3), permite que o campo elétrico $\vec{E}$ possa ser escrito em termos dos potenciais vetor $\vec{A}$ e escalar $\Phi$, ou seja:

$$
\vec{E}=-\vec{\nabla} \Phi-\frac{\partial \vec{A}}{\partial t}
$$

Deve-se observar, porém, que os potenciais eletromagnéticos não são determinados de modo único. Tomando-se um novo $\operatorname{par}\left(\vec{A}^{\prime}, \Phi^{\prime}\right)$, relacionado com o par original $(\vec{A}, \Phi)$ de tal forma que:

$$
\overrightarrow{A^{\prime}}=\vec{A}+\vec{\nabla} \psi \quad \text { e } \quad \Phi^{\prime}=\Phi-\frac{\partial \psi}{\partial t}
$$

verifica-se que a substituição das relações acima em (3.5) e (3.6) leva aos mesmos campos $\vec{E}$ e $\vec{B}$ fisicamente relevantes. Nestas relações, $\psi(x, y, z, t)$ é uma função escalar arbitrária do espaço e do tempo. Considerando-se essa liberdade na escolha de $\vec{A}$ e $\Phi$, pode-se supor que eles satisfazem a condição de Lorentz:

$$
\vec{\nabla} \cdot \vec{A}+\frac{\partial \Phi}{\partial t}=0
$$

Neste caso, $\vec{A}$ e $\Phi$ satisfazem equações de onda não homogêneas e desacopladas, que são equivalentes às equações de Maxwell, dadas por:

$$
\nabla^{2} \vec{A}-\frac{\partial^{2} \vec{A}}{\partial t^{2}}=-\vec{j} \quad \text { e } \quad \nabla^{2} \Phi-\frac{\partial^{2} \Phi}{\partial t^{2}}=-\rho
$$

Essas relações constituem um conjunto de equações desacopladas com soluções muitas vezes mais simples de serem obtidas que as das equações (3.1-3.4).

Definindo-se, no espaço-tempo de Minkowski, o quadrivetor:

$$
A^{\mu}=\left(A^{1}, A^{2}, A^{3}, A^{4}\right)=(\vec{A}, \Phi),
$$


tem-se que o campo elétrico $\vec{E}$ e o campo magnético $\vec{B}$ são dados, respectivamente, pelas relações:

$$
\vec{E}=-\left(\frac{\partial A^{4}}{\partial x^{1}}, \frac{\partial A^{4}}{\partial x^{2}}, \frac{\partial A^{4}}{\partial x^{3}}\right)-\frac{\partial}{\partial x^{4}}\left(A^{1}, A^{2}, A^{3}\right)
$$

$\mathrm{e}$

$$
\vec{B}=\vec{\nabla} \times\left(A^{l}, A^{2}, A^{3}\right)
$$

Usando-se $A^{\mu}$, a condição de Lorentz pode ser escrita na forma:

$$
\frac{\partial A^{\mu}}{\partial x^{\mu}}=0
$$

que é um invariante por transformações de Lorentz, ou seja, tem a mesma forma em qualquer sistema de referência inercial.

Outro quadrivetor importante na eletrodinâmica relativística é o quadrivetor densidade de corrente: $j^{\mu}=\left(j^{1}, j^{2}, j^{3}, j^{4}\right)=(\vec{j}, \rho)$.

Utilizando-se os quadrivetores $A^{\mu}$ e $j^{\mu}$, tem-se que as equações de onda (3.7) podem ser expressas em uma única relação quadridimensional:

$$
\left(\nabla^{2}-\frac{\partial}{\partial x^{4}} \frac{\partial}{\partial x^{4}}\right) A^{\mu}=\square A^{\mu}=-j^{\mu}
$$

onde as partes espacial e temporal correspondem, respectivamente, às equações de onda para $\vec{A}$ e $\Phi$, e $\square$ é operador de d'Alembert.

$\vec{A}$ e $\Phi$ são componentes de um quadrivetor, mas o mesmo não ocorre com os campos $\vec{E}$ e $\vec{B}$. Verifica-se que as seis componentes dos campos $\vec{E}$ e $\vec{B}$ são elementos de um tensor de segunda ordem, o tensor eletromagnético $F^{\mu v}$, que é dado por:

$$
F^{\mu v}=\frac{\partial A^{v}}{\partial x_{\mu}}-\frac{\partial A^{\mu}}{\partial x_{v}}=\partial^{\mu} A^{v}-\partial^{v} A^{\mu}
$$


onde se utilizou a notação: $\partial^{\mu} \equiv \frac{\partial}{\partial x_{\mu}}$.

$F^{\mu v}$ é um tensor anti-simétrico e suas formas contravariante e covariante são dadas, respectivamente, por:

$$
F^{\mu v}=\left(\begin{array}{cccc}
0 & B_{3} & -B_{2} & -E_{1} \\
-B_{3} & 0 & B_{1} & -E_{2} \\
B_{2} & -B_{1} & 0 & -E_{3} \\
E_{1} & E_{2} & E_{3} & 0
\end{array}\right) \quad \text { e } \quad F_{\mu v}=\eta_{\mu \alpha} F^{\alpha \beta} \eta_{\beta v}=\left(\begin{array}{cccc}
0 & B_{3} & -B_{2} & E_{1} \\
-B_{3} & 0 & B_{1} & E_{2} \\
B_{2} & -B_{1} & 0 & E_{3} \\
-E_{1} & -E_{2} & -E_{3} & 0
\end{array}\right) \text {, }
$$

onde $\eta_{\mu \alpha}=(1,1,1,-1), F_{\mu v}=\frac{\partial A_{v}}{\partial x^{\mu}}-\frac{\partial A_{\mu}}{\partial x^{\nu}}=\partial_{\mu} A_{v}-\partial_{\nu} A_{\mu}$ e $\partial_{\mu} \equiv \frac{\partial}{\partial x^{\mu}}$.

Deste modo, as componentes dos campos $\vec{E}$ e $\vec{B}$ podem ser expressas em relação a dois referenciais inerciais no espaço-tempo, $S$ e $S^{\prime}$, por meio da transformação de coordenadas do tensor eletromagnético $F^{\mu v}$, dada por:

$$
F^{\prime \mu \nu}=\frac{\partial x^{\prime \mu}}{\partial x^{\alpha}} \frac{\partial x^{\prime \nu}}{\partial x^{\beta}} F^{\alpha \beta}
$$

Por meio do tensor $F^{\mu \nu}$, as equações de Maxwell homogêneas (3.2) e (3.3) são dadas por:

$$
\frac{\partial F_{\rho \mu}}{\partial x^{v}}+\frac{\partial F_{\mu v}}{\partial x^{\rho}}+\frac{\partial F_{v \rho}}{\partial x^{\mu}}=0 .
$$

Em termos do tensor de Levi-Civita $\varepsilon^{\mu \nu \rho \sigma}$, a equação acima fica na forma:

$$
\varepsilon^{\mu v \rho \sigma} \frac{\partial F_{v \rho}}{\partial x^{\sigma}}=0
$$

onde $\varepsilon^{\mu v \rho \sigma}$ é definido por: 
$\varepsilon^{\mu v \rho \sigma}=+1$, para $\mu=1, v=2, \rho=3, \sigma=4$ e suas permutações pares;

$\varepsilon^{\mu v \rho \sigma}=-1$, para permutações ímpares;

$\varepsilon^{\mu v \rho \sigma}=0$, para combinações em que dois ou mais índices sejam iguais.

As equações não homogêneas, (3.1) e (3.4), são dadas em termos de $F^{\mu v}$ por:

$$
\frac{\partial F^{\mu v}}{\partial x^{v}}=j^{\mu}
$$

A seguir, discute-se sucintamente a obtenção da equação de Euler-Lagrange para meios contínuos, que servirá de base teórica para a formulação Lagrangiana para o campo eletromagnético e para aplicação do Teorema de Noether.

\subsection{A formulação Lagrangiana para campos.}

Considera-se, inicialmente e de forma sucinta, a formulação Lagrangiana para sistemas discretos e, a seguir, a passagem desta para sistemas com um número infinito de graus de liberdade [Go-80, Le-07, Jo-98], como é o caso do campo eletromagnético.

Na mecânica Lagrangiana de partículas [Th-04], um sistema é descrito pelas coordenadas e velocidades generalizadas, $q_{i}(t)$ e $\dot{q}_{i}(t)$, respectivamente. Para um sistema com um número finito de graus de liberdade, $(i=1,2, \ldots N)$, as equações de movimento do sistema podem ser obtidas a partir da função Lagrangiana do sistema, $L\left[q_{i}(t), \dot{q}_{i}(t), t\right]$, que depende de $q_{i}, \dot{q}_{i}$ e, em certas situações, pode também depender explicitamente do tempo $t$. A função $L$ é dada por $L=T-U$, onde $T$ é a energia cinética, que pode ser escrita como:

$$
T=\frac{1}{2} \sum_{i} m_{i}\left(\sum_{j} \frac{\partial x_{i}}{\partial q_{j}} \dot{q}_{j}+\frac{\partial x_{i}}{\partial t}\right)^{2}
$$

e $U$ é a energia potencial, $U=U\left(q_{i}, \dot{q}_{i}\right)$, em geral não dependente do tempo. 
Define-se a ação $S$ pela integral: $S=\int_{t_{l}}^{t_{2}} L\left[q_{i}(t), \dot{q}_{i}(t), t\right] d t$. De acordo com o princípio de Hamilton, o movimento real do sistema é aquele em que a ação é um extremo, ou seja:

$$
\delta S=\delta \int_{t_{1}}^{t_{2}} L\left[q_{i}(t), \dot{q}_{i}(t), t\right] d t=0
$$

Essa condição leva às equações diferenciais de Euler-Lagrange:

$$
\frac{\partial L}{\partial q_{i}}-\frac{d}{d t} \frac{\partial L}{\partial \dot{q}_{i}}=0, i=1,2, \ldots N
$$

São $N$ equações diferenciais ordinárias de segunda ordem que, juntamente com as $2 N$ condições iniciais, permitem a determinação da trajetória do sistema, ou seja, $q_{i}(t)$.

As equações (3.10) são válidas para sistemas com um número finito de graus de liberdade. Quando se consideram sistemas contínuos, ou seja, com um número infinito de graus de liberdade, utiliza-se o formalismo Lagrangiano para sistemas contínuos.

Um exemplo simples e muito usado na literatura para a introdução à abordagem lagrangiana para meios contínuos é o caso de uma corda vibrante estendida ao longo do eixo $x$, com movimento no plano $x y$. Para esse sistema, a Lagrangiana é dada por:

$$
L=\int \frac{1}{2}\left[\mu\left(\frac{\partial \varphi}{\partial t}\right)^{2}-Y\left(\frac{\partial \varphi}{\partial x}\right)^{2}\right] d x=\int \mathcal{L} d x
$$

onde $\varphi=\varphi(x, t)$ representa a deformação da corda no eixo $y$, em relação à sua configuração de equilíbrio, $\mu$ é a densidade linear de massa da corda e $Y$ é o módulo de Young, definido como a razão entre a força $F$ ao longo da corda e a deformação da corda por unidade de comprimento. A grandeza:

$$
\mathcal{L}=\mathcal{L}\left(\frac{\partial \varphi}{\partial x}, \frac{\partial \varphi}{\partial t}\right)=\frac{1}{2}\left[\mu\left(\frac{\partial \varphi}{\partial t}\right)^{2}-Y\left(\frac{\partial \varphi}{\partial x}\right)^{2}\right]
$$


é chamada de densidade da Lagrangiana, que numa situação mais geral pode também depender de $\varphi$.

A aplicação do princípio de Hamilton a esse sistema, ou seja:

$$
\delta \int L d t=\delta \iint \mathcal{L}\left(\frac{\partial \varphi(x, t)}{\partial x}, \frac{\partial \varphi(x, t)}{\partial t}\right) d x d t=0
$$

leva à equação de Euler-Lagrange para $\varphi(x, t)$ :

$$
\frac{\partial \mathcal{L}}{\partial \varphi}-\frac{d}{d x}\left[\frac{\partial \mathcal{L}}{\partial\left(\frac{\partial \varphi}{\partial x}\right)}\right]-\frac{d}{d t}\left[\frac{\partial \mathcal{L}}{\partial\left(\frac{\partial \varphi}{\partial t}\right)}\right]=0
$$

Inserindo-se $\mathcal{L}$, dada por (3.11), nessa relação, tem-se a equação de onda para $\varphi(x, t):$

$$
\frac{\partial^{2} \varphi}{\partial x^{2}}=\frac{1}{v^{2}} \frac{\partial^{2} \varphi}{\partial t^{2}}
$$

onde $v=\sqrt{\frac{Y}{\mu}}$ é a velocidade de propagação de ondas elásticas longitudinais na corda.

O formalismo acima, desenvolvido para um sistema contínuo unidimensional, pode ser estendido para um sistema de $N$ campos $\left.\varphi \rho^{\left(x^{\mu}\right.}\right),(\rho=1, \ldots N)$, dependentes do espaço e do tempo, ou seja, de $x^{\mu},(\mu=1, \ldots 4)$, com: $x^{1}=x, x^{2}=y, x^{3}=z$ e $x^{4}=t$.

Nessa extensão, parte-se da densidade da Lagrangiana dependente dos campos $\varphi_{\rho}$ e suas derivadas $\partial_{\mu} \varphi_{\rho} \equiv \frac{\partial \varphi_{\rho}}{\partial x^{\mu}}$, ou seja, de:

$$
\mathcal{L}=\mathcal{L}\left(\varphi_{\rho}, \frac{\partial \varphi_{\rho}}{\partial x^{\mu}}\right)=\mathcal{L}\left(\varphi_{\rho}, \partial_{\mu} \varphi_{\rho}\right)
$$


A ação $S$, neste caso, é dada por:

$$
S=\int \mathcal{L}\left(\varphi_{\rho}, \partial_{\mu} \varphi_{\rho}\right) d x d y d z d t=\int \mathcal{L}\left(\varphi_{\rho}, \partial_{\mu} \varphi_{\rho}\right) d^{4} x
$$

e o princípio de Hamilton implica que:

$$
\delta S=\int \delta \mathcal{L}\left(\varphi_{\rho}, \partial_{\mu} \varphi_{\rho}\right) d^{4} x=0
$$

A variação local do campo é definida através da operação $\delta$ pela seguinte relação:

$$
\delta \varphi_{\rho}(x)=\varphi_{\rho}^{\prime}(x)-\varphi_{\rho}(x)
$$

e representa a mudança de forma do campo num ponto particular $x$.

Utilizando-se a variação acima para $\partial_{\mu} \varphi_{\rho}(x)$, tem-se que a variação $\delta$ e o operador de diferenciação $\partial_{\mu}$ comutam entre si, ou seja:

$$
\delta\left[\partial_{\mu} \varphi_{\rho}(x)\right]=\partial_{\mu}\left[\varphi_{\rho}^{\prime}(x)-\varphi_{\rho}(x)\right]=\partial_{\mu}\left[\delta \varphi_{\rho}(x)\right]
$$

A variação da densidade da Lagrangiana é dada por:

$$
\delta \mathcal{L}=\frac{\partial \mathcal{L}}{\partial \varphi_{\rho}} \delta \varphi_{\rho}+\frac{\partial \mathcal{L}}{\partial\left(\partial_{\mu} \varphi_{\rho}\right)} \delta\left(\frac{\partial \varphi_{\rho}}{\partial x^{\mu}}\right)
$$

Deste modo, considerando-se que $\partial_{\mu} \operatorname{comuta} \operatorname{com} \delta$, tem-se que a variação da ação $S$ é dada por:

$$
\delta S=\int\left[\frac{\partial \mathcal{L}}{\partial \varphi_{\rho}} \delta \varphi_{\rho}+\frac{\partial \mathcal{L}}{\partial\left(\partial_{\mu} \varphi_{\rho}\right)}\left(\partial_{\mu} \delta \varphi_{\rho}\right)\right] d^{4} x=0
$$

Utilizando-se na relação acima a igualdade: 


$$
\frac{\partial \mathcal{L}}{\partial\left(\partial_{\mu} \varphi_{\rho}\right)}\left(\partial_{\mu} \delta \varphi_{\rho}\right)=\partial_{\mu}\left[\frac{\partial \mathcal{L}}{\partial\left(\partial_{\mu} \varphi_{\rho}\right)} \delta \varphi_{\rho}\right]-\delta \varphi_{\rho} \partial_{\mu}\left[\frac{\partial \mathcal{L}}{\partial\left(\partial_{\mu} \varphi_{\rho}\right)}\right]
$$

obtém-se a seguinte relação para a variação da ação $S$ :

$$
\delta S=\int\left\{\frac{\partial \mathcal{L}}{\partial \varphi_{\rho}} \delta \varphi_{\rho}+\partial_{\mu}\left[\frac{\partial \mathcal{L}}{\partial\left(\partial_{\mu} \varphi_{\rho}\right)} \delta \varphi_{\rho}\right]-\delta \varphi_{\rho} \partial \mu\left[\frac{\partial \mathcal{L}}{\partial\left(\partial_{\mu} \varphi_{\rho}\right)}\right]\right\} d^{4} x=0
$$

Utilizando-se o teorema de Gauss para o caso quadridimensional, o segundo termo da integral acima se anula, pois $\delta \varphi_{\rho}$ não varia na superfície que delimita o volume quadridimensional. Deste modo, obtém-se:

$$
\left.\delta S=\int\left\{\frac{\partial \mathcal{L}}{\partial \varphi_{\rho}}-\partial \mu \frac{\partial \mathcal{L}}{\partial\left(\partial_{\mu} \varphi_{\rho}\right)}\right]\right\} \delta \varphi_{\rho} d^{4} x=0
$$

Assim, devido à independência de cada variação $\delta \varphi_{\rho}$, chega-se às equações de Euler-Lagrange para os campos $\varphi_{\rho}$ :

$$
\frac{\partial \mathcal{L}}{\partial \varphi_{\rho}}-\partial_{\mu}\left[\frac{\partial \mathcal{L}}{\partial\left(\partial_{\mu} \varphi_{\rho}\right)}\right]=0, \operatorname{com}(\rho=1, \ldots N) \text { e }(\mu=1, \ldots 4)
$$

onde se tem um sistema de $N$ equações diferenciais parciais de segunda ordem que, juntamente com as condições de contorno, permite a obtenção dos $N$ campos $\varphi_{\rho}\left(x^{\mu}\right)$.

A seguir, as equações acima são aplicadas ao campo eletromagnético para se obter a formulação Lagrangiana da eletrodinâmica.

\subsection{A formulação Lagrangiana para o campo eletromagnético.}

$\mathrm{Na}$ formulação Lagrangiana para o campo eletromagnético [Fr-05, Ja-99, Le-07, Go-80], os campos $\varphi_{\rho}\left(x^{\mu}\right)$ são representados pelas quatro componentes do quadrivetor 
potencial, ou seja, $\varphi_{\rho}\left(x^{\mu}\right) \equiv A_{\rho}\left(x^{\mu}\right) \quad(\rho=1, \ldots 4)$. As equações de Euler-Lagrange (3.15) tomam, então, a forma:

$$
\frac{\partial \mathcal{L}}{\partial A_{\nu}}-\partial_{\mu}\left[\frac{\partial \mathcal{L}}{\partial\left(\partial_{\mu} A_{v}\right)}\right]=0(\nu, \mu=1, \ldots 4) .
$$

A densidade da Lagrangiana para o campo eletromagnético é determinada a partir de uma analogia ou comparação com a Lagrangiana de uma partícula carregada num campo eletromagnético [He-95], que é dada por $L=L_{\ell}+L_{I}=-m \sqrt{1-v^{2}}+q \vec{v} \cdot \vec{A}-q \Phi$, onde:

$$
L_{\ell}=-m \sqrt{1-v^{2}}=-m \gamma^{-1}
$$

é a Lagrangiana associada a uma partícula livre de massa de repouso $m$ e velocidade $v$ $(c=1) \mathrm{e}:$

$$
L_{I}=q \vec{v} \cdot \vec{A}-q \Phi
$$

é a Lagrangiana associada à interação da partícula de carga $q$ com o campo dado.

É fácil notar, de (3.17), que $\gamma L_{\ell}$ é um invariante. Por meio da relação acima, tem-se que $\gamma L_{I}=\gamma(q \vec{v} \cdot \vec{A}-q \Phi)=q U^{\alpha} A_{\alpha}$, também é um invariante, onde $U^{\alpha}$ é a quadrivelocidade da partícula.

Passando-se agora para o caso contínuo, como a ação e o elemento de volume quadridimensional são invariantes, segue-se que o mesmo deve ocorrer com a densidade da Lagrangiana $\mathcal{L}$.

Por analogia com o caso da partícula livre, tem-se que $\mathcal{L}$ deve ser quadrática nas velocidades, em $\partial_{\alpha} A_{\beta}$ ou $F_{\alpha \beta}$, e um invariante conhecido [Gr-99], que envolve esses produtos, é $F^{\alpha \beta} F_{\alpha \beta}$.

A partir da Lagrangiana de interação $L_{I}$ e considerando-se que o quadrivetor densidade de corrente $j^{\mu}$ é proporcional ao produto da carga pela quadrivelocidade, ou seja, 
$j^{\alpha} \propto q U^{\alpha}$, tem-se que o termo de interação da densidade da Lagrangiana deve ser proporcional ao escalar $A_{\alpha} j^{\alpha}$. Dessas observações, segue que a densidade da Lagrangiana para o campo eletromagnético é dada por:

$$
\mathcal{L}=-\frac{1}{4} F^{\alpha \beta} F_{\alpha \beta}+A_{\alpha} j^{\alpha}
$$

As constantes em cada um dos termos dependem do sistema de unidades escolhido e da compatibilidade de $\mathcal{L}$ com as equações de Maxwell.

A eletrodinâmica na forma Lagrangiana é dada por $\mathcal{L}$, a densidade da Lagrangiana da eletrodinâmica clássica na forma (3.18), juntamente com o sistema de equações (3.16), ou, de forma equivalente, por (3.18) e pelo princípio de Hamilton. Ou seja, nesse formalismo, toda a eletrodinâmica se resume às equações de Euler-Lagrange e à densidade da Lagrangiana da interação eletromagnética $\mathcal{L}$.

A substituição da densidade da Lagrangiana (3.18) nas equações de Euler-Lagrage (3.16) leva a:

$$
\partial_{\mu}\left(F^{v \mu}\right)=J^{v}
$$

que são as equações não homogêneas de Maxwell (3.9).

As equações homogêneas, dadas por (3.8), são obtidas diretamente das propriedades do tensor eletromagnético, como se verificou anteriormente.

Vê-se, deste modo, que as equações de Maxwell podem ser derivadas de um princípio variacional e uma consequência importante disso é que, se a ação $S$ é invariante sob algum grupo de transformações que atua nas coordenadas e nos campos, então existem quantidades conservadas associadas a estas transformações. Essa relação entre invariância (simetria) e grandezas conservadas é o ponto central do teorema de Noether que tem grande importância em toda a Física e em particular na teoria de campos e partículas. Na próxima seção, aborda-se o teorema de Noether para meios contínuos. 


\subsection{O teorema de Noether para campos.}

Sabe-se que as relações entre as propriedades de simetria ou invariâncias de um sistema físico estão ligadas a leis de conservação e são objeto do teorema de Noether [Go-80, Gr-96, Le-07, No-18, Ta-71]. Examina-se, a seguir, de maneira sucinta, o Teorema de Noether para campos clássicos.

Inicialmente, considera-se uma transformação infinitesimal de coordenadas dada por:

$$
x^{\mu} \rightarrow x^{\prime \mu}=x^{\mu}+\Delta x^{\mu},
$$

onde $\Delta x^{\mu}$ é uma variação infinitesimal que pode ser uma função das coordenadas $x^{v}$.

Essa transformação de coordenadas acarreta uma mudança no campo $\varphi_{\rho}(x)$, expressa por:

$$
\varphi_{\rho}(x) \rightarrow \varphi^{\prime} \rho^{\left(x^{\prime}\right)=\varphi_{\rho}(x)+\Delta \varphi_{\rho}(x), \rho=1, \ldots ., N}
$$

onde $\Delta \varphi_{\rho}(x)$ é a variação total do campo $\varphi_{\rho}(x)$, isto é, uma variação devida tanto a alteração da forma funcional de $\varphi$ como de seu argumento $x$.

As variações local (3.12) e total (3.19) do campo estão relacionadas por:

$$
\delta \varphi_{\rho}(x)=\Delta \varphi_{\rho}(x)-\left[\varphi_{\rho}^{\prime}\left(x^{\prime}\right)-\varphi^{\prime} \rho^{(x)}\right]
$$

Considerando-se que $\varphi_{\rho} \cong \varphi_{\rho}^{\prime}$, pode-se usar a expansão em série de Taylor de $\varphi_{\rho}(x)$ em primeira ordem para o termo entre colchetes da relação acima, obtendo-se:

$$
\delta \varphi_{\rho}(x)=\Delta \varphi_{\rho}(x)-\frac{\partial \varphi_{\rho}(x)}{\partial x^{\mu}} \Delta x^{\mu} .
$$

As transformações infinitesimais de coordenadas e as respectivas mudanças nos campos acarretam uma mudança na densidade da Lagrangiana que é dada por: 


$$
\mathcal{L}^{\prime}\left(x^{\prime}\right)=\mathcal{L}(x)+\Delta \mathcal{L}(x)
$$

onde $\mathcal{L}(x) \equiv \mathcal{L}\left[\varphi_{\rho}(x), \partial_{\mu} \varphi_{\rho}(x)\right]$ e assume-se que $\mathcal{L}(x)$ mantém sua forma funcional na transformação, ou seja, $\mathcal{L}^{\prime}\left[\varphi^{\prime} \rho\left(x^{\prime}\right), \partial_{\mu^{\prime}} \varphi^{\prime} \rho\left(x^{\prime}\right)\right]=\mathcal{L}\left[\varphi^{\prime} \rho\left(x^{\prime}\right), \partial \mu^{\prime} \varphi^{\prime} \rho\left(x^{\prime}\right)\right]$.

Considerando-se que a ação é um invariante sob as transformações de coordenadas e dos campos, tem-se que:

$$
\delta S=S^{\prime}-S=\int_{\Omega^{\prime}} d^{4} x^{\prime} \mathcal{L}^{\prime}\left(x^{\prime}\right)-\int_{\Omega} d^{4} x \mathcal{L}(x)=0
$$

onde $\Omega^{\prime}$ e $\Omega$ denotam os volumes de integração nas coordenadas $x^{\prime}$ e $x$, respectivamente.

A transformação dos volumes de integração é dada utilizando-se o Jacobiano em primeira ordem, de forma que:

$$
d^{4} x^{\prime} \cong\left(1+\frac{\partial \Delta x^{\mu}}{\partial x^{\mu}}\right) d^{4} x
$$

Utilizando-se a variação da densidade da Lagrangiana (3.21) na variação da ação (3.22), tem-se que:

$$
\delta S=\int_{\Omega^{\prime}} d^{4} x^{\prime} \mathcal{L}(x)+\int_{\Omega^{\prime}} d^{4} x^{\prime} \Delta \mathcal{L}(x)-\int_{\Omega} d^{4} x \mathcal{L}(x)=0
$$

Substituindo-se (3.23) nessa relação e desprezando-se o termo de segunda ordem, obtém-se:

$$
\delta S=\int_{\Omega}\left[\mathcal{L}(x) \frac{\partial \Delta x^{\mu}}{\partial x^{\mu}}+\Delta \mathcal{L}(x)\right] d^{4} x=0
$$

As variações total $\Delta \mathcal{L}(x)$ e local $\delta \mathcal{L}(x)$ para a densidade da Lagrangiana são relacionadas pela expressão: 


$$
\Delta \mathcal{L}(x)=\delta \mathcal{L}(x)+\frac{\partial \mathcal{L}(x)}{\partial x^{\mu}} \Delta x^{\mu}
$$

de modo que $\delta S$ fica na forma:

$$
\delta S=\int_{\Omega}\left\{\delta \mathcal{L}(x)+\frac{\partial\left[\mathcal{L}(x) \Delta x^{\mu}\right]}{\partial x^{\mu}}\right\} d^{4} x=0
$$

A variação local da densidade da Lagrangiana $\delta \mathcal{L}(x)$ é dada pela relação (3.14). Subtraindo-se e somando-se, nessa relação, o termo $\frac{\partial}{\partial x^{\mu}}\left(\frac{\partial \mathcal{L}(x)}{\partial\left(\partial_{\mu} \varphi_{\rho}\right)}\right) \delta \varphi_{\rho}$, e aplicando-se a relação de comutação (3.13) entre $\partial_{\mu}$ e $\delta$, obtém-se:

$$
\delta \mathcal{L}(x)=\left[\frac{\partial \mathcal{L}(x)}{\partial \varphi_{\rho}}-\frac{\partial}{\partial x^{\mu}}\left(\frac{\partial \mathcal{L}(x)}{\partial\left(\partial_{\mu} \varphi_{\rho}\right)}\right)\right] \delta \varphi_{\rho}+\frac{\partial}{\partial x^{\mu}}\left(\frac{\partial \mathcal{L}(x)}{\partial\left(\partial_{\mu} \varphi_{\rho}\right)} \delta \varphi_{\rho}\right)
$$

Substituindo-se a relação acima em (3.24), obtém-se:

$$
\delta S=\int_{\Omega}\left\{\left[\frac{\partial \mathcal{L}(x)}{\partial \varphi_{\rho}}-\frac{\partial}{\partial x^{\mu}}\left(\frac{\partial \mathcal{L}(x)}{\partial\left(\partial_{\mu} \varphi_{\rho}\right)}\right)\right] \delta \varphi_{\rho}+\frac{\partial}{\partial x^{\mu}}\left[\frac{\partial \mathcal{L}(x)}{\partial\left(\partial_{\mu} \varphi_{\rho}\right)} \delta \varphi_{\rho}+\mathcal{L}(x) \Delta x^{\mu}\right]\right\} d^{4} x=0
$$

Devido à arbitrariedade da região de integração $\Omega$, o integrando da relação (3.25) é nulo, de forma que:

$$
\left[\frac{\partial \mathcal{L}(x)}{\partial \varphi_{\rho}}-\frac{\partial}{\partial x^{\mu}}\left(\frac{\partial \mathcal{L}(x)}{\partial\left(\partial_{\mu} \varphi_{\rho}\right)}\right)\right] \delta \varphi_{\rho}+\frac{\partial}{\partial x^{\mu}}\left[\frac{\partial \mathcal{L}(x)}{\partial\left(\partial_{\mu} \varphi_{\rho}\right)} \delta \varphi_{\rho}+\mathcal{L}(x) \Delta x^{\mu}\right]=0 .
$$

O primeiro termo da expressão acima é a equação de Euler-Lagrange (3.15) para campos, que é identicamente nulo. Portanto tem-se que:

$$
\frac{\partial}{\partial x^{\mu}}\left[\frac{\partial \mathcal{L}(x)}{\partial\left(\partial \mu \varphi_{\rho}\right)} \delta \varphi_{\rho}+\mathcal{L}(x) \Delta x \mu\right]=0
$$


Utilizando-se (3.20) para as variações $\delta \varphi_{\rho}(x)$ e $\Delta \varphi_{\rho}(x)$ na relação acima, obtémse:

$$
\frac{\partial}{\partial x^{\mu}}\left\{\frac{\partial \mathcal{L}(x)}{\partial\left(\partial_{\mu} \varphi_{\rho}\right)} \Delta \varphi_{\rho}(x)-\left[\frac{\partial \mathcal{L}(x)}{\partial\left(\partial_{\mu} \varphi_{\rho}\right)} \frac{\partial \varphi_{\rho}(x)}{\partial x^{\nu}}-\mathcal{L}(x) \delta_{v}^{\mu}\right] \Delta x^{v}\right\}=0
$$

Esta expressão é uma equação de continuidade na forma:

$$
\frac{\partial f^{\mu}(x)}{\partial x^{\mu}}=0
$$

que define a seguinte densidade de corrente:

$$
f^{\mu}(x)=\frac{\partial \mathcal{L}(x)}{\partial\left(\partial_{\mu} \varphi_{\rho}\right)} \Delta \varphi_{\rho}(x)-\left[\frac{\partial \mathcal{L}(x)}{\partial\left(\partial_{\mu} \varphi_{\rho}\right)} \frac{\partial \varphi_{\rho}(x)}{\partial x^{v}}-\mathcal{L}(x) \delta_{v}^{\mu}\right] \Delta x^{v}
$$

Contudo, é mais conveniente expressar-se a equação de continuidade e a densidade de corrente $f^{\mu}$ em termos de $R$ parâmetros infinitesimais, independentes e constantes $\varepsilon^{r}$, com $(r=1,2, \ldots, R)$, onde as variações nas coordenadas e nos campos são lineares em $\varepsilon^{r}$, nas formas:

$$
\Delta x^{\mu}=\xi_{r}^{\mu}(x) \varepsilon^{r}
$$

e

$$
\Delta \varphi_{\rho}(x)=\Psi_{\rho r}(x) \varepsilon^{r}
$$

com $\xi_{r}^{\mu}(x), \Psi_{\rho r}(x)$ e $R$ escolhidos de acordo com a grandeza conservada. Por exemplo, para conservação do momento e energia, tem-se que $R=4$.

Deste modo, a condição de invariância da ação (3.25) fica na forma:

$$
\delta S=\int_{\Omega} \varepsilon^{r} \frac{\partial}{\partial x^{\mu}}\left\{\frac{\partial \mathcal{L}(x)}{\partial\left(\partial_{\mu} \varphi_{\rho}\right)} \Psi_{\rho r}(x)-\left[\frac{\partial \mathcal{L}(x)}{\partial\left(\partial_{\mu} \varphi_{\rho}\right)} \frac{\partial \varphi_{\rho}(x)}{\partial x^{\nu}}-\mathcal{L}(x) \delta_{V}^{\mu}\right] \xi_{r}(x)\right\} d^{4} x=0
$$


O que implica na equação de continuidade:

$$
\frac{\partial}{\partial x^{\mu}}\left\{\frac{\partial \mathcal{L}(x)}{\partial\left(\partial_{\mu} \varphi_{\rho}\right)} \Psi_{\rho r}(x)-\left[\frac{\partial \mathcal{L}(x)}{\partial\left(\partial_{\mu} \varphi_{\rho}\right)} \frac{\partial \varphi_{\rho}(x)}{\partial x^{v}}-\mathcal{L}(x) \delta_{v}^{\mu}\right] \xi_{r}^{V}(x)\right\}=0
$$

Assim, tem-se a densidade de corrente redefinida em termos das funções $\Psi$ e $\xi$ :

$$
f_{r}^{\mu}(x)=\frac{\partial \mathcal{L}(x)}{\partial\left(\partial_{\mu} \varphi_{\rho}\right)} \Psi_{\rho r}(x)-\left[\frac{\partial \mathcal{L}(x)}{\partial\left(\partial_{\mu} \varphi_{\rho}\right)} \frac{\partial \varphi_{\rho}(x)}{\partial x^{V}}-\mathcal{L}(x) \delta_{V}^{\mu}\right] \xi_{r}^{V}(x)
$$

Considerando-se a relação acima, a integração da equação diferencial (3.28) no espaço implica que:

$$
\int_{V} d^{3} x \frac{\partial f_{r}^{\mu}(x)}{\partial x^{\mu}}=\int_{V} d^{3} x \vec{\nabla} \cdot \vec{f}_{r}(x)+\int_{V} d^{3} x \frac{\partial f_{r}^{4}(x)}{\partial x^{4}}=0
$$

Utilizando-se o teorema de Gauss no primeiro termo desta soma, tem-se que:

$$
\int_{V} d^{3} x \frac{\partial f_{r}^{\mu}(x)}{\partial x^{\mu}}=\oint_{S} \vec{f}_{r}(x) \cdot d \vec{S}+\frac{d}{d x^{4}} \int_{V} d^{3} x f_{r}^{4}(x)=0
$$

A integral de superfície da relação acima se anula, pois os campos e suas derivadas tendem a zero quando o volume considerado é todo o espaço tridimensional. Assim, tem-se que:

$$
\int_{V} d^{3} x \frac{\partial f_{r}^{\mu}(x)}{\partial x^{\mu}}=\frac{d}{d x^{4}} \int_{V} d^{3} x f_{r}^{4}(x)=0
$$

As grandezas conservadas, isto é, que independem do tempo, são dadas, então, por:

$$
Q_{r}=\int_{V} d^{3} x f_{r}^{4}(x),
$$


e são denominadas cargas de Noether.

Este é o principal resultado do teorema de Noether, que associa a uma simetria contínua uma lei de conservação.

Esse teorema também é aplicável a sistemas mecânicos discretos, onde se tem a troca da integral quadridimensional da ação pela integral unidimensional em $t, \delta S=\delta \int L d t$. As variáveis contínuas (campos), $\varphi_{\rho}\left(x^{\mu}\right)$, são substituídas pelas coordenadas generalizadas $q_{k}(t)$ e as derivadas $\partial_{\mu} \varphi_{\rho}$ por $\dot{q}_{k}(t)$. Nessas condições, tem-se que [Go-80, Le-07]:

$$
\frac{d}{d t}\left\{\left(\frac{\partial L}{\partial \dot{q}_{k}} \dot{q}_{k}-L\right) \delta t-\frac{\partial L}{\partial \dot{q}_{k}} \delta q_{k}\right\}=0
$$

\subsection{Aplicação do teorema de Noether para o campo eletromagnético.}

Nesta seção, aplicam-se os resultados do teorema de Noether para o campo eletromagnético [Al-06, Be-21, Pl-74]. Neste caso, considera-se a densidade da Lagrangiana para o campo eletromagnético livre, dada por:

$$
\mathcal{L}=-\frac{1}{4} F^{\alpha \beta} F_{\alpha \beta}
$$

Substituindo-se $\varphi_{\rho}(x)$ por $A_{v}(x)$ na relação (3.28), obtém-se:

$$
\frac{\partial}{\partial x^{\mu}}\left\{\frac{\partial \mathcal{L}(x)}{\partial\left(\partial_{\mu} A_{V}(x)\right)} \Psi_{\nu r}(x) \mathcal{E}^{r}-\left[\frac{\partial \mathcal{L}(x)}{\partial\left(\partial_{\mu} A_{V}(x)\right)} \frac{\partial A_{\nu}(x)}{\partial x^{\lambda}}-\mathcal{L}(x) \delta_{\lambda}^{\mu}\right] \xi_{r}^{\lambda}(x) \mathcal{E}^{r}\right\}=0
$$

onde a densidade da Lagrangiana é dada pela relação (3.31) e as variações totais das coordenadas e dos campos são dadas, respectivamente, por (3.26) e (3.27).

Em relação às coordenadas $x$ e $x^{\prime}$, tem-se que o quadrivetor potencial $A_{v}$ obedece à seguinte lei de transformação:

$$
A_{v}(x)=\frac{\partial x^{\prime} \mu}{\partial x^{v}} A^{\prime} \mu^{\left(x^{\prime}\right)}
$$


Substituindo-se as transformações de coordenadas infinitesimais $x^{\prime \mu}=x^{\mu}+\xi_{r}^{\mu}(x) \varepsilon^{r}$ na expressão acima, tem-se que:

$$
A_{v}^{\prime}\left(x^{\prime}\right)-A_{v}(x)=-A^{\prime} \mu^{\left(x^{\prime}\right)} \frac{\partial\left[\xi_{r}^{\mu}(x) \varepsilon^{r}\right]}{\partial x^{V}}
$$

Lembrando-se que a variação total $\Delta A_{v}$ é dada por $\Delta A_{v}=\Psi_{v r}(x) \varepsilon^{r}=A_{v}^{\prime}\left(x^{\prime}\right)-A_{v}(x)$ e considerando-se que em primeira ordem $A_{v}^{\prime}\left(x^{\prime}\right) \cong A_{v}(x)$, a relação acima implica que:

$$
\Delta A_{v}=\Psi_{v r}(x) \varepsilon^{r}=-A_{\sigma}(x) \frac{\partial\left[\xi_{r}^{\sigma}(x) \varepsilon^{r}\right]}{\partial x^{V}}
$$

Substituindo-se a relação acima em (3.32) e rearranjando-se os termos, tem-se que:

$$
\frac{\partial}{\partial x^{\mu}}\left\{\mathcal{L}(x) \xi_{r}^{\mu}(x) \mathcal{E}^{r}-\frac{\partial \mathcal{L}(x)}{\partial\left(\partial_{\mu} A_{\nu}(x)\right)}\left[A_{\sigma}(x) \frac{\partial\left[\xi_{r}^{\sigma}(x) \mathcal{E}^{r}\right]}{\partial x^{\nu}}+\frac{\partial A_{\nu}(x)}{\partial x^{\lambda}} \xi_{r}^{\lambda}(x) \varepsilon^{r}\right]\right\}=0
$$

Para o campo eletromagnético, obtém-se:

$$
\frac{\partial \mathcal{L}(x)}{\partial\left(\partial_{\mu} A_{v}\right)}=-F^{\mu v}
$$

Substituindo-se a relação acima em (3.33) e trocando-se o índice mudo $\lambda$ por $\sigma$, tem-se que:

$$
\frac{\partial}{\partial x^{\mu}}\left\{\mathcal{L}(x) \xi_{r}^{\mu}(x) \mathcal{E}^{r}+F^{\mu \nu} A_{\sigma}(x) \frac{\partial\left[\xi_{r}^{\sigma}(x) \varepsilon^{r}\right]}{\partial x^{\nu}}+F^{\mu \nu} \frac{\partial A_{\nu}(x)}{\partial x^{\sigma}} \xi_{r}^{\sigma}(x) \mathcal{E}^{r}\right\}=0 .
$$

O segundo termo da soma entre chaves pode ser expresso do seguinte modo:

$$
F^{\mu v} A_{\sigma}(x) \frac{\partial\left[\xi_{r}^{\sigma}(x) \varepsilon^{r}\right]}{\partial x^{V}}=F^{\mu v} \frac{\partial\left[A_{\sigma}(x) \xi_{r}^{\sigma}(x) \varepsilon^{r}\right]}{\partial x^{V}}-\frac{\partial\left[A_{\sigma}(x)\right]}{\partial x^{V}} \xi_{r}^{\sigma}(x) \varepsilon^{r}
$$


Assim, substituindo-se esta expressão em (3.34) e considerando-se que o tensor eletromagnético em sua forma covariante é dado por $F_{v \sigma}=\partial_{v} A_{\sigma}-\partial_{\sigma} A_{v}$, obtém-se:

$$
\frac{\partial}{\partial x^{\mu}}\left\{\xi_{r}^{\sigma}(x) \mathcal{E}^{r}\left[\mathcal{L}(x) \delta_{\sigma}^{\mu}-F^{\mu \nu} F_{\nu \sigma}\right]+F^{\mu \nu} \frac{\partial\left[A_{\sigma}(x) \xi_{r}^{\sigma}(x) \mathcal{E}^{r}\right]}{\partial x^{v}}\right\}=0
$$

Sabe-se que o tensor de energia-momento simétrico [Fr-05, Ja-99, Le-07] do campo eletromagnético livre é dado por:

$$
\Theta^{\mu \lambda}=\mathcal{L}(x) \eta^{\mu \lambda}-F^{\mu v} F_{v}^{\lambda}=-\frac{1}{4} \eta^{\mu \lambda} F^{\alpha \beta} F_{\alpha \beta}-F^{\mu v} F_{v}^{\lambda}
$$

onde: $F_{v}^{\lambda}=F_{v \sigma} \eta^{\sigma \lambda}$

Deste modo, o primeiro termo da expressão (3.35) relaciona-se com o tensor de energia momento simétrico $\Theta^{\mu \lambda}$ da seguinte forma:

$$
\Theta_{\sigma}^{\mu}=\Theta^{\mu \lambda} \eta_{\lambda \sigma}=\mathcal{L}(x) \delta_{\sigma}^{\mu}-F^{\mu v} F_{v \sigma}
$$

onde as componentes destes tensores satisfazem:

$$
\Theta_{\sigma}^{\mu}=\Theta^{\mu \sigma} \text {, para } \sigma=1,2,3 \quad \text { e } \quad \Theta^{\mu}{ }_{\sigma}=-\Theta^{\mu \sigma} \text {, para } \sigma=4 \text {. }
$$

Assim, tem-se que (3.35) fica na forma:

$$
\frac{\partial}{\partial x^{\mu}}\left\{\Theta^{\mu} \xi_{r}(x) \varepsilon^{r}+F^{\mu \nu} \frac{\partial\left[A_{\sigma}(x) \xi_{r}(x) \varepsilon^{r}\right]}{\partial x^{v}}\right\}=0
$$

Desenvolvendo-se a expressão acima, obtém-se:

$$
\frac{\partial\left[\Theta^{\mu}{ }_{\sigma} \xi_{r}^{\sigma}(x) \mathcal{E}^{r}\right]}{\partial x^{\mu}}+\frac{\partial F^{\mu \nu}}{\partial x^{\mu}} \frac{\partial\left[A_{\sigma}(x) \xi_{r}^{\sigma}(x) \varepsilon^{r}\right]}{\partial x^{V}}+F^{\mu \nu} \frac{\partial}{\partial x^{\mu}} \frac{\partial\left[A_{\sigma}(x) \xi_{r}^{\sigma}(x) \mathcal{E}^{r}\right]}{\partial x^{V}}=0
$$


O segundo termo desta soma anula-se, pois, para o campo eletromagnético livre, tem-se que: $\frac{\partial F^{\mu v}}{\partial x^{\mu}}=0$.

Deste modo, a relação acima fica na forma:

$$
\frac{\partial\left[\Theta^{\mu}{ }_{\sigma} \xi_{r}^{\sigma}(x) \varepsilon^{r}\right]}{\partial x^{\mu}}+F^{\mu \nu} \frac{\partial}{\partial x^{\mu}} \frac{\partial\left[A_{\sigma}(x) \xi_{r}^{\sigma}(x) \varepsilon^{r}\right]}{\partial x^{V}}=0 .
$$

Devido à anti-simetria de $F^{\mu \nu}$, o segundo termo desta soma anula-se e, considerando-se a independência dos $R$ parâmetros $\mathcal{E}^{r}$, tem-se que as $R$ correntes conservadas são dadas pela equação:

$$
\frac{\partial\left[\Theta^{\mu} \xi_{r}^{\sigma}(x)\right]}{\partial x^{\mu}}=0
$$

$\operatorname{com} \Theta_{\sigma}^{\mu}$ dado por (3.36).

A determinação das leis de conservação associadas à invariância conforme do campo eletromagnético é feita a partir da substituição das funções $\xi_{r}^{\mu}(x)$, relativas às transformações conformes no espaço-tempo, na quadridivergência acima.

Utilizando-se (3.29) para o caso do campo eletromagnético, tem-se que as cargas de Noether conservadas são dadas por:

$$
Q_{r}=\int_{V} d^{3} x \Theta^{4} \sigma \xi_{r}^{\sigma}(x)
$$

No próximo capítulo, serão abordadas as transformações conformes em espaços planos de $n$ dimensões e determinadas as respectivas funções $\xi_{r}^{\mu}(x)$ associadas às transformações conformes no espaço-tempo. 


\section{CAPÍTULO IV}

\section{TRANSFORMAÇÕES CONFORMES EM ESPAÇOS PLANOS DE N DIMENSÕES.}

Neste capítulo, determinam-se e discutem-se as transformações conformes de coordenadas em espaços planos de $n$ dimensões. Como foi exposto na parte histórica do Capítulo II, os aspectos aqui abordados foram tratados originalmente por Liouville para espaços euclidianos tridimensionais e generalizados por Lie para espaços euclidianos e não euclidianos $n$-dimensionais. Em essência, o desenvolvimento abaixo segue as idéias desses trabalhos, que mostram que há restrições para as transformações conformes para espaços de dimensão maior ou igual a três.

O capítulo inicia-se com a determinação das condições matemáticas para que uma transformação infinitesimal de coordenadas seja conforme em espaços planos $n$-dimensionais. Aplicam-se, logo após, as condições obtidas para a determinação das transformações conformes no plano complexo $(n=2)$ e em espaços planos com dimensão $n \geq 3$. Finalmente, discutem-se as transformações conformes no espaço-tempo de Minkowski $(n=4)$ e, em seguida, as transformações de Lorentz e de Poincaré como são expostas em textos sobre a teoria da relatividade.

\subsection{Condições para as transformações conformes em espaços planos de $\boldsymbol{n}$ dimensões.}

Sejam as transformações infinitesimais de coordenadas na forma:

$$
x^{\prime \mu}=x^{\mu}+\varepsilon^{\mu}(x)+O\left(\varepsilon^{2}\right), \mu=1, \ldots n
$$

onde $n$ é a dimensão do espaço e se desprezam os termos de segunda ordem.

Determinam-se, a seguir, as condições para que as transformações infinitesimais (4.1) sejam conformes [B1-09, Fr-97], ou seja, quais as restrições que devem ser impostas à forma das funções $\varepsilon^{\mu}(x)$ para que a relação (2.17) seja satisfeita.

Como se verificou anteriormente, se o espaço é plano, tem-se que $\eta_{\mu v}= \pm l(\mu=v)$ e $\eta_{\mu v}=O(\mu \neq v)$, e a substituição de (4.1) em (2.17) leva à relação: 


$$
\partial_{\alpha} \varepsilon_{\beta}+\partial_{\beta} \varepsilon_{\alpha}=k(x) \eta_{\alpha \beta}
$$

onde a função $k(x)$ está ligada ao fator de escala $\sigma(x)$ pela relação:

$$
\sigma(x)=1+k(x)+O\left(\varepsilon^{2}\right) .
$$

A função $k(x)$ pode ser determinada multiplicando-se ambos os lados de (4.2) por $\eta^{\alpha \beta}$. Igualando-se os traços das matrizes resultantes, obtém-se:

$$
k(x)=\frac{2 \partial^{\alpha} \varepsilon_{\alpha}}{n}
$$

Utilizando-se a expressão acima na relação (4.2), tem-se a seguinte restrição para que a transformação (4.1) seja conforme num espaço plano:

$$
\partial_{\alpha} \varepsilon_{\beta}+\partial_{\beta} \varepsilon_{\alpha}=\frac{2}{n}(\partial . \varepsilon) \eta_{\alpha \beta}
$$

onde se utilizou a notação $\partial . \varepsilon=\partial^{\alpha} \varepsilon_{\alpha}$.

Tomando-se a derivada $\partial^{\beta}=\frac{\partial}{\partial x_{\beta}}$ da equação (4.4), tem-se que:

$$
\partial_{\alpha}(\partial . \varepsilon)+\square \varepsilon_{\alpha}=\frac{2}{n} \partial_{\alpha}(\partial . \varepsilon)
$$

onde $\square=\partial^{\alpha} \partial_{\alpha}$.

Tomando-se agora a derivada $\partial_{\beta}$ de ambos os lados da igualdade acima, tem-se que:

$$
\partial_{\alpha} \partial_{\beta}(\partial . \varepsilon)+\square \partial{ }_{\beta} \varepsilon_{\alpha}=\frac{2}{n} \partial_{\alpha} \partial_{\beta}(\partial . \varepsilon) .
$$


Comutando-se os índices $\alpha$ e $\beta$ na relação acima e somando-se o resultado a ela mesma, obtém-se:

$$
\left(\partial_{\alpha} \partial_{\beta}+\partial_{\beta} \partial_{\alpha}\right)(\partial . \varepsilon)+\square\left(\partial_{\beta} \varepsilon_{\alpha}+\partial_{\alpha} \varepsilon_{\beta}\right)=\frac{2}{n}\left(\partial_{\alpha} \partial_{\beta}+\partial_{\beta} \partial_{\alpha}\right)\left(\partial_{. \varepsilon}\right) .
$$

Substituindo-se o segundo termo da soma à esquerda dessa relação pela expressão (4.4) e considerando-se que $\partial_{\alpha} \partial_{\beta}=\partial_{\beta} \partial_{\alpha}$, tem-se:

$$
\left[\eta_{\alpha \beta} \square+(n-2) \partial_{\alpha} \partial_{\beta}\right](\partial . \varepsilon)=0
$$

Contraindo-se a relação acima com $\eta^{\alpha \beta}$ obtém-se a restrição na forma:

$$
(n-1) \square(\partial . \varepsilon)=0
$$

A seguir, toma-se a derivada $\partial_{\mu}$ da equação (4.4) e permutam-se os índices $\mu, \alpha$ e $\beta$, obtendo-se as três relações seguintes:

$$
\begin{aligned}
& \partial_{\mu} \partial_{\alpha} \varepsilon_{\beta}+\partial_{\mu} \partial_{\beta} \varepsilon_{\alpha}=\frac{2}{n} \eta_{\alpha \beta} \partial_{\mu}(\partial . \varepsilon), \\
& \partial_{\beta} \partial_{\mu} \varepsilon_{\alpha}+\partial_{\alpha} \partial_{\mu} \varepsilon_{\beta}=\frac{2}{n} \eta_{\mu \alpha} \partial_{\beta}(\partial . \varepsilon)
\end{aligned}
$$

$\mathrm{e}$

$$
\partial_{\alpha} \partial_{\beta} \varepsilon_{\mu}+\partial_{\beta} \partial_{\alpha} \varepsilon_{\mu}=\frac{2}{n} \eta_{\beta \mu} \partial_{\alpha}(\partial . \varepsilon) .
$$

Uma nova forma da restrição é obtida subtraindo-se (4.6) da soma de (4.7) com (4.8):

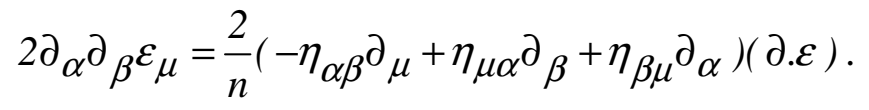


Nos itens que se seguem, serão utilizadas as relações (4.4), (4.5) e (4.9) como condições que devem ser impostas para que a transformação (4.1) seja conforme, ou seja, relações que permitem determinar as funções $\varepsilon^{\mu}(x)$.

\subsection{As transformações conformes para $n=2$.}

Nesta seção, como exemplo e aplicação dos resultados obtidos na seção anterior, apresentam-se as condições para transformações conformes no plano complexo $(n=2)$.

As transformações conformes em duas dimensões são obtidas diretamente da relação (4.4) para $n=2$, ou seja:

$$
\partial_{\alpha} \varepsilon_{\beta}+\partial_{\beta} \varepsilon_{\alpha}=(\partial . \varepsilon) \eta_{\alpha \beta}
$$

O plano complexo é um plano euclidiano e, neste caso, o tensor métrico $\eta_{\alpha \beta}$ é dado por: $\eta_{\alpha \beta}=0$, para $\alpha \neq \beta$, e $\eta_{\alpha \beta}=1$, para $\alpha=\beta=1,2$.

A condição aplicada para $\alpha=\beta=1,2$ implica na igualdade $\partial_{1} \varepsilon_{1}+\partial_{1} \varepsilon_{1}=\partial_{2} \varepsilon_{2}+\partial_{2} \varepsilon_{2}$, ou seja:

$$
\partial_{1} \varepsilon_{1}=\partial_{2} \varepsilon_{2}
$$

A condição para $\alpha \neq \beta$ implica na relação $\partial_{\alpha} \varepsilon_{\beta}+\partial_{\beta} \varepsilon_{\alpha}=0$, isto é:

$$
\partial_{1} \varepsilon_{2}=-\partial_{2} \varepsilon_{1}
$$

As relações (4.10) e (4.11) são as equações de Cauchy-Riemann, ou seja, são as expressões (2.2) apresentadas no Capítulo II, onde se consideram números $\zeta\left(x^{1}, x^{2}\right)=x^{1}+i x^{2}$ no plano $\zeta$. Neste caso, uma transformação conforme infinitesimal de $\zeta$ para $\zeta+\varepsilon(\zeta)$ no plano complexo está associada à função:

$$
f(\zeta)=\zeta+\varepsilon(\zeta)=\left(x^{1}+\varepsilon^{1}\right)+i\left(x^{2}+\varepsilon^{2}\right), \text { onde } \varepsilon=\varepsilon^{1}+i \varepsilon^{2}
$$


De acordo com as relações (4.10) e (4.11), a função $\mathcal{E}(\zeta)$ é analítica e sua expansão em série de Laurent (infinitos coeficientes), indica que o número de transformações conformes no plano $(n=2)$ é infinito.

\subsection{As transformações conformes para $n \geq 3$.}

A seguir, aplicam-se as condições obtidas no item 4.1 para se obter as transformações conformes em espaços planos com dimensão $n \geq 3$. Para isso, parte-se da análise da relação (4.5). Nessa situação, tem-se que:

$$
\square(\partial . \varepsilon)=\partial^{\alpha} \partial_{\alpha} \partial . \varepsilon=0
$$

A relação acima implica que $(\partial . \varepsilon)=A+B_{\mu} x^{\mu}$, com $A$ e $B_{\mu}$ constantes. Assim, temse que $\varepsilon_{\mu}$ é quadrático em $x^{\beta}$, ou seja, tem a forma:

$$
\varepsilon_{\mu}(x)=a_{\mu}+b_{\mu v} x^{v}+c_{\mu v \alpha} x^{v} x^{\alpha}
$$

onde $\left|a_{\mu}\right|,\left|b_{\mu v}\right|,\left|c_{\mu v \alpha}\right| \ll<1$ são constantes e $c_{\mu v \alpha}=c_{\mu \alpha \nu}$.

A expressão acima será utilizada na determinação das transformações conformes infinitesimais de coordenadas em espaços planos de dimensão $n \geq 3$. Para tanto, faz-se necessário a determinação e a interpretação das constantes $a_{\mu}, b_{\mu \nu}$ e $c_{\mu v \alpha}$.

A constante $a_{\mu}$ não está restrita à condição (4.4), pois a sua substituição nessa relação implica que todas as derivadas parciais se anulam. Assim, $a_{\mu}$ descreve as translações infinitesimais:

$$
x^{\prime \mu}=x^{\mu}+a^{\mu} .
$$

A constante $b_{\mu v}$ é determinada quando se insere o termo linear em $x^{\mu}$ da expressão (4.12) na relação (4.4). Neste caso, obtém-se a relação: 


$$
b_{v \mu}+b_{\mu v}=\frac{2}{n}\left(\eta^{\mu v} b_{v \mu}\right) \eta_{\mu v}
$$

Como, neste caso, $\eta_{\mu v}=0$ (para $\left.\mu \neq v\right)$ e $\eta_{\mu v}= \pm l$ (para $\left.\mu=v\right)$, tem-se que a relação (4.14) implica que:

$$
b_{\mu v}=-b_{\nu \mu}, \text { para } \mu \neq v \quad \text { e } \quad b_{\mu v}=\lambda \eta_{\mu v}, \text { para } \mu=v,
$$

onde $\lambda$ é uma constante.

Assim, a matriz constante $b_{\mu \nu}$ é dada por uma parte simétrica e outra anti-simétrica:

$$
b_{\mu v}=\lambda \eta_{\mu v}+m_{\mu v}, \text { onde: } m_{\mu v}=-m_{v \mu} .
$$

A parte anti-simétrica $\left(m_{\mu v}\right)$ da relação acima corresponde a rotações infinitesimais:

$$
x^{\prime \mu}=\left(\delta_{v}^{\mu}+\eta^{\mu \lambda} m_{\lambda v}\right) x^{v},
$$

enquanto a parte simétrica de (4.15), ou seja, o termo $\lambda \eta_{\mu v}$, descreve transformações infinitesimais de escala:

$$
x^{\prime \mu}=(1+\lambda) x^{\mu}
$$

Assim, verifica-se que o termo $b_{\mu \nu}$ define as transformações infinitesimais de rotação e de escala num espaço plano de $n$ dimensões.

Para a constante $c_{\mu v \alpha}$, considera-se, primeiramente, a derivada $\partial . \varepsilon$ da equação (4.12), obtendo-se:

$$
\partial . \varepsilon=b_{\mu}^{\mu}+2 c_{\mu \alpha}^{\mu} x^{\alpha}
$$

Dessa relação, tem-se que: 


$$
\partial_{v}(\partial . \varepsilon)=2 c_{\mu \nu}^{\mu}
$$

Substituindo-se (4.12) em (4.9) e considerando-se a relação acima, obtém-se a expressão:

$$
4 c_{\mu v \alpha}=\frac{4}{n}\left(-\eta_{v \alpha} c_{v \mu}^{v}+\eta_{\mu \nu} c_{v \alpha}^{v}+\eta_{\mu \alpha} c_{v \nu}^{v}\right)
$$

Assim, tem-se que a constante $c_{\mu \nu \alpha}$ é dada por:

$$
c_{\mu v \alpha}=\eta_{\mu \alpha} s_{v}+\eta_{\mu v} s_{\alpha}-\eta_{v \alpha} s_{\mu}
$$

onde: $s_{\mu}=\frac{1}{n} c^{\alpha} \alpha \mu$.

A transformação resultante tem a forma:

$$
x^{\prime \mu}=x^{\mu}+\left(2 x^{\beta} x^{\mu}-x_{v} x^{v} \eta^{\mu \beta}\right) s_{\beta},
$$

e é chamada transformação conforme especial.

Em síntese, as transformações conformes infinitesimais de coordenadas num espaço plano de $n$ dimensões ( $n \geq 3$ ) são dadas por:
a) Translações (4.13):
$x^{\prime \mu}=x^{\mu}+a^{\mu}$,
b) Rotações (4.16):
$x^{\prime \mu}=\left(\delta_{v}^{\mu}+\eta^{\mu \lambda} m_{\lambda v}\right) x^{v}$,
c) Dilatações (4.17):
$x^{\prime \mu}=(1+\lambda) x^{\mu} \quad \mathrm{e}$
d) Transformações conformes especiais (4.18): $x^{\prime}{ }^{\mu}=x^{\mu}+\left(2 x^{\beta} x^{\mu}-x_{v} x^{\nu} \eta^{\mu \beta}\right) s_{\beta}$,

com um total de $\frac{(n+1)(n+2)}{2}$ constantes.

A partir das transformações conformes infinitesimais, chega-se ao grupo de transformações conformes finitas para $n \geq 3$ [Ro-72], que têm a forma: 
a) Translações:

$$
x^{\prime \mu}=x^{\mu}+a^{\mu},
$$

b) Rotações:

$$
x^{\prime \mu}=M_{v}^{\mu} x^{v}
$$

c) Dilatações:

$$
x^{\prime \mu}=\lambda x^{\mu} \quad \mathrm{e}
$$

d) Transformações conformes especiais: $\quad x^{\prime} \mu=\frac{x^{\mu}-(x . x) s^{\mu}}{1-2(\text { s.x })+(\text { s.s })(x . x)}$.

Verifica-se que (4.18) pode facilmente ser obtida da relação acima para as transformações conformes especiais, a partir de uma expansão em série de Taylor para $s^{\mu}<<1$.

É interessante notar que as dilatações e as transformações conformes especiais podem ser entendidas em termos de inversões.

Conforme mencionado no capítulo II (Fig. 2.9), a inversão de dois pontos $P$ e $P^{\prime}$ em relação a uma circunferência de raio $a$ e centro $O$ é dada pela relação $\overline{O P} \cdot \overline{O P}{ }^{\prime}=a^{2}$. Considerando-se o centro na origem $O$ do plano cartesiano, tem-se que a transformação de $\left(x^{1}, x^{2}\right)$ para $\left(x^{\prime}, x^{\prime 2}\right)$ é dada por [B1-00]:

$$
x^{\prime \mu}=\frac{a^{2} x^{\mu}}{\left(x^{1}\right)^{2}+\left(x^{2}\right)^{2}}, \mu=1,2 .
$$

A relação acima pode ser generalizada para espaços planos de $n$ dimensões, de forma que a inversão de $x^{\mu}$ para $x^{\prime \mu}$ é dada por:

$$
x^{\prime \mu}=\frac{a^{2} x^{\mu}}{x . x}
$$

onde: $x \cdot x=\eta_{\mu v} x^{\mu} x^{v}$

As dilatações podem ser entendidas como uma sequência de duas inversões em relação a raios diferentes $a$ e $b$, ou seja:

$$
x \rightarrow x^{\prime \mu}=\frac{a^{2} x^{\mu}}{x \cdot x} \rightarrow x^{\prime \prime} \mu=\frac{b^{2} x^{\prime \mu}}{x^{\prime} \cdot x^{\prime}}=\frac{b^{2} x^{\mu}}{a^{2}}=\lambda x^{\mu}, \operatorname{com} \lambda=\frac{b^{2}}{a^{2}}
$$


Para se chegar às transformações conformes especiais, parte-se da inversão de $x^{\mu}$, com raio unitário, seguida de uma translação de $s^{\mu}$ :

$$
\frac{x^{\mu}}{x \cdot x}-s^{\mu}
$$

e, por fim, faz-se uma nova inversão também com $a=1$, ou seja:

$$
\frac{\frac{x^{\mu}}{x . x}-s^{\mu}}{\left(\frac{x^{\mu}}{x . x}-s^{\mu}\right) \cdot\left(\frac{x^{\mu}}{x . x}-s^{\mu}\right)}=\frac{x^{\mu}-(x . x) s^{\mu}}{1-2(s . x)+(\text { s.s })(x . x)} .
$$

Esse processo é ilustrado na Fig. 4.1.

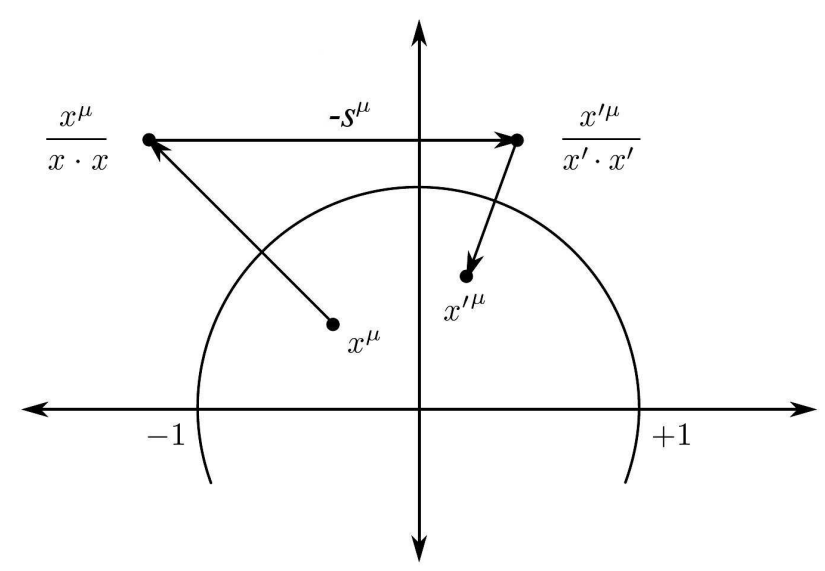

Fig. 4.1 - A transformação conforme especial é composta por uma inversão, seguida de uma translação e de outra inversão [B1-09].

\subsection{As transformações conformes no espaço-tempo.}

Examinam-se agora as transformações conformes no espaço-tempo [Pl-74], bem como os parâmetros que as compõem. Parte-se das transformações (4.13), (4.16), (4.17) e (4.18) para se chegar ao grupo de transformações conformes no espaço-tempo, formado por translações, rotações, dilatações e transformações conformes especiais, onde se utiliza o 
tensor métrico de Minkowski, dado por: $\eta_{\mu v}=1(\mu=v=1,2,3), \eta_{\mu v}=-1(\mu=v=4)$ e $\eta_{\mu v}=0(\mu \neq v)$

Considerando-se as transformações infinitesimais de coordenadas (4.1), tem-se que as funções $\varepsilon^{\mu}(x)$ podem ser dadas em termos de $R$ funções $\xi_{r}^{\mu}(x)(r=1, \ldots, R)$ e dos parâmetros $\varepsilon^{r}<<1$, como usado em (3.26), de modo que (4.1) fique na forma:

$$
x^{\prime \mu}=x^{\mu}+\xi_{r}^{\mu}(x) \varepsilon^{r}+O\left(\varepsilon^{2}\right) .
$$

Os parâmetros $\varepsilon^{r}$ e as funções $\xi_{r}^{\mu}(x)$ relativos a cada transformação conforme infinitesimal no espaço-tempo são obtidos comparando-se a expressão acima com as relações (4.13), (4.16), (4.17) e (4.18).

a) Para as translações, comparando-se (4.13) com (4.23), tem-se que $r=1,2,3,4$. Os parâmetros $\varepsilon^{r}$ são dados por, $\varepsilon^{l}=a^{l}, \varepsilon^{2}=a^{2}, \varepsilon^{3}=a^{3}$ e $\varepsilon^{4}=a^{4}$, enquanto as funções $\xi_{r}^{\mu}(x)$ são dadas por:

$$
\xi_{r}^{\mu}(x)=1(\operatorname{para} \mu=r) \quad \text { e } \quad \xi_{r}^{\mu}(x)=0(\text { para } \mu \neq r) .
$$

b) Para as rotações, comparando-se (4.16) com (4.23), tem-se que $r=5, \ldots, 10$. Os parâmetros $\varepsilon^{r}$ são dados pela matriz de rotação, ou seja, $\varepsilon^{5}=m_{12}, \varepsilon^{6}=m_{13}, \varepsilon^{7}=m_{23}$, $\varepsilon^{8}=m_{14}, \varepsilon^{9}=m_{24}$ e $\varepsilon^{10}=m_{34}$, enquanto que as funções $\xi_{r}^{\mu}(x)$ são dadas pela tabela abaixo: 


\begin{tabular}{|c|c|c|c|}
\hline$\xi_{r}^{1}(x)$ & $\xi_{r}^{2}(x)$ & $\xi_{r}^{3}(x)$ & $\xi_{r}^{4}(x)$ \\
\hline$\xi_{5}^{1}(x)=\eta^{11} x^{2}$ & $\xi_{5}^{2}(x)=-\eta^{22} x^{1}$ & $\xi_{5}^{3}(x)=0$ & $\xi_{5}^{4}(x)=0$ \\
\hline$\xi_{6}^{1}(x)=\eta^{11} x^{3}$ & $\xi_{6}^{2}(x)=0$ & $\xi_{6}^{3}(x)=-\eta^{33} x^{l}$ & $\xi_{6}^{4}(x)=0$ \\
\hline$\xi_{7}^{1}(x)=0$ & $\xi_{7}^{2}(x)=\eta^{22} x^{3}$ & $\xi_{7}^{3}(x)=-\eta^{33} x^{2}$ & $\xi_{7}^{4}(x)=0$ \\
\hline$\xi_{8}^{1}(x)=\eta^{11} x^{4}$ & $\xi_{8}^{2}(x)=0$ & $\xi_{8}^{3}(x)=0$ & $\xi_{8}^{4}(x)=-\eta^{44} x^{1}$ \\
\hline$\xi_{9}^{1}(x)=0$ & $\xi_{9}^{2}(x)=\eta^{22} x^{4}$ & $\xi_{9}^{3}(x)=0$ & $\xi_{9}^{4}(x)=-\eta^{44} x^{2}$ \\
\hline$\xi_{10}^{1}(x)=0$ & $\xi_{10}^{2}(x)=0$ & $\xi_{10}^{3}(x)=\eta^{33} x^{4}$ & $\xi_{10}^{4}(x)=-\eta^{44} x^{3}$ \\
\hline
\end{tabular}

Tabela 4.1 - Funções $\xi_{r}^{\mu}(x)$ para as rotações no espaço-tempo de Minkowski.

c) Para as dilatações, comparando-se (4.17) com (4.23), tem-se que $r=11 . \mathrm{O}$ parâmetro $\varepsilon^{r}$ é dado por $\varepsilon^{l 1}=\lambda$, enquanto as funções $\xi_{r}^{\mu}(x)$ são dadas pela relação:

$$
\xi_{11}^{\mu}(x)=x^{\mu}
$$

d) Para as transformações conformes especiais, comparando-se (4.18) com (4.23), tem-se que $r=12, \ldots, 15$. Os parâmetros $\varepsilon^{r}$ são dados por $\varepsilon^{12}=s_{1}, \varepsilon^{13}=s_{2}, \varepsilon^{14}=s_{3}$, $\varepsilon^{15}=s_{4}$, enquanto as funções $\xi_{r}^{\mu}(x)$ são dadas pela relação:

$$
\xi_{r}^{\mu}(x)=2 x^{\beta} x^{\mu}-x_{v} x^{v} \eta^{\mu \beta}
$$

Deste modo, tem-se que as transformações conformes no espaço-tempo quadridimensional dependem de quinze parâmetros: quatro associados a translações, seis a rotações, um para a dilatação e quatro para as transformações conformes especiais. Como será discutido no Capítulo VI, estes parâmetros estão associados a expressões de divergência nula que representariam quinze leis de conservação para o campo eletromagnético. Essas leis estão associadas à invariância conforme das equações de Maxwell, que será discutida no próximo capítulo. 


\subsection{As transformações de Lorentz e de Poincaré.}

Nesta seção, apresentam-se as transformações de Lorentz e Poincaré seguindo-se o caminho de textos sobre a teoria da relatividade [We-72, Ri-06]. Essas transformações formam um sub-grupo das transformações conformes de coordenadas no espaço-tempo, onde o fator de escala é unitário, ou seja, $\sigma(x)=1$, e o tensor métrico $\eta_{\mu \nu}$ mantém sua forma. Neste caso, a relação (2.17) reduz-se a:

$$
\eta_{\mu \nu} \frac{\partial x^{\prime \mu}}{\partial x^{\alpha}} \frac{\partial x^{\prime}}{\partial x^{\beta}}=\eta_{\alpha \beta}
$$

Para que as transformações de coordenadas que obedecem à relação acima sejam determinadas, deve-se diferenciar primeiramente esta relação com respeito a $x^{\mathcal{E}}$. Deste modo, tem-se:

$$
\eta_{\mu \nu} \frac{\partial^{2} x^{\prime \mu}}{\partial x^{\alpha} \partial x^{\varepsilon}} \frac{\partial x^{\prime \nu}}{\partial x^{\beta}}+\eta_{\mu \nu} \frac{\partial x^{\prime \mu}}{\partial x^{\alpha}} \frac{\partial^{2} x^{\prime \nu}}{\partial x^{\beta} \partial x^{\varepsilon}}=0
$$

A seguir, trocam-se os índices $\alpha$ e $\varepsilon$, e soma-se a equação resultante à original. $\mathrm{O}$ mesmo é feito com os índices $\mathcal{E}$ e $\beta$. O resultado é, a seguir, subtraído da operação anterior, o que leva a:

$$
\begin{gathered}
\eta_{\mu \nu}\left(\frac{\partial^{2} x^{\prime \mu}}{\partial x^{\alpha} \partial x^{\varepsilon}} \frac{\partial x^{\prime \nu}}{\partial x^{\beta}}+\frac{\partial^{2} x^{\prime \prime}}{\partial x^{\beta} \partial x^{\varepsilon}} \frac{\partial x^{\prime \mu}}{\partial x^{\alpha}}+\frac{\partial^{2} x^{\prime \mu}}{\partial x^{\varepsilon} \partial x^{\alpha}} \frac{\partial x^{\prime \nu}}{\partial x^{\beta}}+\frac{\partial^{2} x^{\prime \nu}}{\partial x^{\beta} \partial x^{\alpha}} \frac{\partial x^{\prime \mu}}{\partial x^{\varepsilon}}\right. \\
\left.-\frac{\partial^{2} x^{\prime \mu}}{\partial x^{\alpha} \partial x^{\beta}} \frac{\partial x^{\prime \nu}}{\partial x^{\varepsilon}}-\frac{\partial^{2} x^{\prime \nu}}{\partial x^{\varepsilon} \partial x^{\beta}} \frac{\partial x^{\prime \mu}}{\partial x^{\alpha}}\right)=0 .
\end{gathered}
$$

Após o cancelamento de quatro dos termos da relação acima, tem-se:

$$
2 \eta_{\mu v} \frac{\partial^{2} x^{\prime \mu}}{\partial x^{\alpha} \partial x^{\varepsilon}} \frac{\partial x^{\prime \nu}}{\partial x^{\beta}}=0 .
$$


Como $\eta_{\mu v}$ e $\frac{\partial x^{\prime \nu}}{\partial x^{\beta}}$ são matrizes não singulares, obtém-se finalmente:

$$
\frac{\partial^{2} x^{\prime \mu}}{\partial x^{\alpha} \partial x^{\varepsilon}}=0
$$

A solução geral para a equação acima é do tipo:

$$
x^{\prime \mu}=\Lambda_{\beta}^{\mu} x^{\beta}+a^{\mu},
$$

onde $a^{\mu}$ e $\Lambda_{\beta}^{\mu}$ são constantes.

As transformações de coordenadas do tipo (4.27) são denominadas transformações gerais de Lorentz ou transformações de Poincaré, e formam o grupo não homogêneo de Lorentz ou grupo de Poincaré. Quando $a^{\mu}=0$, tem-se o chamado grupo homogêneo de Lorentz.

O grupo de Poincaré é, portanto, um sub-grupo das transformações conformes no espaço-tempo de Minkowski em que fator de escala é dado por $\sigma(x)=1$.

As transformações de Poincaré são relações entre elementos de uma classe especial de referenciais, chamados de referenciais inerciais. A linearidade das transformações de coordenadas para referenciais inerciais deve-se à homogeneidade e isotropia do espaço-tempo e à validade da primeira lei de Newton [Ri-06].

Uma maneira alternativa, porém mais restritiva, e usada em textos de relatividade para se verificar essa linearidade, é se partir do movimento de uma partícula livre (ausência de forças) movendo-se num referencial inercial $S$. Pela segunda lei de Newton, tem-se que $d x^{i} / d t$ é constante, onde $x^{i} \quad(i=1,2,3)$ são as coordenadas cartesianas da partícula no instante $t$. Se o tempo próprio da partícula é $\tau$, tem-se que, devido à homogeneidade do tempo, $d t / d \tau$ também é constante. Essas considerações implicam que $d x^{\mu} / d \tau$ também é constante, de modo que:

$$
\frac{d^{2} x^{\mu}}{d \tau^{2}}=0, \mu=1,2,3,4
$$


Do mesmo modo, em relação a um referencial $S^{\prime}$, com velocidade constante em relação a $S$, tem-se que:

$$
\frac{d^{2} x^{\prime \mu}}{d \tau^{2}}=0
$$

Desenvolvendo-se a relação acima, obtém-se:

$$
\frac{d^{2} x^{\prime \mu}}{d \tau^{2}}=\frac{d}{d \tau}\left(\frac{d x^{\prime \mu}}{d \tau}\right)=\frac{d}{d \tau}\left(\frac{\partial x^{\prime \mu}}{\partial x^{\nu}} \frac{d x^{\nu}}{d \tau}\right)=\frac{\partial x^{\prime \mu}}{\partial x^{\nu}} \frac{d^{2} x^{\nu}}{d \tau^{2}}+\frac{\partial^{2} x^{\prime \mu}}{\partial x^{\nu} \partial x^{\sigma}} \frac{d x^{\sigma}}{d \tau}=0
$$

A relação acima é válida para partículas livres, isto é, não submetidas à ação de forças (de interação) externas. Neste caso, a igualdade acima implica que:

$$
\frac{\partial^{2} x^{\prime \mu}}{\partial x^{\nu} \partial x^{\sigma}}=0
$$

Ou seja, a homogeneidade do espaço e do tempo e a primeira lei de Newton implicam que as transformações de coordenadas entre referenciais inerciais devem ser lineares.

A partir da linearidade da transformação seguem-se, na maioria dos textos de relatividade restrita, raciocínios análogos ao do trabalho original de Einstein [Ei-05] para se determinar as transformações de Lorentz: parte-se do postulado da relatividade na sua forma restrita (as leis que descrevem os fenômenos físicos são as mesmas em todos os sistemas de referência inerciais) e da constância da velocidade da luz (a eletrodinâmica de Maxwell é uma teoria física). Tomam-se dois sistemas de referência inerciais $S$ e $S^{\prime}$, cujas coordenadas de um evento no espaço-tempo são, respectivamente, $(x, y, z, t)$ e $\left(x^{\prime}, y^{\prime}, z^{\prime}, t^{\prime}\right)$, e considera-se a relação (4.27) com $a^{\mu}=0$, por simplicidade. Também, por simplicidade, toma-se $S$ e $S^{\prime}$ com velocidade relativa $v$ constante e no sentido do eixo $x$.

Se um sinal luminoso é emitido em $t=t^{\prime}=0$, instantes em que as origens dos sistemas, $O$ e $O^{\prime}$, coincidem, tem-se que:

$$
x^{2}+y^{2}+z^{2}=t^{2} \text { em } S \quad \text { e } \quad x^{\prime 2}+y^{\prime 2}+z^{\prime 2}=t^{\prime 2} \text { em } S^{\prime} .
$$


Pelo princípio da constância da velocidade da luz, as coordenadas da partícula e os tempos em $S$ e $S^{\prime}$ estão interligados por:

$$
x^{2}+y^{2}+z^{2}-t^{2}=x^{\prime 2}+y^{\prime 2}+z^{\prime 2}-t^{\prime 2} .
$$

Dessa igualdade e da linearidade das transformações, obtêm-se facilmente as relações da transformação de Lorentz:

$$
x^{\prime}=\gamma(v)(x-v t), \quad t^{\prime}=\gamma(v)(t-v x), \quad y^{\prime}=y \quad \text { e } \quad z^{\prime}=z .
$$

As leis da física devem ser invariantes sob essas transformações e também por translações espaciais e temporais, caso em que $a^{\mu} \neq 0$ em (4.27). Deve-se notar que a invariância se mantém para qualquer orientação de $S$ em relação a $S^{\prime}$ e, também, da velocidade relativa entre os referenciais.

É bem conhecido que, devido a essa interligação entre espaço e tempo, cenário dos eventos físicos, as leis da Mecânica tiveram que sofrer modificações para que continuassem a ser leis físicas. As conseqüências como a dependência da massa com a velocidade, relação massa-energia, e muitas outras decorrem das transformações de Lorentz. 


\section{CAPÍtTULO V}

\section{A INVARIÂNCIA CONFORME DAS EQUAÇÕES DA ELETRODINÂMICA.}

Neste capítulo, estuda-se a invariância das equações da eletrodinâmica com relação a mudanças de sistemas de referência no espaço-tempo de Minkowski. É bem conhecido que as equações da Eletrodinâmica, nesses sistemas, permanecem invariantes por transformações compostas por translações e rotações. Como exposto no Capítulo II, os trabalhos de Bateman e Cunningham de 1909 indicaram que as equações da eletrodinâmica permaneciam invariantes por um conjunto mais geral de transformações. Esses trabalhos foram apresentados cinco anos após Lorentz mostrar a invariância das equações de Maxwell pelas transformações que levam o seu nome.

$\mathrm{Na}$ primeira parte do capítulo, faz-se uma discussão sobre os sistemas de coordenadas curvilíneas no espaço-tempo plano e a formulação das equações de Maxwell em termos destas coordenadas. Na segunda parte, discute-se a classe de sistemas para os quais essas equações permanecem invariantes. Nessa discussão, utilizam-se resultados apresentados na primeira parte deste capítulo e também nos capítulos II e IV.

\subsection{As equações de Maxwell em coordenadas curvilíneas.}

Conforme visto no Capítulo III, em termos das coordenadas cartesianas $x^{\mu}$, as equações de Maxwell são dadas pelas relações (3.8) e (3.9):

$$
\varepsilon^{\mu \nu \rho \sigma} \frac{\partial F_{v \rho}}{\partial x^{\sigma}}=0 \quad \text { e } \quad \frac{\partial F^{\mu v}}{\partial x^{v}}=j^{\mu}
$$

onde $F^{\mu \nu}=-F^{v \mu}$ é o tensor do campo eletromagnético, $j^{\mu}$ é o quadrivetor densidade de corrente e $\varepsilon^{\mu v \rho \sigma}$ é o símbolo de Levi-Civita.

No que se segue, serão considerados sistemas de coordenadas curvilíneas [Di-75, Di-98, Pa-10, Ri-06, We-72] no espaço-tempo plano. Num sistema cartesiano, o tensor métrico $\eta_{\mu v}$ não depende das coordenadas e o elemento de linha fica na forma 
$d s^{2}(x)=\eta_{\mu v} d x^{\mu} d x^{v}$. Uma transformação para um sistema curvilíneo $x^{\prime}$ leva o elemento de linha para a forma $d s^{\prime 2}\left(x^{\prime}\right)=g_{\alpha \beta^{\prime}}\left(x^{\prime}\right) d x^{\prime}{ }^{\alpha} d x^{\prime} \beta$, com um tensor métrico $g_{\alpha \beta^{\prime}}\left(x^{\prime}\right)$, em geral, dependente das coordenadas. Se o espaço é plano, sempre se pode encontrar uma transformação de coordenadas de $x^{\prime}$ para $x$ onde $g_{\alpha \beta}^{\prime}\left(x^{\prime}\right)$ fica na forma $\eta_{\mu v}$.

Para se verificar sob quais transformações as equações da eletrodinâmica mantêm as formas (3.8) e (3.9), colocam-se estas equações num modo que englobem as coordenadas curvilíneas. Para isso, parte-se da equação de movimento de uma partícula livre em um sistema de referência cartesiano inercial. Nessa situação, tomando-se $\bar{\xi}^{\mu}$ como coordenadas da partícula no espaço-tempo, tem-se:

$$
\frac{d^{2} \bar{\xi}^{\mu}}{d \tau^{2}}=0
$$

onde $d \tau^{2}=-\eta_{\mu \nu} d \bar{\xi}^{\mu} d \bar{\xi}^{V}$ é o intervalo de tempo próprio da partícula.

Considerando-se, agora, a passagem de $\xi^{\mu}$ para coordenadas curvilíneas arbitrárias $x^{\mu}$, a equação acima fica na forma:

$$
\frac{d}{d \tau}\left(\frac{\partial \xi^{\mu}}{\partial x^{\alpha}} \frac{d x^{\alpha}}{d \tau}\right)=\frac{d^{2} x^{\alpha}}{d \tau^{2}} \frac{\partial \bar{\xi}^{\mu}}{\partial x^{\alpha}}+\frac{\partial^{2} \bar{\xi}^{\mu}}{\partial x^{\alpha} \partial x^{\beta}} \frac{d x^{\alpha}}{d \tau} \frac{d x^{\beta}}{d \tau}=0
$$

Multiplicando-se a relação anterior por $\frac{\partial x^{\lambda}}{\partial \bar{\xi}^{\mu}}$ e considerando-se que $\frac{\partial \xi^{\mu}}{\partial x^{\alpha}} \frac{\partial x^{\lambda}}{\partial \bar{\xi}^{\mu}}=\delta_{\alpha}^{\lambda}$, tem-se que, em relação ao sistema $x^{\mu}$, o movimento da partícula é dado por:

$$
\frac{d^{2} x^{\lambda}}{d \tau^{2}}+\Gamma_{\alpha \beta}^{\lambda} \frac{d x^{\alpha}}{d \tau} \frac{d x^{\beta}}{d \tau}=0
$$

onde o símbolo $\Gamma_{\alpha \beta}^{\lambda}$ é chamado conexão afim e é dado pela expressão: 


$$
\Gamma_{\alpha \beta}^{\lambda}=\frac{\partial x^{\lambda}}{\partial \xi^{\mu}} \frac{\partial^{2} \bar{\xi}^{\mu}}{\partial x^{\alpha} \partial x^{\beta}}
$$

A conexão afim também pode ser dada em função do tensor métrico $g_{\alpha \beta^{(x)}}$ relativo às coordenadas $x^{\mu}$. Para isso, toma-se a relação entre $g_{\alpha \beta}(x)$ e o tensor métrico $\eta_{\mu \nu}$ no sistema $\bar{\xi}^{\mu}$, dada por:

$$
g_{\alpha \beta}(x)=\frac{\partial \bar{\xi}^{\mu}}{\partial x^{\alpha}} \frac{\partial \bar{\xi}^{\nu}}{\partial x^{\beta}} \eta_{\mu v}
$$

Diferenciando-se essa expressão em relação à coordenada $x^{\lambda}$, tem-se:

$$
\frac{\partial g_{\alpha \beta}}{\partial x^{\lambda}}=\frac{\partial^{2} \bar{\xi}^{\mu}}{\partial x^{\lambda} \partial x^{\alpha}} \frac{\partial \xi^{v}}{\partial x^{\beta}} \eta_{\mu v}+\frac{\partial \xi^{\mu}}{\partial x^{\alpha}} \frac{\partial^{2} \bar{\xi}^{v}}{\partial x^{\lambda} \partial x^{\beta}} \eta_{\mu v} .
$$

Multiplicando-se, agora, a relação (5.1) por $\frac{\partial \bar{\xi}^{\mu}}{\partial x^{\lambda}}$, tem-se que:

$$
\Gamma_{\alpha \beta}^{\lambda} \frac{\partial \bar{\xi}^{\mu}}{\partial x^{\lambda}}=\frac{\partial^{2} \bar{\xi}^{\mu}}{\partial x^{\alpha} \partial x^{\beta}}
$$

Substituindo-se a relação acima na expressão (5.3), obtém-se:

$$
\frac{\partial g_{\alpha \beta}}{\partial x^{\lambda}}=\Gamma_{\lambda \alpha}^{\rho} \frac{\partial \bar{\xi}^{\mu}}{\partial x^{\rho}} \frac{\partial \xi^{v}}{\partial x^{\beta}} \eta_{\mu \nu}+\Gamma_{\lambda \beta}^{\rho} \frac{\partial \xi^{v}}{\partial x^{\rho}} \frac{\partial \xi^{\mu}}{\partial x^{\alpha}} \eta_{\mu \nu}
$$

Utilizando-se (5.2) na relação acima, tem-se que:

$$
\frac{\partial g_{\alpha \beta}}{\partial x^{\lambda}}=\Gamma_{\lambda \alpha}^{\rho} g_{\rho \beta}+\Gamma_{\lambda \beta}^{\rho} g_{\rho \alpha}
$$


Somando-se essa expressão com ela mesma, mas com o índice $\alpha$ trocado por $\lambda$, e subtraindo-se o resultado pela mesma relação $\operatorname{com} \beta$ trocado por $\lambda$, obtém-se:

$$
\begin{aligned}
\frac{\partial g_{\alpha \beta}}{\partial x^{\lambda}}+\frac{\partial g_{\lambda \beta}}{\partial x^{\alpha}}-\frac{\partial g_{\alpha \lambda}}{\partial x^{\beta}} & =g_{\rho \beta} \Gamma_{\lambda \alpha}^{\rho}+g_{\rho \alpha} \Gamma_{\lambda \beta}^{\rho}+g_{\rho \beta} \Gamma_{\alpha \lambda}^{\rho}+g_{\rho \lambda} \Gamma_{\alpha \beta}^{\rho}- \\
& -g_{\rho \lambda} \Gamma_{\beta \alpha}^{\rho}-g_{\rho \alpha} \Gamma_{\beta \lambda}^{\rho} .
\end{aligned}
$$

Como $\Gamma_{\alpha \beta}^{\rho}$ e $g_{\alpha \beta}$ são simétricos em relação aos índices $\alpha$ e $\beta$, essa expressão toma a forma:

$$
\frac{\partial g_{\alpha \beta}}{\partial x^{\lambda}}+\frac{\partial g_{\lambda \beta}}{\partial x^{\alpha}}-\frac{\partial g_{\alpha \lambda}}{\partial x^{\beta}}=2 g_{\rho \beta} \Gamma_{\lambda \alpha}^{\rho}
$$

Multiplicando-se a expressão acima pela matriz inversa ${ }_{g}^{\beta \sigma}$, onde $g^{\beta \sigma} g_{\rho \beta}=\delta_{\rho}^{\sigma}$, obtém-se $\Gamma_{\lambda \alpha}^{\sigma}$ em função do tensor métrico na forma:

$$
\Gamma_{\lambda \alpha}^{\sigma}=\frac{1}{2} g \beta \sigma\left(\frac{\partial g_{\alpha \beta}}{\partial x^{\lambda}}+\frac{\partial g \lambda \beta}{\partial x^{\alpha}}-\frac{\partial g_{\alpha \lambda}}{\partial x^{\beta}}\right) .
$$

$\Gamma_{\lambda \alpha}^{\sigma}$, quando colocado em função do tensor métrico, é muitas vezes representado por $\left\{\begin{array}{c}\sigma \\ \lambda \alpha\end{array}\right\}$ e chamado de símbolo de Christoffel.

Em particular, se o sistema de coordenadas $x^{\mu}$ é cartesiano, tem-se que $g_{\mu v}=\eta_{\mu v} \mathrm{e}$ o símbolo de Christoffel se anula, de modo que a equação de movimento da partícula livre fica na forma $\frac{d^{2} x^{\mu}}{d \tau^{2}}=0$ inicialmente vista.

Verifica-se, a seguir, como a conexão afim (5.1) se transforma quando se passa do sistema de coordenadas $x^{\mu}$ para outro sistema de coordenadas $x^{\prime}{ }^{\mu}$. Neste caso, tem-se que: 


$$
\begin{gathered}
\Gamma_{\alpha \beta}^{\prime \lambda}=\frac{\partial x^{\prime \lambda}}{\partial \bar{\xi}^{\mu}} \frac{\partial^{2} \bar{\xi}^{\mu}}{\partial x^{\prime} \alpha \partial x^{\prime \beta}} \Rightarrow \\
\Gamma_{\alpha \beta}^{\prime \lambda}=\frac{\partial x^{\prime \lambda}}{\partial x^{\rho}} \frac{\partial x}{\partial \bar{\xi}^{\mu}}\left[\frac{\partial x^{\sigma}}{\partial x^{\prime} \beta} \frac{\partial x^{\tau}}{\partial x^{\prime} \alpha} \frac{\partial^{2} \bar{\xi}^{\mu}}{\partial x^{\tau} \partial x^{\sigma}}+\frac{\partial^{2} x^{\sigma}}{\partial x^{\prime \alpha} \partial x^{\prime} \beta} \frac{\partial \bar{\xi}^{\mu}}{\partial x^{\sigma}}\right] .
\end{gathered}
$$

Considerando-se que $\delta_{\sigma}^{\rho}=\frac{\partial x^{\rho}}{\partial \bar{\xi}^{\mu}} \frac{\partial \bar{\xi}^{\mu}}{\partial x^{\sigma}}$ e utilizando-se a relação (5.1) para $\Gamma_{\tau \sigma}^{\rho}$ na expressão acima, obtém-se:

$$
\Gamma_{\alpha \beta}^{\prime \lambda}=\frac{\partial x^{\prime} \lambda}{\partial x^{\rho}} \frac{\partial x^{\tau}}{\partial x^{\prime} \alpha} \frac{\partial x^{\sigma}}{\partial x^{\prime} \beta} \Gamma_{\tau \sigma^{\prime}}^{\rho}+\frac{\partial x^{\prime} \lambda}{\partial x^{\rho}} \frac{\partial^{2} x^{\rho}}{\partial x^{\prime} \alpha \partial x^{\prime} \beta}
$$

Diferenciando-se $\frac{\partial x^{\prime} \lambda}{\partial x^{\rho}} \frac{\partial x^{\rho}}{\partial x^{\prime} \beta}=\delta_{\beta}^{\lambda}$ com respeito a $x^{\prime \alpha}$ tem-se:

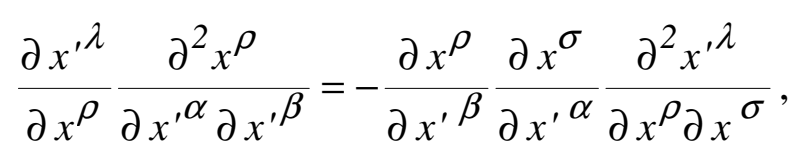

que, substituída em (5.4), leva à seguinte relação de transformação para $\Gamma_{\tau \sigma}^{\rho}$ :

$$
\Gamma_{\alpha \beta}^{\prime \lambda}=\frac{\partial x^{\prime} \lambda}{\partial x^{\rho}} \frac{\partial x^{\tau}}{\partial x^{\prime} \alpha} \frac{\partial x^{\sigma}}{\partial x^{\prime} \beta} \Gamma_{\tau \sigma^{-}}^{\rho}-\frac{\partial x^{\rho}}{\partial x^{\prime} \beta} \frac{\partial x^{\sigma}}{\partial x^{\prime} \alpha} \frac{\partial^{2} x^{\prime} \lambda}{\partial x^{\rho} \partial x^{\sigma}}
$$

O primeiro termo do lado direito da equação (5.5) está de acordo com a lei de transformação de um tensor de ordem três, uma vez contravariante e duas, covariante. Entretanto, o segundo termo não se transforma como um tensor. Portanto, a conexão afim não é um tensor.

No entanto, pode-se utilizar a expressão acima para se obter uma grandeza que se transforme como um tensor. Para isso, parte-se da transformação de um vetor $V^{\alpha}$ do sistema $x^{\mu}$ para $x^{\prime \mu}$, ou seja: 


$$
V^{\prime \alpha}=\frac{\partial x^{\prime \alpha}}{\partial x^{\beta}} V^{\beta}
$$

Diferenciando-se $V^{\prime \alpha}$ em relação a $x^{\prime \lambda}$, obtém-se:

$$
\frac{\partial V^{\prime \alpha}}{\partial x^{\prime \lambda}}=\frac{\partial x^{\prime} \alpha}{\partial x^{\beta}} \frac{\partial x^{\rho}}{\partial x^{\prime} \lambda} \frac{\partial V^{\beta}}{\partial x^{\rho}}+\frac{\partial^{2} x^{\prime \alpha}}{\partial x^{\beta} \partial x^{\rho}} \frac{\partial x^{\rho}}{\partial x^{\prime} \lambda} V^{\beta} .
$$

Verifica-se que o primeiro termo do lado direito da equação (5.7) transforma a derivada parcial de $V^{\beta}$ em relação a $x^{\rho}$ da mesma forma que um tensor misto, mas o segundo termo indica que $\frac{\partial V^{\beta}}{\partial x^{\rho}}$ não é um tensor. Entretanto, pode-se utilizar (5.7) para encontrar uma forma da derivada de $V^{\beta}$ que se transforme como um tensor. Para isso, inicialmente multiplica-se (5.5) por (5.6):

$$
\Gamma_{\lambda \kappa^{\prime} V^{\prime}}^{\alpha}=\frac{\partial x^{\prime} \alpha}{\partial x^{\beta}} \frac{\partial x^{\rho}}{\partial x^{\prime} \lambda} \Gamma_{\rho \sigma}^{\beta} V^{\sigma}-\frac{\partial^{2} x^{\prime \alpha}}{\partial x^{\rho} \partial x^{\sigma}} \frac{\partial x^{\rho}}{\partial x^{\prime}{ }^{\lambda}} V^{\sigma}
$$

e, em seguida, deve-se somar esse resultado com (5.7). Essas operações levam à seguinte relação:

$$
\frac{\partial V^{\prime \alpha}}{\partial x^{\prime \lambda}}+\Gamma_{\lambda \kappa^{\prime}}^{\alpha} V^{\prime \kappa}=\frac{\partial x^{\prime \alpha}}{\partial x^{\beta}} \frac{\partial x^{\rho}}{\partial x^{\prime} \lambda}\left(\frac{\partial V^{\beta}}{\partial x^{\rho}}+\Gamma_{\rho \sigma}^{\beta} V^{\sigma}\right)
$$

Esse resultado é importante, pois indica claramente que a grandeza $\frac{\partial V^{\beta}}{\partial x^{\rho}}+\Gamma{ }_{\rho \sigma}^{\beta} V^{\sigma}$ se transforma como um tensor. Define-se, então, a derivada covariante de $V^{\alpha}$ pela relação:

$$
V_{; \lambda}^{\alpha}=\frac{\partial V^{\alpha}}{\partial x^{\lambda}}+\Gamma_{\lambda \kappa}^{\alpha} V^{\kappa}
$$


De (5.8), tem-se que a derivada covariante de $V^{\alpha}$ transforma-se como um tensor, de modo que ela mantém sua forma em todos os sistemas de coordenadas.

Em particular, num sistema de coordenadas cartesianas $x^{\mu}, \Gamma_{\lambda \kappa}^{\alpha}=0$ e a derivada covariante se reduz a uma derivada comum.

Usando-se a mesma linha de raciocínio, podem-se obter expressões para derivadas covariantes de tensores de ordem dois ou superiores [Pa-10, We-72]. Por exemplo, para o tensor contravariante de segunda ordem $T^{\mu \nu}$, a derivada covariante em relação a $x^{\kappa}$ é dada por:

$$
T^{\mu \nu}{ }_{{ }_{\kappa}}=\frac{\partial T^{\mu \nu}}{\partial x^{\kappa}}+\Gamma_{\kappa \lambda}^{\mu} T^{\lambda v}+\Gamma_{\kappa \lambda}^{v} T^{\mu \lambda}
$$

e a divergência covariante de $T^{\mu \nu}$ é dada por:

$$
T_{{ }_{v}}^{\mu v}=\frac{\partial T^{\mu v}}{\partial x^{v}}+\Gamma_{\lambda v^{\mu}}^{\mu} T^{\lambda v}+\Gamma_{v \lambda}^{v} T^{\mu \lambda}
$$

Usando-se, agora, o resultado [We-72]:

$$
\Gamma_{v \lambda}^{v}=\frac{1}{\sqrt{|g|}} \frac{\partial}{\partial x^{\lambda}} \sqrt{|g|}
$$

em (5.9), tem-se que:

$$
T_{; v}^{\mu v}=\frac{1}{\sqrt{|g|}} \frac{\partial\left(\sqrt{|g|} T^{\mu v}\right)}{\partial x^{v}}+\Gamma_{\lambda v}^{\mu} T^{\lambda v}
$$

Em particular, para $T^{\mu \nu}$ anti-simétrico, o segundo termo do lado direito da expressão acima se anula, de forma que: 


$$
T_{{ }_{\nu}}^{\mu \nu}=\frac{1}{\sqrt{|g|}} \frac{\partial\left(\sqrt{|g|} T^{\mu v}\right)}{\partial x^{v}} .
$$

Pode-se também mostrar que, para um tensor covariante de segunda ordem $T_{\mu v}$, a derivada covariante é dada por:

$$
T_{\mu \nu ; \lambda}=\frac{\partial T_{\mu \nu}}{\partial x^{\lambda}}-\Gamma_{\mu \lambda}^{\rho} T_{\rho v}-\Gamma_{v \lambda}^{\rho} T_{\mu \rho} .
$$

Se $T_{\mu \nu}$ é anti-simétrico, e devido à simetria de $\Gamma_{\mu \lambda}^{\rho}$ em relação aos dois índices inferiores $\mu$ e $\lambda$, tem-se que a soma tripla de $T_{\mu v ; \lambda}$ com os índices permutados ciclicamente é dada por:

$$
T_{\mu \nu ; \lambda}+T_{\lambda \mu ; \nu^{\prime}}+T_{v \lambda ; \mu}=T_{\mu \nu, \lambda}+T_{\lambda \mu, v}+T_{\nu \lambda, \mu}
$$

pois todos os termos que contêm símbolos de Christoffel se cancelam.

Tendo em mente os resultados apresentados até aqui e colocando as equações de Maxwell numa forma covariante, mas agora incluindo sistemas curvilíneos, tem-se que essas equações escrevem-se como:

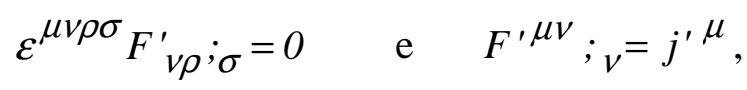

que, para sistemas cartesianos, em virtude de $\Gamma_{\mu \lambda}^{\rho}=0$, reproduzem as equações (3.8) e (3.9).

Estas equações mantêm sua forma por transformações de Lorentz-Poincaré, ou seja, existe uma classe de sistemas de referência cujas coordenadas se relacionam por essas transformações e que preservam a forma dessas equações. Fica, entretanto, a questão de se saber se existem outras transformações para as quais as formas (3.8) e (3.9) se mantêm. Para tentar responder a essa questão, considera-se a anti-simetria do tensor eletromagnético nas 
formas $F^{\mu v}$ e $F_{\mu \nu}$ e utilizam-se as relações (5.10) e (5.11). Nestas condições, as equações (5.12) ficam nas formas:

$$
\varepsilon^{\mu v \rho \sigma} \frac{\partial F^{\prime} v \rho}{\partial x^{\prime} \sigma}=0
$$

$\mathrm{e}$

$$
\frac{\partial\left(\sqrt{\left|g^{\prime}\right|} F^{\prime} \mu \nu\right)}{\partial x^{\prime \nu}}=\sqrt{\left|g^{\prime}\right|} j^{\prime \mu} .
$$

Comparando essas duas últimas equações, respectivamente, com (3.8) e (3.9), vê-se que a equação (5.13) preserva sua forma quando se passa de coordenadas cartesianas para curvilíneas gerais. $\mathrm{O}$ mesmo não acontece com as equações não homogêneas de Maxwell, o que pode ser visto comparando-se (3.9) com (5.14).

Para se examinar essa questão, deve-se retomar a relação (2.15), que envolve o módulo do Jacobiano de transformações entre sistemas de referência $x$ e $x^{\prime}$ :

$$
\left|J^{\prime}\right|=\left|\operatorname{det}\left[\frac{\partial x^{\prime} \alpha}{\partial x^{\mu}}\right]\right|=\sqrt{\frac{|g|}{\left|g^{\prime}\right|}} .
$$

Considerando-se, agora, a transformação de coordenadas cartesianas $x^{\mu}$ no espaço de Minkowski, onde $g_{\mu v}=\eta_{\mu v}(|g|=1)$, para coordenadas curvilíneas $x^{\prime \mu}$, tem-se que a relação acima fica:

$$
\sqrt{\left|g^{\prime}\right|}=\left|J^{\prime}\right|^{-1}
$$

que inserida em (5.14) leva a:

$$
\frac{\partial\left(\left|J^{\prime}\right|^{-1} F^{\prime} \mu \nu\right)}{\partial x^{\prime \nu}}=\left|J^{\prime}\right|^{-1} j^{\prime \mu} .
$$


Deste modo, considerando-se a relação acima para a equação não homogênea e que (5.13) não depende do Jacobiano $J^{\prime}$, pode-se, finalmente, colocar as equações de Maxwell em coordenadas curvilíneas nas formas:

$$
\varepsilon^{\mu v \rho \sigma} \frac{\partial F^{\prime} v \rho}{\partial x^{\prime} \sigma}=0
$$

$\mathrm{e}$

$$
\frac{\partial \widetilde{F}^{\prime \mu \nu}}{\partial x^{\prime \nu}}=\tilde{j}^{\prime \mu}
$$

onde:

$$
\begin{gathered}
F^{\prime} \mu \nu=\left(\frac{\partial x^{\rho}}{\partial x^{\prime \mu}}\right)\left(\frac{\partial x^{\sigma}}{\partial x^{\prime \nu}}\right) F_{\rho \sigma}, \\
\widetilde{F}^{, \mu \nu}=\left|J^{\prime}\right|^{-1} F^{, \mu \nu}=\left|J^{\prime}\right|^{-1}\left(\frac{\partial x^{\prime} \mu}{\partial x^{\rho}}\right)\left(\frac{\partial x^{\prime \nu}}{\partial x^{\sigma}}\right) F^{\rho \sigma},
\end{gathered}
$$

$\mathrm{e}$

$$
\tilde{j}^{\prime} \mu=\left|J^{\prime}\right|^{-1} j^{\prime \mu}=\left|J^{\prime}\right|^{-1}\left(\frac{\partial x^{\prime \mu}}{\partial x^{\rho}}\right) j^{\rho} .
$$

\subsection{A invariância conforme das equações de Maxwell.}

A partir das formas (5.13) e (5.15) das equações de Maxwell em coordenadas curvilíneas, examina-se agora qual a condição que deve ser imposta a uma transformação no espaço-tempo para que as formas (3.8) e (3.9) sejam preservadas.

Primeiramente, como já foi observado, (5.13) mantém sua forma em todos os sistemas curvilíneos, ou seja, (5.13) é invariante para a transformação tensorial usual (5.16) de $F_{\rho \sigma}$. Entretanto, o mesmo não acontece com a equação não homogênea, pois o tensor $F^{\rho \sigma}$ não se transforma da maneira usual, como pode ser visto por (5.17).

A condição a ser imposta para a manutenção da forma cartesiana da equação não homogênea é dada por [Ro-72]:

$$
\widetilde{F}^{\prime \mu \nu}=F^{\prime \mu \nu} \text {. }
$$


Utiliza-se, agora, a condição acima e o fato que $\widetilde{F}$ e $F$ são anti-simétricos. Considerando-se também a transformação (5.17) para $\widetilde{F}^{\prime \mu \nu}$ e a transformação para o tensor eletromagnético $F^{\rho \sigma}$ em termos das coordenadas covariantes $x_{\mu}$, ou seja:

$$
F^{, \mu v}=\frac{\partial x_{\rho}}{\partial x_{\mu}^{\prime}} \frac{\partial x_{\sigma}}{\partial x_{v}^{\prime}} F^{\rho \sigma}
$$

obtém-se:

$$
\left|J^{\prime}\right|^{-1}\left(\frac{\partial x^{\prime \mu}}{\partial x^{\rho}} \frac{\partial x^{\prime \nu}}{\partial x^{\sigma}}-\frac{\partial x^{\prime \mu}}{\partial x^{\sigma}} \frac{\partial x^{\prime v}}{\partial x^{\rho}}\right)=\left(\frac{\partial x_{\rho}}{\partial x_{\mu}^{\prime}} \frac{\partial x_{\sigma}}{\partial x_{\nu}^{\prime}}-\frac{\partial x_{\rho}}{\partial x_{\nu}^{\prime}} \frac{\partial x_{\sigma}}{\partial x^{\prime} \mu}\right)
$$

que é a condição para que as equações de Maxwell se mantenham invariantes.

$$
\begin{gathered}
\text { Considerando-se que } \frac{\partial x_{\mu}^{\prime} \mu}{\partial x_{\lambda}} \frac{\partial x_{\rho}}{\partial x^{\prime} \mu}=\delta_{\rho}^{\lambda} \text {, a condição acima é equivalente à relação: } \\
\theta_{\rho}{ }^{\lambda} \theta_{\sigma}{ }^{\kappa}-\theta_{\sigma}{ }^{\lambda} \theta_{\rho}{ }^{\kappa}=\delta_{\rho}{ }^{\lambda} \delta_{\sigma}{ }^{\kappa}-\delta_{\sigma}{ }^{\lambda} \delta_{\rho}{ }^{\kappa}
\end{gathered}
$$

onde:

$$
\theta_{\rho}^{\lambda}=\frac{1}{\sqrt{\left|J^{\prime}\right|}}\left(\frac{\partial x^{\prime} \mu}{\partial x_{\lambda}}\right)\left(\frac{\partial x^{\prime} \mu}{\partial x^{\rho}}\right)
$$

Mas a condição (5.18) requer que:

$$
\theta_{\rho}^{\lambda}=\delta_{\rho}^{\lambda}
$$

de onde, finalmente, se chega à condição que deve ser satisfeita pelas possíveis formas de transformação de coordenadas que preservem as equações de Maxwell na forma (3.8) e (3.9). Essa condição é dada por: 


$$
\left(\frac{\partial x^{\prime} \mu}{\partial x_{\lambda}}\right)\left(\frac{\partial x^{\prime} \mu}{\partial x^{\rho}}\right)=\sqrt{\left|J^{\prime}\right|} \delta_{\rho}^{\lambda} .
$$

Como se verificou no Capítulo II, as transformações conformes no espaço-tempo satisfazem a equação (2.21), que é exatamente igual à condição acima e relaciona as coordenadas curvilíneas $x^{\prime \mu}$ com as cartesianas $x^{V}$ em termos do Jacobiano $J^{\prime}$ da transformação conforme.

Neste ponto, pode-se voltar ao Capítulo IV, onde se parte de (2.17) e chega-se às transformações conformes em espaços planos, ou seja, às transformações (4.19-4.22):

1) Translações (4.19):

2) Rotações (4.20):

3) Dilatações (4.21):

4) Transformações conformes especiais (4.22): $\quad x^{\prime} \mu=\frac{x^{\mu}-(x . x) s^{\mu}}{1-2(s . x)+(s . s)(x . x)}$.

Esse conjunto de transformações no espaço-tempo de Minkowski $(n=4)$ preserva as equações de Maxwell nas formas (3.8) e (3.9). Vê-se que as duas primeiras representam as transformações de Lorentz-Poincaré e as duas últimas são as transformações de escala e as transformações conformes especiais.

Com esse resultado, passa-se a estudar, no capítulo que se segue e com a ajuda do Teorema de Noether, as leis de conservação que essas transformações implicam. 


\section{CAPÍTULO VI}

\section{LEIS DE CONSERVAÇÃO ASSOCIADAS À INVARIÂNCIA CONFORME DO CAMPO ELETROMAGNÉTICO.}

Neste capítulo são obtidas, via teorema de Noether, as leis de conservação associadas à invariância conforme do campo eletromagnético. Como visto, essas invariâncias são devidas a simetrias por translações, rotações, dilatações e transformações conformes especiais no espaço-tempo. Como exposto na síntese histórica do Capítulo II, essas leis de conservação foram obtidas pela primeira vez em 1921 por Bessel-Hagen [Be-21] como uma aplicação do teorema de Noether para a invariância conforme das equações de Maxwell. No estudo que se apresenta neste capítulo [Al-06, Pl-74], retomam-se os resultados apresentados no Capítulo IV, item 4.4, onde se chegou às transformações conformes no espaço-tempo, com um total de $n+1+\frac{n(n-1)}{2}+n=15$ (para $\left.n=4\right)$ parâmetros.

Inicialmente, serão discutidas as leis de conservação associadas ao momento linear, à energia e ao momento angular associados ao campo eletromagnético. Examinam-se, também, aspectos pouco conhecidos e ligados ao movimento do centro de energia do campo eletromagnético. Examinam-se, também, possíveis interpretações físicas para a transformação de dilatação e para as transformações conformes especiais, sob o ponto de vista das leis de conservação decorrentes dessas transformações.

\subsection{Leis de conservação associadas às translações no espaço-tempo.}

Nesta seção, consideram-se as leis de conservação para o campo eletromagnético decorrentes das simetrias por translações no espaço-tempo, que são dadas pela relação (4.13):

$$
x^{\prime \mu}=x^{\mu}+a^{\mu}, \mu=1,2,3,4 .
$$

As transformações infinitesimais acima são dadas em termos dos quatro parâmetros $\varepsilon^{r}<<1(r=1,2,3,4), \varepsilon^{1}=a^{1}, \varepsilon^{2}=a^{2}, \varepsilon^{3}=a^{3}$ e $\varepsilon^{4}=a^{4}$, e das funções $\xi_{r}^{\mu}(x)$ (4.24): 


$$
\xi_{r}^{\mu}(x)=1(\text { para } \mu=r) \quad \text { e } \quad \xi_{r}^{\mu}(x)=0(\text { para } \mu \neq r) .
$$

Substituindo-se as funções $\xi_{r}^{\mu}(x)$ acima na equação de continuidade (3.37), obtémse:

$$
\frac{\partial \Theta^{\mu}}{\partial x^{\mu}}=0 ; \mu, \sigma=1,2,3,4
$$

Como, pela relação (3.36), $\Theta^{\mu}{ }_{\sigma}=\Theta^{\mu \sigma}(\sigma=1,2,3)$ e $\Theta^{\mu}{ }_{\sigma}=-\Theta^{\mu \sigma}(\sigma=4)$, a expressão acima pode ser escrita em função do tensor de energia-momento na forma $\Theta^{\mu \sigma}$, ou seja:

$$
\frac{\partial \Theta^{\mu \sigma}}{\partial x^{\mu}}=0
$$

Para se verificarem as leis de conservação associadas à equação acima, considera-se o tensor de energia-momento $\Theta^{\mu \sigma}$ explicitamente, ou seja, em termos dos campos eletromagnéticos $\vec{E}$ e $\vec{B}$ [Fr-05, Gr-96, Gr-99, Ja-99, He-95]:

$$
\Theta^{\mu \sigma}=-\frac{1}{4} \eta^{\mu \sigma} F^{\alpha \beta} F_{\alpha \beta}-F^{\mu v} F_{v}{ }^{\alpha}=\left\{\begin{array}{l}
\Theta^{44}=\frac{\left(E^{2}+B^{2}\right)}{2}=u \\
\Theta^{4 i}=\Theta^{i 4}=(\vec{E} x \vec{B})_{i}=\vec{S}^{i}=g^{i} \\
\Theta^{i j}=\Theta j i=-\left[E_{i} E_{j}+B_{i} B_{j}-\frac{1}{2} \delta_{j}^{i}\left(E^{2}+B^{2}\right)\right]=-T^{i j}
\end{array},\right.
$$

onde: $i=j=1,2,3$.

A componente $\Theta^{44}$ do tensor de energia-momento é a densidade de energia $u$ armazenada no campo eletromagnético. A componente $\Theta^{4 i}$ representa a $i$-ésima componente da densidade do momento linear $\left(g^{i}\right)$ do campo, que, em unidades naturais, é igual à $i$-ésima 
componente do vetor de Poynting $\left(S^{i}\right)$. As componentes restantes, $\Theta^{i j}$ e $\Theta^{j i}$, são dadas pelo valor negativo do tensor das tensões de Maxwell $T^{i j}$, também expresso na forma $\vec{T}$.

Em termos matriciais, o tensor $\Theta^{\mu \sigma}$ é representado na forma:

$$
\Theta^{\mu \sigma}=\left(\begin{array}{cccc}
-T^{11} & -T^{12} & -T^{13} & g^{1} \\
-T^{21} & -T^{22} & -T^{23} & g^{2} \\
-T^{31} & -T^{32} & -T^{33} & g^{3} \\
g^{1} & g^{2} & g^{3} & u
\end{array}\right)=\left(\begin{array}{cc}
-T^{i j} & g^{i} \\
g^{i} & u
\end{array}\right) .
$$

Como pode se verificar abaixo, esse tensor tem a propriedade de possuir traço nulo, ou seja:

$$
\Theta^{\mu \mu}=\Theta^{11}+\Theta^{22}+\Theta^{33}+\Theta^{44}=-T^{11}-T^{22}-T^{33}+u=0
$$

Considerando-se $\Theta^{\mu \sigma}$, tem-se que as componentes espaciais $(\sigma=1,2,3)$ de $(6.1)$ implicam na seguinte lei de conservação para o campo eletromagnético:

$$
\frac{\partial g^{i}}{\partial t}+\frac{\partial\left(-T^{i j}\right)}{\partial x^{j}}=0
$$

A relação acima é uma equação de continuidade que exprime a conservação do momento linear do campo eletromagnético, onde o tensor $-T^{i j}$ é a densidade de fluxo de momento. Na sua forma integral, essa relação fica:

$$
\frac{d}{d t} \int_{V} g^{i} d v+\oint_{S}\left(-T^{i j}\right) n_{j} d a=0
$$

onde $\int_{V} g^{i} d V$ é a $i$-ésima componente do momento total armazenado no campo eletromagnético e $n_{j}$ é a componente $j$ da normal externa a uma dada superfície fechada $S$ 
que limita o volume $V$. O termo $-T^{i j} n_{j}$ da integral de superfície acima representa a $i$-ésima componente do fluxo do momento através da superfície, ou seja, é a pressão na superfície $S$ devido ao campo.

Quando o volume $V$ passa a ser todo o espaço tridimensional, e se os campos eletromagnéticos decaem de forma suficientemente rápida a zero, o termo $\oint_{S}\left(-T^{i j}\right) n_{j} d s$ se anula. Neste caso, tem-se que as cargas de Noether (3.38) são dadas por $P^{i}=\int_{V} d^{3} x g^{i}$, para $i=1,2,3$, e expressam a conservação das três componentes do momento linear total do campo.

Considerando-se, agora, a componente temporal $(\sigma=4)$ de $(6.1)$, a seguinte lei de conservação é obtida:

$$
\frac{\partial u}{\partial t}+\vec{\nabla} \cdot \vec{S}=0
$$

A relação acima expressa o teorema de Poynting para o campo livre, ou seja, exprime a conservação da energia do campo eletromagnético, onde o vetor de Poynting $\vec{S}$ é a densidade do fluxo de energia na direção perpendicular aos campos $\vec{E}$ e $\vec{B}$. Na sua forma integral esse teorema se escreve como:

$$
\frac{d}{d t} \int_{V} u d V+\oint_{S} \vec{S} \cdot \vec{n} d a=0
$$

onde $\int_{V} u d V$ é a energia armazenada no campo eletromagnético e $\vec{n}$ é a normal à superfície $S$ que engloba o volume $V$.

Quando se considera que os campos caem de forma suficientemente rápida com as distâncias e o volume $V$ engloba todo o espaço, a equação acima expressa a conservação da energia total, onde a quantidade conservada (3.38) é dada por $P^{4}=\int_{V} d^{3} x u$.

Conclui-se, então, que as leis de conservação da energia e do momento linear do campo eletromagnético são decorrentes das simetrias por translações no espaço-tempo. 


\subsection{Leis de conservação associadas às rotações no espaço-tempo.}

Para se determinarem as leis de conservação para o campo eletromagnético associadas às rotações no espaço-tempo, considera-se a relação (4.16):

$$
x^{\prime \mu}=\left(\delta_{v}^{\mu}+\eta^{\mu \lambda} m_{\lambda v}\right) x^{\nu}, \mu=1,2,3,4 .
$$

As transformações acima são dadas em termos dos seis parâmetros $\varepsilon^{r}<<1$ $(r=5, \ldots 10)$ e das funções $\xi_{r}^{\mu}(x)$. Os parâmetros são dados por: $\varepsilon^{5}=m_{12}, \varepsilon^{6}=m_{13}$, $\varepsilon^{7}=m_{23}, \varepsilon^{8}=m_{14}, \varepsilon^{9}=m_{24}$ e $\varepsilon^{10}=m_{34}$. As funções $\xi_{r}^{\mu}(x)$ estão na Tabela 4.1. A substituição destas em (3.37) leva à equação:

$$
\frac{\partial\left(x^{\beta} \Theta^{\mu \rho}-x^{\rho} \Theta^{\mu \beta}\right)}{\partial x^{\mu}}=0
$$

$\operatorname{com}(\beta, \rho) \in\{(2,1),(3,1),(3,2),(4,1),(4,2),(4,3)\}$.

Desenvolvendo-se essa equação, tem-se que:

$$
x^{\beta} \frac{\partial \Theta^{\mu \rho}}{x^{\mu}}-x^{\rho} \frac{\partial \Theta^{\mu \beta}}{\partial x^{\mu}}+\left(\Theta^{\beta \rho}-\Theta^{\rho \beta}\right)=0 .
$$

Os dois primeiros termos da expressão acima se anulam devido a (6.1), obtida para translações. O último termo se anula devido à simetria do tensor $\Theta^{\beta \rho}$, e será visto que essa propriedade está relacionada com a conservação do momento angular do campo eletromagnético.

A equação (6.3) provém diretamente do teorema de Noether, e deste modo pode-se definir um tensor constante e de ordem três, $M^{\mu \beta \rho}$, dado por:

$$
M^{\mu \beta \rho}=x^{\beta} \Theta^{\mu \rho}-x^{\rho} \Theta^{\mu \beta}
$$


Assim, a lei de conservação (6.3) fica na forma $\frac{\partial M^{\mu \beta \rho}}{\partial x^{\mu}}=0$. Tomando-se as componentes espaciais, $(\beta, \rho)=\{(2,1),(3,1),(3,2)\}$, chega-se à equação de continuidade:

$$
\frac{\partial(\vec{r} \times \vec{g})}{\partial t}+\vec{\nabla} \cdot(\vec{T} x \vec{r})=0
$$

onde $\vec{r}$ é o vetor posição, $(\vec{r} \times \vec{g})$ é a densidade do momento angular e $(\vec{T} \times \vec{r})$ é o fluxo do momento angular associado ao campo eletromagnético.

$\mathrm{Na}$ forma integral, a relação acima fica:

$$
\frac{d}{d t} \int_{V}(\vec{r} \times \vec{g}) d v+\oint_{S}(\vec{T} x \vec{r}) \cdot \vec{n} d a=0
$$

onde $\int_{V}(\vec{r} \times \vec{g}) d v$ é o momento angular do campo eletromagnético no volume $V$.

A relação acima expressa a conservação do momento angular do campo eletromagnético [Fr-05].

Quando se considera que o volume $V$ engloba todo o espaço e os campos caem de forma suficientemente rápida, a integral de superfície tende a zero e as cargas de Noether (3.38) são dadas por $L^{\beta \rho}=\int d^{3} x M^{4 \beta \rho}$, para $(\beta, \rho)=\{(2,1),(3,1),(3,2)\}$. As componentes $L^{\beta \rho}$ são dadas por:

$$
\begin{aligned}
& L^{21}=-L^{12}=\int d^{3} x M^{421}=\int d^{3} x\left(x^{2} \Theta^{41}-x^{1} \Theta^{42}\right)=\int d^{3} x\left(x^{2} g^{1}-x^{1} g^{2}\right), \\
& L^{31}=-L^{13}=\int d^{3} x M^{431}=\int d^{3} x\left(x^{3} \Theta^{41}-x^{1} \Theta^{43}\right)=\int d^{3} x\left(x^{3} g^{1}-x^{1} g^{3}\right)
\end{aligned}
$$

$\mathrm{e}$

$$
L^{32}=-L^{23}=\int d^{3} x M^{432}=\int d^{3} x\left(x^{3} \Theta^{42}-x^{2} \Theta^{43}\right)=\int d^{3} x\left(x^{3} g^{2}-x^{2} g^{3}\right),
$$

e correspondem às componentes do momento angular total $\vec{L}$ do campo eletromagnético. 
As cargas de Noether correspondentes às componentes temporais do tensor $L^{\beta \rho}$, para as quais $(\beta, \rho)=\{(4,1),(4,2),(4,3)\}$, são dadas por:

$$
L^{4 \rho}=\int d^{3} x M^{44 \rho}=\int d^{3} x\left(x^{4} \Theta^{4 \rho}-x^{\rho} \Theta^{44}\right)=\int d^{3} x\left(x^{4} g^{\rho}\right)-\int d^{3} x\left(x^{\rho} u\right) .
$$

Assim, tem-se que:

$$
L^{4 \rho}=t P^{\rho}-\int d^{3} x\left(x^{\rho} u\right)
$$

onde a integral $\int d^{3} x g^{\rho}=P^{\rho}$ é a componente $\rho=1,2,3$ do momento linear total do campo eletromagnético.

Para se interpretar fisicamente essa quantidade conservada, considera-se o movimento de um sistema relativístico de partículas [Go-80] e tenta-se verificar as semelhanças com o resultado acima.

$\mathrm{Na}$ mecânica clássica, sabe-se que o momento angular total é conservado em um sistema isolado de partículas. Nessa demonstração, admite-se a validade da terceira lei de Newton. Tem-se, ainda, que o momento angular é independente do ponto de referência quando se adota o referencial com origem no centro de massa do sistema [Th-04].

Para o caso de uma partícula relativística, pode-se definir, no espaço-tempo de Minkowski, o tensor de segunda ordem e anti-simétrico $w^{\mu \nu}$, dado por:

$$
w^{\mu v}=x^{\mu} p^{v}-x^{v} p^{\mu}
$$

onde $p^{\mu}$ corresponde às componentes do quadrivetor momento-energia da partícula. Dessa definição, tem-se que o tensor $w^{i j}(i, j=1,2,3)$ é identificado com as componentes do momento angular da partícula.

Para se manter a covariância, a equação de movimento para $w^{\mu \nu}$ é dada em termos do tempo próprio $\tau$ da partícula na forma: 


$$
\frac{d w^{\mu v}}{d \tau}=\left(v^{\mu} p^{v}-v^{v} p^{\mu}\right)+\left(x^{\mu} K^{v}-x^{v} K^{\mu}\right),
$$

onde $K^{\alpha}=\frac{d p^{\alpha}}{d \tau}$ é a componente $\alpha$ da força de Minkowski.

Como as componentes do quadrivetor momento-energia são $p^{\alpha}=m v^{\alpha}$, onde $v^{\alpha}$ é a componente $\alpha$ da quadrivelocidade da partícula, tem-se que o primeiro termo do lado direito de (6.6) se anula. Assim, tem-se que:

$$
\frac{d w^{\mu \nu}}{d \tau}=\left(x^{\mu} K^{v}-x^{v} K^{\mu}\right)=N^{\mu v},
$$

onde $N^{\mu \nu}$ pode ser entendido como uma generalização relativística do torque, já que a expressão acima se reduz ao torque no limite não-relativístico.

Para um sistema de $n$ partículas, define-se o quadritensor de momento angular total do sistema, $W^{\mu \nu}$, dado por:

$$
W^{\mu \nu}=\sum_{n} w_{n}^{\mu \nu}
$$

Neste caso, uma equação de movimento para $W^{\mu \nu}$ não é simples de ser encontrada, uma vez que cada partícula possui seu tempo próprio. Para um sistema isolado em que as partículas não interagem umas com as outras ou com campos externos, tem-se que $w^{\mu \nu}$ é conservado para cada partícula, de acordo com a relação (6.7), o que implica na conservação de $W^{\mu v}$. Também, no caso de uma interação por colisões binárias, isto é, se a interação se dá somente entre pares de partículas, pode-se ter a conservação de $W^{\mu v}$. Isso ocorre em decorrência da questão relativística da simultaneidade, pois, no momento da interação, considera-se que as partículas se movem conjuntamente e, portanto, possuem o mesmo tempo próprio. Nessa situação, pode-se utilizar a equação (6.7) para a soma dos seus momentos angulares. No choque, como as forças impulsivas de contato são iguais e opostas, tem-se que 
a soma dos torques impulsivos se anula. Deste modo, tem-se que o momento angular relativístico também é conservado neste tipo de colisão.

Tomando-se as componentes temporais do tensor $W^{\mu v}$, ou seja, $W^{4 i}=-W^{i 4}$ $(i=1,2,3)$, e substituindo-se a definição (6.5) em (6.8), tem-se que:

$$
W^{4 i}=\sum_{n} w_{n}^{4 i}=\sum_{n}\left(x_{n}^{4} p_{n}^{i}-x_{n}^{i} p_{n}^{4}\right)
$$

Num dado sistema de referência $S$, tem-se: $x_{n}^{4}=t(c=1)$. A componente temporal do quadrivetor energia-momento é dada por $p_{n}^{4}=E_{n}$. Portanto, $W^{4 i}$ pode ser escrita na forma:

$$
W^{4 i}=\sum_{n}\left(t p_{n}^{i}-x_{n}^{i} E_{n}\right)=t P^{i}-\sum_{n} x_{n}^{i} E_{n}
$$

onde $P^{i}=\sum_{n} p_{n}^{i}$ é a i-ésima componente do momento linear total do sistema de partículas.

O conceito de centro de massa de um sistema de $n$ partículas desempenha um papel importante em mecânica clássica. Na mecânica relativística, devido à dependência da massa e do espaço com a velocidade, o centro de massa depende do referencial escolhido. Nessa situação, utiliza-se o chamado referencial do centro do momento $S_{P}$. Neste sistema de referência, a parte espacial do quadrivetor momento se anula e nele muitos problemas ficam mais fáceis de serem resolvidos [Ri-91].

Em $S_{P}$, tem-se que $P^{i}=0$ e a equação (6.9) fica:

$$
W^{4 i}=-\sum_{n} x_{n}^{i} E_{n}
$$

Para um sistema de $n$ partículas em que o momento angular relativístico total $W^{\mu \nu}$ se conserva, tem-se que $W^{4 i}=$ constante, de modo que, pela relação acima, $\sum_{n} x_{n}^{i} E_{n}$ é 
constante. Como o momento linear total também é conservado, tem-se que a energia total, $E=\sum_{n} E_{n}$, também é conservada. Assim, pode-se definir um ponto $\chi^{i}$, denominado de centro de energia do sistema [Bo-05, Bo-09], dado pela relação:

$$
\chi^{i}=\frac{\sum_{n} x_{n}^{i} E_{n}}{\sum_{n} E_{n}} .
$$

No limite não relativístico, e para $c=1$, a energia total de cada partícula está relacionada com sua massa na forma $E_{n}=m_{n}$, de modo que a relação acima fica reduzida ao centro de massa do sistema:

$$
\chi^{i}=\frac{\sum_{n} x_{n}^{i} m_{n}}{\sum_{n} m_{n}}=\frac{\sum_{n} x_{n}^{i} m_{n}}{M}
$$

onde $\sum_{n} m_{n}=M$ é a massa total do sistema de partículas. Deste modo, tem-se que o centro de energia do sistema de $n$ partículas se reduz, na situação clássica, ao centro de massa.

Pode-se, agora, retornar à expressão (6.4) para a componente temporal do momento angular total do campo eletromagnético, $L^{4 \rho}$, e verificar que ela é semelhante à relação (6.9), para a componente temporal do momento angular de um sistema de partículas, $W^{4 i}$. Com isso em mente, pode-se dividir (6.4) pela energia total do campo eletromagnético, $U=\int d^{3} x u$, obtendo-se a expressão:

$$
\frac{t P^{\rho}}{\int d^{3} x u}-\frac{\int d^{3} x\left(x^{\rho} u\right)}{\int d^{3} x u}=\text { constante }
$$

O segundo termo da relação acima é igual às três componentes $(\rho=1,2,3)$ da posição do centro de energia do campo: 


$$
\chi^{\rho}=\frac{\int d^{3} x\left(x^{\rho} u\right)}{\int d^{3} x u}
$$

Substituindo-se $\chi^{\rho}$ na relação (6.10), e considerando-se a energia total $U=\int d^{3} x u$ do campo, tem-se que:

$$
\chi^{\rho}=\frac{t P^{\rho}}{U}+\text { constante } \quad \text { ou } \quad \frac{d \chi^{\rho}}{d t}=\frac{P^{\rho}}{U} .
$$

Como o momento linear total $P^{\rho}$ e a energia total $U$ do campo eletromagnético são constantes, a relação acima implica que o centro de energia do campo eletromagnético permanece em movimento uniforme, com velocidade $P^{\rho} / U$.

Esse paralelo que se apresentou entre a conservação do momento angular do campo eletromagético e a de um sistema de partículas indica que a equação de continuidade (6.3) para $(\beta, \rho)=\{(4,1),(4,2),(4,3)\}$ expressa a constância da velocidade do centro de energia do campo eletromagnético.

Conclui-se, então, que as leis de conservação do momento angular e do centro de energia para o campo eletromagnético decorrem das simetrias por rotações no espaço-tempo.

\subsection{Lei de conservação associada às dilatações no espaço-tempo.}

A lei de conservação para o campo eletromagnético associada às dilatações é determinada a partir das transformações infinitesimais (4.17):

$$
x^{\prime \mu}=(1+\lambda) x^{\mu}, \mu=1,2,3,4
$$

definidas em termos do parâmetro $\varepsilon^{11}=\lambda<<1$ e das quatro funções (4.25): $\xi_{11}^{\mu}(x)=x^{\mu}$, que, substituídas na equação de continuidade (3.37), resultam na expressão: 


$$
\frac{\partial\left[\Theta^{\mu}{ }^{x^{\sigma}}\right]}{\partial x^{\mu}}=0 \quad(\mu, \sigma=1,2,3,4)
$$

ou seja:

$$
x^{\sigma} \frac{\partial \Theta^{\mu}}{\partial x^{\mu}}+\Theta_{\mu}^{\mu}=0
$$

O primeiro termo dessa expressão se anula devido a (6.1) para as translações. O segundo termo também se anula, pois o tensor de energia-momento possui traço nulo, como mostra (6.2). A lei de conservação associada à simetria de escala (ou de dilatação) do campo eletromagnético está ligada ao traço de $\Theta_{\sigma}^{\mu}{ }^{[\mathrm{Pl}-74]}$.

De (6.11), tem-se que a corrente de Noether para a dilatação é dada por $D^{\mu}(x)=\Theta^{\mu} \sigma^{x^{\sigma}}$, de modo a se obter a seguinte carga conservada (3.38):

$$
D=\int d^{3} x D^{4}(x)=\int d^{3} x \Theta^{4} x^{\sigma}=\int d^{3} x(\vec{r} \cdot \vec{g}-u t) .
$$

Procedendo-se, agora, como no item anterior, tenta-se obter uma interpretação física para a constante de movimento acima, examinando-se a simetria por dilatação na mecânica. Um exemplo simples envolve a determinação da corrente de dilatação para o movimento de uma partícula livre relativística [Ka-62] (no apêndice B, encontra-se uma apresentação resumida sobre a simetria de dilatação na mecânica clássica). Para esta partícula, a Lagrangiana é dada, em unidades naturais, pela relação (3.17):

$$
L=-m \sqrt{1-v^{2}}=-m \gamma^{-1},
$$

de onde se tem que:

$$
\frac{\partial L}{\partial v^{i}}=\frac{m v^{i}}{\sqrt{1-v^{2}}}=p^{i} .
$$


Para a dilatação, a variação infinitesimal da coordenada $x^{\mu}$ é dada por $\delta x^{\mu}=\lambda x^{\mu}$. Assim, substituindo-se os momentos (6.12) e $\delta x^{\mu}$ na relação (3.30) para a carga de Noether para sistemas discretos, obtém-se:

$$
\frac{d}{d t}[(\vec{p} \cdot \vec{v}-L) t-\vec{r} \cdot \vec{p}]=\frac{d}{d t}(E t-\vec{r} \cdot \vec{p})=0
$$

onde $\vec{p}=\left(p^{1}, p^{2}, p^{3}\right)$ é o momento linear e $E=\vec{p} \cdot \vec{v}-L=\left(p^{2}+m^{2}\right)^{1 / 2}$ é a energia total da partícula livre.

Deste modo, tem-se que a quantidade conservada é dada por:

$$
D=x^{\mu} p_{\mu}=\vec{r} \cdot \vec{p}-E t
$$

O movimento da partícula livre pode ser descrito pela relação $(c=1)$ :

$$
\vec{r}(t)=\frac{\vec{p}}{E} t+\vec{r}_{O}
$$

onde o vetor $\vec{r}_{O}$ é o vetor posição inicial da partícula. A substituição dessa relação em (6.13) leva a:

$$
D=\vec{r}_{0} \cdot \vec{p}-\frac{m^{2}}{E} t
$$

Esse resultado indica que $D$ é uma constante de movimento para a partícula livre somente para os limites em que $m \rightarrow 0$ ou $E \rightarrow \infty$. Assim a simetria de escala somente é verificada para partículas não massivas ou para altas energias [Ka-08].

É interessante a comparação desse resultado com uma situação em que a massa de repouso da partícula é nula como é o caso do fóton, a partícula mediadora da interação eletromagnética [Ri-02]. Para examinar esse caso de forma mais clara, explicitam-se aqui as constantes $h$ (constante de Planck) e $c$ (velocidade da luz). Para um fóton de frequência $v$, a 
energia é dada por $E=h v$ e o momento por $p=h v / c$. Nessa situação, para o fóton, o quadrimomento é dado por [Ri-91, Le-07]:

$$
P=\left(\vec{p}, \frac{E}{c}\right)=\frac{h v}{c}(\hat{n}, 1)
$$

onde $\hat{n}$ é o versor ao longo da direção de propagação. Nesse caso, $P$ é um quadrivetor tipo luz, ou seja, $P^{2}=0$. Considerando-se a frequência angular $\omega=2 \pi v$ e o vetor de onda $\vec{k}=\frac{\omega}{c} \hat{n}$, a relação acima fica na forma:

$$
P=\hbar\left(\vec{k}, \frac{\omega}{c}\right),
$$

onde $\hbar=h / 2 \pi$. Tem-se, então, que a constante de movimento $D$ definida pelo teorema de Noether para o fóton [Ri-02] é dada por:

$$
D=x^{\mu} p_{\mu}=\hbar(\vec{r} \cdot \vec{k}-\omega t)
$$

Para o campo eletromagnético, onde $E=p c,(6.13)$ é dada por:

$$
D=r p-E t=p(r-c t),
$$

e para o fóton onde $E=\hbar \omega, p=\hbar k$ e $\omega=c k$, a mesma relação toma a forma:

$$
D=r p-E t=\hbar k(r-c t) .
$$

Se, em $t=0$, uma frente de onda, ou um fóton, parte da posição $r_{0}$, tem-se que: $r=r_{0}+c t$. Deste modo, as duas últimas relações ficam:

$$
D=p(r-c t)=p r_{0} \quad \text { ou } \quad D=\hbar k(r-c t)=\hbar k r_{0},
$$


relações estas que estão de acordo com a relação (6.15) para $m \rightarrow 0$.

Finalizando, verificou-se que a interação eletromagnética possui uma lei de conservação associada à simetria de dilatação no espaço-tempo e isso está diretamente ligado ao fato da partícula mediadora dessa interação, o fóton, ter massa de repouso nula. É interessante notar que campos massivos já não apresentam simetria de escala como é o caso do campo de Procca [Gr-96, Ja-99], que representa uma extensão da teoria de Maxwell cujas partículas mediadoras têm massa não nula.

\subsection{Leis de conservação associadas às transformações conformes especiais no espaço- tempo.}

Para se determinar as leis de conservação para o campo eletromagnético associadas às transformações conformes especiais, considera-se a relação (4.18):

$$
x^{\prime \mu}=x^{\mu}+\left(2 x^{\beta} x^{\mu}-x_{v} x^{v} \eta^{\mu \beta}\right) s_{\beta}, \operatorname{com} \beta, \mu=1,2,3,4
$$

$\mathrm{Na}$ transformação acima, tem-se os parâmetros infinitesimais $\varepsilon^{r}<<1, r=12, \ldots, 15$, $\operatorname{com} \varepsilon^{12}=s_{1}, \varepsilon^{13}=s_{2}, \varepsilon^{14}=s_{3}, \varepsilon^{15}=s_{4}$, e as funções $\xi_{r}^{\mu}(x)$, dadas por (4.26):

$$
\xi_{r}^{\mu}(x)=2 x^{\beta} x^{\mu}-x_{v} x^{v} \eta^{\mu \beta}
$$

Assim, para a transformação conforme especial, a equação da continuidade (3.37) fica:

$$
\frac{\partial\left(2 x^{\beta} x^{\sigma} \Theta_{\sigma}^{\mu}-x_{v} x^{\nu} \Theta^{\mu \beta}\right)}{\partial x^{\mu}}=0 .
$$

As correntes para esta simetria são dadas por $I^{\mu \beta}(x)=2 x^{\beta} x^{\sigma} \Theta^{\mu}{ }_{\sigma}-x_{v} x^{v} \Theta^{\mu \beta}$ e as quantidades conservadas (3.38) ficam na forma: 


$$
I^{\beta}=\int d^{3} x I^{4 \beta}=\int d^{3} x\left[2 x^{\beta}(\vec{g} \cdot \vec{r}-u t)-\left(\vec{r}^{2}-t^{2}\right) g^{\beta}\right] .
$$

Desenvolvendo-se (6.16), verifica-se que:

$$
2 x^{\beta} \partial_{\mu}\left[\left(x^{\sigma} \Theta^{\mu}\right)\right]-x_{\nu} x^{\nu} \partial_{\mu} \Theta^{\mu \beta}+2 x_{\mu}\left(\Theta^{\beta \mu}-\Theta^{\mu \beta}\right)=0
$$

Os três termos da relação acima correspondem a termos da equação de continuidade já vistos para dilatações, translações e rotações, respectivamente, ou seja: o primeiro termo se anula devido a (6.11) para dilatações, o segundo, devido a (6.1) para translações e o terceiro termo, devido à simetria do tensor $\Theta^{\mu \beta}$ ligado às rotações.

Deste modo, verifica-se que a equação de continuidade para as transformações conformes especiais é uma consequência das outras três equações e não representa nenhuma nova lei de conservação para o campo eletromagnético.

Como o primeiro termo da relação acima está diretamente relacionado à equação de continuidade para dilatações (6.11), tem-se que a simetria por transformações conformes especiais também é válida somente para campos não massivos, como é o caso do campo eletromagnético. No apêndice $\mathrm{C}$, como foi feito para as dilatações, examina-se a simetria por transformações conformes especiais na mecânica clássica e relativística, onde se verifica que, para estas transformações, há quantidades que são conservadas para partículas com massa de repouso nula ou para altas energias.

Finalizando, verifica-se que, das quinze expressões de divergência nula para o campo eletromagnético, onze expressões realmente representam leis de conservação e as quatro restantes se reduzem às anteriores. Em síntese:

a) A equação (6.1):

$$
\partial_{\mu} \Theta^{\mu \sigma}=0, \operatorname{com} \sigma=1,2,3,4
$$

para as translações no espaço-tempo, está associada à conservação da energia e do momento linear. 
b) A equação (6.3):

$\partial_{\mu}\left(x^{\beta} \Theta^{\mu \rho}-x^{\rho} \Theta^{\mu \beta}\right)=0, \operatorname{com}(\beta, \rho) \in\{(2,1),(3,1),(3,2),(4,1),(4,2),(4,3)\}$,

para as rotações, está associada à conservação do momento angular e ao movimento uniforme do centro de energia do campo eletromagnético.

c) A equação (6.11):

$$
\partial_{\mu}\left(\Theta^{\mu} x^{\sigma}\right)=0, \sigma=1,2,3,4
$$

é a lei de conservação para o campo eletromagnético associada às transformações por dilatações no espaço-tempo.

d) A equação (6.16):

$$
\partial_{\mu}\left(2 x^{\beta} x^{\sigma} \Theta_{\sigma}^{\mu}-x_{v} x^{v} \Theta^{\mu \beta}\right)=0, \operatorname{com} \beta=1,2,3,4
$$

para as transformações conformes especiais, é uma consequência das leis anteriores de conservação para o campo eletromagnético. 


\section{CAPÍTULO VII}

\section{RESULTADOS E CONCLUSÕES}

Apresenta-se, a seguir, um resumo dos principais resultados e conclusões deste trabalho, onde se fez um estudo sobre a invariância conforme do campo eletromagnético clássico e as leis de conservação a ele associadas.

No capítulo II foi apresentada, inicialmente, uma introdução às transformações conformes. Primeiramente, discutiram-se transformações em duas dimensões, entre elas as associadas ao plano complexo, onde se mostraram propriedades destas transformações, úteis na resolução de problemas bidimensionais em Física. A seguir, passou-se à generalização de relações válidas em duas dimensões para espaços métricos de $n$ dimensões e apresentou-se a projeção estereográfica, que mapeia pontos de uma superfície esférica para um plano e é importante em cartografia. Abordaram-se, finalmente, espaços de quatro dimensões, importantes para o estudo da invariância conforme das equações de Maxwell.

Também no capítulo II, apresentou-se uma síntese histórica, onde se discutiu como as transformações conformes foram desenvolvidas e aplicadas em diversas épocas e áreas do conhecimento, desde os tempos gregos até o início da década de 1970, quando a invariância conforme passou a ser utilizada correntemente em teorias quânticas de campos. Este apanhado histórico mostrou como diversas circunstâncias levaram às descobertas, desenvolvimentos e aplicações das transformações conformes em geometria, cartografia e Física. Verifica-se também uma visível ligação entre o aumento do comércio, as grandes navegações, a cartografia e o estudo das transformações conformes. Neste contexto, podem-se citar: o mapeamento da esfera celeste e a construção do astrolábio pelos gregos; o desenvolvimento da cartografia durante e após o Renascimento europeu; os estudos sobre geometria diferencial e funções complexas durante os séculos XVIII e XIX. Essa ligação entre questões econômicas, cartografia e mapeamento gerou o desenvolvimento de áreas como a instrumentação, astronomia e matemática, onde se tem a participação de nomes como Euler, Lagrange, Gauss, Riemann, Liuville, Lie e muitos outros.

No capítulo III, revisou-se a formulação covariante da eletrodinâmica, o formalismo Lagrangiano e o teorema de Noether para campos, instrumentos teóricos utilizados nos capítulos seguintes para a verificação da invariância conforme na eletrodinâmica e das leis de conservação associadas ao campo eletromagnético. Apresentou-se o formalismo Lagrangiano 
aplicado a Eletrodinâmica. Seguindo-se a apresentação do teorema de Noether para campos, foi feita a aplicação deste para o campo eletromagnético sem fontes, onde se obteve a equação de continuidade e foram explicitadas as correntes e cargas conservadas. O teorema de Noether é o instrumento central utilizado no capítulo VI.

No capítulo IV, abordou-se a determinação das transformações conformes em espaços planos de $n$ dimensões. Chegou-se a equações diferenciais que permitem a determinação de funções que caracterizam as transformações conformes infinitesimais para espaços planos. Como aplicação simples e esclarecedora, bem como para teste das equações obtidas, as equações foram aplicadas para duas dimensões, situação em que se tem um número infinito de transformações e chega-se às conhecidas relações de Cauchy-Riemann. Também com base nas equações mostradas no primeiro item do capítulo, mostrou-se, que, para dimensões maiores ou iguais a três $(n \geq 3)$, as transformações conformes ficam restritas ao grupo das translações, rotações, dilatações e transformações conformes especiais, bem como composições destas transformações. No espaço-tempo de Minkowski ( $n=4)$, mostrouse que as transformações conformes infinitesimais possuem quinze parâmetros. Por fim, fezse uma discussão do ponto de vista físico das transformações de Lorentz-Poincaré (que relacionam dois referenciais inerciais) e formam um subgrupo das transformações conformes de coordenadas obtidas. Esse subgrupo relaciona referenciais inerciais e sua linearidade está ligada à homogeneidade e isotropia do espaço-tempo. Discutiram-se, também, as transformações de Lorentz na forma que são apresentadas em textos clássicos da teoria da relatividade.

No capítulo $\mathrm{V}$ foi abordada a invariância conforme das equações da eletrodinâmica. Partiu-se de uma discussão sobre os sistemas de coordenadas curvilíneas no espaço-tempo; e, partindo-se da equação de movimento de uma partícula em um sistema cartesiano, chegou-se à mesma equação em um sistema curvilíneo. Chegou-se naturalmente à ideia de derivada covariante, com a qual as equações de Maxwell foram escritas em uma forma que mantivesse sua covariância no grupo mais geral das coordenadas curvilíneas. Com essas equações escritas nessa forma mais geral, chegou-se à condição a ser imposta para que as equações de Maxwell mantivessem a mesma forma que as que aparecem em coordenadas cartesianas. Como conclusão, verificou-se que a condição obtida é a mesma que gera as transformações conformes obtidas no Capítulo II.

No último capítulo, fez-se uma discussão sobre as leis de conservação associadas à invariância conforme das equações de Maxwell, a partir da equação de continuidade obtida via teorema de Noether e das equações que caracterizam as transformações conformes 
infinitesimais. Para o teorema de Noether, o núcleo é o tensor de energia momento $\Theta^{\mu \sigma}$ e as transformações envolvem 15 parâmetros $(r=1, \ldots, 15)$ e as funções $\xi_{r}^{\mu}(x)(\mu=1, \ldots, 4)$.

Primeiramente, consideraram-se as simetrias por translações no espaço-tempo. Verificou-se que elas estão associadas às leis de conservação do momento linear $(r=1,2,3)$ e da energia $(r=4)$ do campo eletromagnético.

Verificou-se, em seguida, que as simetrias devidas às rotações no espaço-tempo implicam nas leis de conservação do momento angular $(r=5,6,7)$ para o campo. Para $(r=8,9,10)$, a interpretação física das grandezas conservadas envolveu a observação do movimento relativístico de um sistema de partículas onde se procurou buscar semelhanças entre as relações obtidas para o campo eletromagnético e as do sistema mecânico. Dessa comparação, observou-se que as três últimas grandezas conservadas estavam associadas à velocidade do centro de energia do campo.

Para as dilatações no espaço-tempo $(r=11)$, verificou-se que a quantidade conservada para o campo eletromagnético não apresentou um significado físico que pudesse ser compreendido de modo direto. Como tentativa de aclarar esse significado, examinou-se a simetria de dilatação para o movimento de uma partícula relativística livre. Nessa situação simples, chegou-se a uma quantidade que somente seria conservada para os limites de massa nula, $m \rightarrow 0$, ou altas emergias, $E \rightarrow \infty$. Assim, verificou-se que a simetria de escala somente é observada para partículas não massivas ou para altas energias. Usando na quantidade obtida para a partícula livre as relações quânticas $E=\hbar \omega$ e $p=\hbar k$ para fótons, chega-se a uma constante. O mesmo ocorre quando se utiliza a relação energia-momento para o campo $E=p c$. Esse resultado indica que campos não massivos apresentam simetria de escala. Verificou-se que, para fótons, a quantidade conservada está diretamente relacionada à fase da onda associada, que é um invariante de Lorentz. Assim, evidenciou-se a existência da simetria de escala para o campo eletromagnético, pois este campo tem partículas mediadoras com massa nula.

Para as transformações conformes especiais, verificou-se que as leis de conservação resultantes são consequências das leis anteriores de conservação para o campo eletromagnético. No entanto, aplicando-se a transformação conforme especial para uma partícula livre relativística pode-se verificar que estas transformações possuem a mesma propriedade das transformações de escala, ou seja, têm-se quantidades conservadas somente para o caso da massa da partícula ser nula ou para altas energias. Há, portanto, uma conclusão 
análoga ao caso da simetria de escala, ou seja, a simetria por transformação conforme especial somente existe para campos não-massivos como o eletromagnético ou para partículas de altas energias.

Assim, conclui-se que, diferentemente das simetrias por translações ou rotações no espaço-tempo, as simetrias de dilatação e por transformações conformes especiais são válidas para campos não massivos ou, no caso de partículas, podem ser vistas como simetrias aproximadas da natureza, válidas para altas energias. 


\section{APÊNDICE A}

\section{AS TRANSFORMAÇÕES CONFORMES ESPECIAIS E O MOVIMENTO UNIFORMEMENTE ACELERADO.}

Como se apresentou no Capítulo II, historicamente, após a verificação de que as equações da eletrodinâmica são invariantes por translações, rotações, dilatações e pelas transformações conformes especiais, passou-se à interpretação física dessas simetrias. As transformações conformes especiais, menos conhecidas, passaram a ser foco dessas interpretações. No trabalho de Bateman [Ba-10] há uma menção sobre transformações de coordenadas entre referenciais com aceleração constante, e no artigo de Cunningham [Cu-10] há uma tentativa de extensão do princípio da relatividade restrita para referenciais com aceleração constante baseada na invariância das equações do eletromagnetismo pela transformação de inversão. A partir da terceira década do século passado até o início da década de 1960, vários trabalhos foram publicados tentando interpretar a transformação conforme especial como uma transformação entre um referencial inercial e outro com aceleração constante [En-36, Gu-61, Hi-45, Hi-47, In-52, Jo-61, Pa-36]. Segue-se uma pequena discussão sobre essa questão.

O movimento relativo entre dois referenciais inerciais no espaço-tempo plano é tratado pela teoria da relatividade restrita. Como visto no capítulo IV, seção 4.5, as transformações de Lorentz-Poincaré fornecem a relação entre as coordenadas de dois eventos medidos nesses referenciais. O movimento de um referencial com aceleração constante $\mathcal{A}_{0}$ também pode ser tratado do ponto de vista da relatividade restrita [Ca-06, De-87, Se-06].

Considerando-se as transformações de Lorentz (4.28) entre dois referencias inerciais $S$ e $S^{\prime}$ que se movem com velocidade relativa constante $v$, tem-se que a relação de transformação para a aceleração de uma partícula que se move com velocidade $u_{x}=\frac{d x}{d t} \mathrm{e}$ aceleração $A_{x}=\frac{d u_{x}}{d t}$ é dada por:

$$
\mathcal{A}^{\prime}{ }_{x}=\frac{d u^{\prime}{ }_{x}}{d t^{\prime}}=\frac{\left(1-v^{2}\right)^{3 / 2}}{\left(1-v u_{x}\right)^{3}} \mathcal{A}_{x},
$$


onde se utilizou no cálculo acima a relação para transformação de velocidades: $u_{x}^{\prime}=\frac{u_{x}-v}{1-u_{x} v}$.

Para uma partícula que possui uma aceleração constante $\mathcal{A}^{\prime}{ }_{x}=\mathcal{A}_{0}$ em relação a um referencial inercial que instantaneamente a acompanha, ou seja, em que $u_{x}=v$, tem-se que:

$$
\mathcal{A}_{x}=\mathcal{A}_{0}\left(1-u_{x}^{2}\right)^{3 / 2}
$$

Considerando-se que em $t=0$ a partícula está em repouso na origem, a integração da relação acima implica que:

$$
\begin{gathered}
\int_{0}^{t} \mathcal{A}_{0} d t=\int_{0}^{t} \frac{d}{d t}\left(\frac{u_{x}}{\left(1-u_{x}^{2}\right)^{1 / 2}}\right) d t=> \\
u_{x}=\frac{\mathcal{A}_{0} t}{\sqrt{1+\left(\mathcal{A}_{0} t\right)^{2}}} .
\end{gathered}
$$

Integrando-se novamente, tem-se que:

$$
x(t)=\frac{1}{\mathcal{A}_{0}}\left(\sqrt{1+\left(\mathcal{A}_{0} t\right)^{2}}-1\right) .
$$

Rearranjando-se a expressão acima, obtém-se:

$$
\left(\mathcal{A}_{0} x+1\right)^{2}-\left(\mathcal{A}_{0} t\right)^{2}=1
$$

A equação acima tem a forma de um ramo de hipérbole no diagrama de espaçotempo $(x, t)$ e, por este motivo, o movimento uniformemente acelerado de uma partícula relativística é chamado de movimento hiperbólico [Di-98, Ri-06]. Este tipo de movimento está associado à ação de uma força constante sobre a partícula, e ocorre, por exemplo, quando elétrons são acelerados a velocidades relativísticas por meio de um campo elétrico constante e uniforme [Go-80]. O estudo do movimento uniformemente acelerado via relatividade restrita 
pode ser considerado como uma primeira aproximação da teoria da relatividade geral, tendo em vista o princípio de equivalência [Ei-07], o qual estabelece que em uma região suficientemente pequena do espaço e para uma duração também pequena de tempo um campo gravitacional uniforme é equivalente a um referencial com aceleração constante.

Pode-se considerar, agora, a transformação conforme especial (4.22):

$$
x^{\prime} \mu=\frac{x^{\mu}-(x . x) s^{\mu}}{1-2(s . x)+(s . s)(x . x)},
$$

como a transformação entre as coordenadas relativas ao referencial em que a partícula está instantaneamente em repouso e o referencial do laboratório (inercial) [Ca-11, Co-97, Fu-62a]. Para a partícula (instantaneamente em repouso na origem), tem-se que $x^{\mu}=(0,0,0, t)$. Considerando-se os parâmetros de transformação, $s$, dados por $s^{\mu}=(s, 0,0,0)$, tem-se que as coordenadas $x^{\prime \mu}$ são dadas por:

$$
x^{\prime}=\frac{s t^{2}}{1-s^{2} t^{2}}, \quad y^{\prime}=0, \quad z^{\prime}=0 \quad \text { e } \quad t^{\prime}=\frac{t}{1-s^{2} t^{2}} .
$$

Escrevendo-se $x^{\prime}$ em função de $t^{\prime}$, tem-se:

$$
\left(2 s x^{\prime}+1\right)^{2}-\left(2 s t^{\prime}\right)^{2}=1
$$

Comparando-se a relação acima com (A.1), vê-se que esta é a equação do movimento hiperbólico nas coordenadas $\left(x^{\prime}, t^{\prime}\right)$ do laboratório com o parâmetro de transformação dado por $s=\frac{\mathcal{A}_{0}}{2}$.

O resultado acima ilustra a interpretação para as transformações conformes especiais como transformações que relacionam as coordenadas de referenciais inerciais e referenciais uniformemente acelerados ao longo de uma coordenada espacial. Essa interpretação foi adotada a partir da metade dos anos de 1930 até os anos de 1960, quando essas transformações passaram a ser interpretadas como transformações locais de escala no espaçotempo [Ka-08]. 


\section{APÊNDICE B}

\section{ALGUNS ASPECTOS DA TRANSFORMAÇÃO DE DILATAÇÃO NA MECÂNICA CLÁSSICA.}

Em mecânica clássica, a simetria de escala está associada à invariância das equações de movimento de um sistema pela dilatação das coordenadas do espaço e do tempo [An-71, Go-08 , La-66], ou seja:

$$
\vec{r}^{\prime}=\alpha \vec{r}
$$

e

$$
t^{\prime}=\eta t
$$

onde $\alpha$ e $\eta$ são constantes reais. Esta simetria é também chamada de similaridade mecânica.

Para a discussão que se segue, toma-se o potencial do tipo $U(r)=b r^{\beta}(r=|\vec{r}|$ e $b=$ constante). Neste caso, a transformação (B.1) implica que:

$$
U^{\prime}\left(r^{\prime}\right)=U^{\prime}(\alpha r)=\alpha^{\beta} U(r)
$$

Para uma partícula de massa $m$ sujeita a um potencial $U(r)$ tem-se que:

$$
m \frac{d^{2} r}{d t^{2}}=-\frac{d U(r)}{d r}
$$

Substituindo-se as relações (B.1), (B.2) e (B.3) na expressão acima, chega-se a $\eta=\alpha^{(1-\beta / 2)}$. Deste modo, tem-se que a relação (B2) fica na forma:

$$
t^{\prime}=\alpha^{(1-\beta / 2)} t
$$

Considerando-se a transformação acima e (B.1) para as coordenadas de espaço, temse que a velocidade da partícula toma a forma: 


$$
\dot{r}^{\prime}\left(t^{\prime}\right)=\frac{d r^{\prime}}{d t^{\prime}}=\alpha^{\beta / 2} \dot{r}(t)
$$

Assim, se a energia potencial do sistema é uma função homogênea de grau $\beta$ (B.3), tem-se como possibilidades de soluções trajetórias geometricamente similares, de acordo com as dilatações (B.1), (B.3), (B.4) e (B.5). Dessas observações, é interessante notar que é possível utilizar-se a similaridade mecânica para se obterem resultados conhecidos da mecânica clássica sem a necessidade de se utilizar as leis de Newton [La-66].

Examinam-se, agora, os efeitos da simetria de escala na Lagrangiana e na integral de ação. Para a partícula de massa $m$ sujeita ao potencial $U(r)=b r{ }^{\beta}$, a Lagrangiana é dada por:

$$
L=\frac{1}{2} m \dot{r}^{2}-b r^{\beta}
$$

Utilizando-se (B.1), (B.5) e considerando-se $\alpha$ na forma $\alpha=e^{\kappa}$ na Lagrangiana acima, tem-se que, para o sistema transformado, $L^{\prime}$ é dada por:

$$
L^{\prime}=e^{\kappa \beta} L
$$

Como a Lagrangiana (B.6) é multiplicada por uma constante, tem-se que as equações de movimento do sistema permanecem as mesmas.

$\mathrm{O}$ efeito da simetria de escala na ação pode ser verificado considerando-se a dilatação para o tempo (B.4) e a Lagrangiana transformada (B.7). Neste caso, tem-se que a ação fica invariante, ou seja, $\int L d t=\int L^{\prime} d t^{\prime}$, somente para $\beta=-2$.

Assim, tem-se que o único potencial na forma $U(r)=b{ }^{\beta}$ que exibe a simetria de escala no nível clássico ${ }^{1}$ é dado pelo grau de homogeneidade $\beta=-2$ [Go-05, Ka-68], ou seja, em que $L$ é dada por:

\footnotetext{
${ }^{1}$ Em mecânica clássica tem-se que interação delta de Dirac em duas dimensões $\delta^{2}(r)$ também exibe a simetria de escala.
} 


$$
L=\frac{1}{2} m \dot{r}^{2}-\frac{b}{r^{2}}
$$

Um sistema físico que possui este tipo de simetria é o de um campo de um dipolo elétrico, cujo potencial varia com $r^{-2}$ numa região afastada do centro do dipolo.

A quantidade conservada para esses sistemas pode ser obtida via o teorema de Noether, através da condição (3.30), isto é:

$$
\frac{d}{d t}\left[\left(\frac{\partial L}{\partial \dot{r}} \dot{r}-L\right) \delta t-\frac{\partial L}{\partial \dot{r}} \delta r\right]=0
$$

Para a Lagrangiana (B.8), tem-se que $\frac{\partial L}{\partial \dot{r}}=m \dot{r}=p$. As variações infinitesimais da coordenada $r$ e do tempo $t$ são dadas, respectivamente, por $\delta r=\kappa r$ e $\delta t=2 \kappa t$. Assim, obtém-se:

$$
\frac{d}{d t}\left(H t-\frac{p r}{2}\right)=0
$$

onde $H=p \dot{r}-L$ é a energia total da partícula. Deste modo, tem-se a seguinte quantidade conservada relativa à simetria de dilatação:

$$
D=H t-\frac{p r}{2}
$$




\section{APÊNDICE C}

\section{AS TRANSFORMAÇÕES CONFORMES ESPECIAIS NA MECÂNICA CLÁSSICA E RELATIVÍSTICA.}

Apresenta-se, a seguir, uma pequena discussão sobre as transformações conformes especiais na mecânica clássica e relativística. Nessa discussão, considera-se, inicialmente, o caso de uma partícula sujeita ao potencial $U(r)=\frac{b}{r^{2}}$, cuja Lagrangiana é dada por:

$$
L=\frac{1}{2} m \dot{r}^{2}-\frac{b}{r^{2}}
$$

Mostrou-se, no apêndice B, que, neste caso, a ação é invariante pelas transformações de dilatação $r^{\prime}=e^{\kappa} r$ e $t^{\prime}=e^{2 \kappa}$. Esta invariância implica na constante de movimento (B.9): $D=H t-\frac{1}{2} r p$.

Neste apêndice, consideram-se as seguintes transformações conformes para o tempo $t$ e para a coordenada espacial $r$ [Go-13],

$$
t^{\prime}=\frac{t}{1-\eta t}
$$

e

$$
r^{\prime}=\frac{r}{1-\eta t}
$$

onde $\eta$ é uma constante real.

A transformação conforme especial, (C.1), para $t$ ' pode ser entendida como uma inversão de $t$, seguida de uma translação $-\eta$ e de outra inversão:

$$
t \rightarrow \frac{1}{t} \rightarrow \frac{1}{t}-\eta \rightarrow \frac{1}{\frac{1}{t}-\eta}=\frac{t}{1-\eta t}
$$


As transformações infinitesimais associadas a (C.1) e (C.2) são dadas por:

$$
t^{\prime}=t+\eta t^{2}, \operatorname{com} \delta t=\eta t^{2}
$$

$\mathrm{e}$

$$
r^{\prime}=r+\eta r t, \text { com } \delta r=\eta r t
$$

Verifica-se, neste caso, que a integral da ação $S$ é quase-invariante [Le-07] sob estas transformações infinitesimais, ou seja:

$$
\delta S=\int_{t_{1}}^{t_{2}} L\left(r^{\prime}\left(t^{\prime}\right), \dot{r}^{\prime}\left(t^{\prime}\right), t^{\prime}\right) d t^{\prime}-\int_{t_{1}}^{t_{2}}\left[L(r(t), \dot{r}(t), t)+\eta \frac{d}{d t} g(r, t)\right] d t=0
$$

onde: $g(r, t)=\frac{m r^{2}}{2}$.

Sabe-se que as equações de movimento de um sistema não se alteram para Lagrangianas que diferem entre si pela derivada total em relação ao tempo de uma função genérica das coordenadas e do tempo. Considerando-se a variação da ação acima, tem-se que o teorema de Noether admite uma generalização que reflete esta propriedade das Lagrangianas equivalentes. Neste caso, a condição de Noether (3.30) fica na forma [Le-07]:

$$
\frac{d}{d t}\left\{\left(\frac{\partial L}{\partial \dot{r}} \dot{r}-L\right) \delta t-\frac{\partial L}{\partial \dot{r}} \delta r+\delta g\right\}=0
$$

Substituindo-se as variações $\delta t$ e $\delta r$ na relação acima, e considerando-se que $\frac{\partial L}{\partial \dot{r}}=m \dot{r}=p$ e $\delta g=\eta g$, obtém-se a seguinte quantidade conservada [Go-13, Ka-08]:

$$
I=2 H t^{2}-2 p r t+m r^{2}
$$

Esta constante de movimento, juntamente a constante (B.9) determinada no apêndice B para a dilatação, fornece a solução geral para $r(t)$. 
Estes aspectos e propriedades básicas das simetrias de escala e por transformações conformes especiais para potencial $U(r)=\frac{b}{r^{2}}$ formam as bases para a chamada mecânica conforme, desenvolvida a partir de trabalhos no início da década de 1970 [Ja-72, Al-76], e que ainda hoje continuam sendo foco de interesse [Go-13].

Considera-se, agora, o movimento de uma partícula livre relativística, cuja Lagrangiana é dada pela relação por (3.17).

Para as transformações conformes especiais, a partir de (4.18), tem-se que a variação infinitesimal da coordenada $x^{\mu}$ é dada por:

$$
\delta x=\left(2 x^{\beta}{ }_{x}^{\mu}-x_{v} x^{\nu} \eta^{\mu \beta}\right)_{\beta} .
$$

Substituindo-se os momentos (6.12) e as variações acima na relação (3.30), obtémse:

$$
\frac{d}{d t}\left[E\left(2{ }_{x} \beta_{t-x_{V}} x^{v} \eta^{4 \beta}\right)_{s \beta}-p_{i}\left(2{ }_{x} \beta_{x}{ }-x_{V} x^{v} \eta^{i \beta}\right)_{s \beta}\right]=0
$$

$\operatorname{com} i=1,2,3 ; \beta=1,2,3,4$ e $E=\vec{p} \cdot \vec{v}-L=\left(p^{2}+m^{2}\right)^{1 / 2}$.

Lembrando-se que $p^{4}=E$ e $x^{4}=t$, a relação acima pode ser rearranjada para se obter as quatro quantidades correntes conservadas (associadas a cada constante $s_{\beta}$ ) na forma compacta:

$$
{ }_{I}^{\beta}=x_{v^{x}}{ }^{\nu} p^{\beta}-2 x^{\beta} p_{v^{x}} x^{v}, \operatorname{com} \beta=1,2,3,4
$$

Considerando-se a corrente de dilatação $D$ (6.13) para a partícula livre, essas quatro correntes podem ser escritas nas formas:

$$
\vec{I}=\left(\vec{r}^{2}-t^{2}\right) \vec{p}-2 \vec{r} D \text { (componentes espaciais) }
$$

e

$$
I^{4}=\left(\vec{r}^{2}-t^{2}\right) E-2 t D \text { (componente temporal). }
$$


As relações acima mostram que, no caso da partícula livre relativística, a conservação das correntes associadas às transformações conformes especiais está diretamente ligada à conservação da corrente de dilatação.

Substituindo-se, agora, a relação (6.14) nas relações acima, tem-se que:

$$
\vec{I}=\vec{r}_{0}^{2} \cdot \vec{p}-2\left(\vec{r}_{0} \cdot \vec{p}\right) \vec{r}_{O}-\left(\frac{m^{2}}{E}\right)\left[\left(\frac{\vec{p}}{E}\right) t^{2}+2 \vec{r}_{0} \cdot t\right]
$$

e

$$
I^{4}=\vec{r}_{0}^{2} E+\left(\frac{m^{2}}{E}\right) t^{2} .
$$

Verifica-se que as relações acima são constantes de movimento somente para $m \rightarrow 0$ ou $E \rightarrow \infty$. Como no caso da transformação de dilatação no espaço-tempo, tem-se que a simetria associada às transformações conformes especiais também é válida somente para altas energias ou para partículas com massa de repouso nula, como no caso de fótons [Ri-02]. Neste último caso, as constantes (C.3) e (C.4) dependem da constante de dilatação $D=\hbar(\vec{r} \cdot \vec{k}-w t)$.

A análise acima para a partícula livre serviu para uma reinterpretação física das transformações conformes especiais a partir do início da década de 1960 [Ka-62, Ka-66, Ka-66a], substituindo a interpretação até então vigente, que as associava ao movimento uniformemente acelerado no espaço-tempo.

Considerando-se os elementos de linha associados às transformações conformes especiais e de escala no espaço-tempo, dadas, respectivamente, por:

$$
d s^{\prime 2}=v(x)^{-2} d s^{2}, \text { com } v(x)=1-2 s \cdot x+s^{2} x^{2}
$$

e

$$
d s^{2}=\lambda^{2} d s^{2}
$$

pode-se interpretar as transformações conformes especiais no espaço-tempo como transformações locais de escala [Ka-08, Ku-77], dependentes da posição, pois seu elemento de linha sofre uma dilatação proporcional a $v(x)^{-2}$. 


\section{REFERÊNCIAS}

[Ah-79] Ahlfors, L.V., Complex analysis: an introduction to the theory of analytic functions of one complex variable, $3^{\mathrm{a}}$ ed., McGraw-Hill, New York, 1979.

[Al-06] Albinus, M. and Ibragimov, N. H., On Conservation Laws of Electrodynamics, Archives of ALGA, ALGA Publications - Blekinge Institute of Technology Karlskrona, Sweden, 3, 33-48, 2006 (Tradução para o inglês do artigo de Bessel-Hagen, referência [Be-21]).

[Al-76] Alfaro, V. de, Fubini, S. and Furlan, G., Conformal Mechanics in Quantum Mechanics, Nuovo Cim., 34a, 569, 1976.

[An-71] Andersen, C. M. and Baeyer, Hans C. Von, Scaling and the Virial Theorem in Mechanics and Action-at-a-Distance Electrodynamics, Am. J. Phys., 39, 914, August, 1971.

[Ar-85] Arkfen, G. B., Mathematical Methods for Physicists, Academic Press, 1985.

[As-13] Disponível em: <http://astrolabeproject.com/downloads/Astrolabe_the_Missing_Ma nual.pdf>, acesso em 01.03.2013.

[Ba-09] Bateman, H., The Conformal Transformations of a Space of Four Dimensions and their Applications to Geometrical Optics, Proc. London Math. Soc. (Ser. 2) 7, 70, 1909.

[Ba-10] Bateman, H., The Transformation of the Electrodynamical Equations, Proc. London Math. Soc. 8, 223, 1910.

[Ba-74] Bakel'man, I. Y., Inversions, The University Chicago Press, 1974.

[Be-21] Bessel-Hagen, E., Über die Erhaltungssätze der Elektrodynamik, Mathem. Ann. 84, 258-276, 1921.

[Be-84] Beckers, J. and Sinzinkayo, S., Invariance under conformal and coordinate and point transformations, Physica 126A, 371-383, 1984.

[Be-91] Berggren, J.L., Ptolemy's Maps of Earth and the Heavens: A New Interpretation, Arch. Hist. Exact Sci. 43, 133-144, 1991.

[Bi-13] Disponível em: <http://objdigital.bn.br/acervo_digital/div_cartografia/cart 986513.jpg>, Biblioteca Digital do Patrimônio Ibero-Americano (BDPI), acesso em 01.03.2013.

[B1-00] Blair, D.E., Inversion Theory and Conformal Mapping, American Mathematical Society, 2000.

[B1-09] Blumenhagen, R. and Plauschinn, E., Introduction to Conformal Field Theory: with Applications to String Theory, Lect. Notes Phys. 779, Springer, Berlin Heidelberg, 2009. 
[Bo-05] Boyer, T. H., Illustrations of the relativistic conservation law for the center of energy, Am. J. Phys, 73 (10), October, 2005.

[Bo-09] Boyer, T. H., Illustrating some implications of the conservation laws in relativistic mechanics, Am. J. Phys, 77 (06), June, 2009.

[Br-13] Disponível em: 〈http://www.britannica.com/EBchecked/topic/22486/analysis\#>, acesso em 01.03.2013.

[Bu-88] Butkov, E., Física Matemática, Editora Guanabara Koogan S/A, 1988.

[Ca-06] Castillo, G. F. T., Uniformly Accelerated Observers in Special Relativity, Revista Mexicana de Física, 52(1), 2006.

[Ca-09] Camargo, V.L.V. de, Trajetórias sobre o globo terrestre: um estudo da geometria da esfera nos mapas cartográficos, Dissertação de mestrado, Unicamp, 2009.

[Ca-11] Calixto, M., Romero, E. P. and Aldaya, V., Coherent States of Accelerated Relativistic Quantum Particles, Vacuum Radiation and the Spontaneous Breakdown of the Conformal SU(2,2) Symmetry, J.Phys., A45, 2012.

[Cl-93] Clavius, C., Astrolabium, Romae, Impensis Bartholomaei Grassi. Ex Typographia Gambiana, 1593. Disponível em: Fondos Digitalizados de la Universidad de Sevilla $<$ http://fondosdigitales.us.es/books/>. Acesso em 01.03.2013.

[Co-67] Coxeter, H.S.M. e Greitzer, S.L., Geometry Revised, The Mathematical Association of America, 1967.

[Co-69] Coxeter, H. S. M., Introduction to Geometry, John Wiley \& Sons, 1969.

[Co-70] Coxeter, H. S. M., Inversive Geometry, Educational Studies in Mathematics, 3, 3-4, 1970.

[Co-97] Codirla, C. and Osborn, H., Conformal Invariance and Electrodynamics: Applications and General Formalism, Annals of Physics, 260(1), 91, 1997.

[Co-98] Coxeter, H. S. M., Non-Euclidean Geometry, The Mathematical Association of America, 1998.

[Cu-10] Cunningham, E., The Principle of Relativity in Electrodynamics and an Extension Thereof, Proc. London Math. Soc. 8, 77, 1910.

[De-87] Deslog, E. A. and Philpott, R. J., Uniformly Accelerated Reference Frames in Special Relativity, Am. J. Phys., 55(3), 1987.

[Di-75] Dirac, P. A. M., General Theory of Relativity, Wiley-Interscience Publication, 1975.

[Di-98] D’Inverno, R., Introducing Einstein's Relativity, Clarendon Press-Oxford, 1998. 
[Do-05] Doyle, K. M., Contributions of the Theory of Conformal Mapping to the Field of Electrical Engineering Education and Practice, Dissertation submitted in partial fulfillment of the requirements for the degree of Doctor of Philosophy, Columbia University, 2005.

[Ei-05] Einstein, A., Zur Elektrodynamik bewegter Körper, Annalen der Physik, 17, 891, 1905.

[Ei-07] Einstein, A., Uber das Relativtätsprinzip und die aus Demselben Gezogenen Folgerungen, Jahrb. Radioakt, 4, 411, 1907 - A tradução para o português pode ser encontrada na Rev. Bras. de Ens. de Fís., 27, 37, 2005, Sobre o Princípio da Relatividade e suas Implicações.

[Em-14] Emch, A., The Discovery of Inversion, Bulletin of the American Mathematical Society, 21, 1914.

[Em-15] Emch, A., Note on the Discovery of Inversion, Bull. Amer. Math. Soc., 21, 4, 1915.

[Em-29] Emch, A., Unpublished Steiner Manuscripts, The American Mathematical Monthly, $36,5,1929$.

[En-36] Engstrom, H.T. and Zorn, M., The Transformation of Reference Systems in the Page Relativity, Phys. Rev. 49, 701 (1936)

[Fr-05] Frenkel, J., Princípios de Eletrodinâmica Clássica, EDUSP, São Paulo, 2005.

[Fr-97] Francesco, P., Mathieu, P. and Sénéchal, D., Conformal Field Theory, Springer, New York, 1997.

[Fu-62] Fulton, T., Rohrlich, F. and Witten, L., Conformal Invariance in Physics, Rev. Mod. Phys. 34, 442, 1962.

[Fu-62a] Fulton, T., Rohrlich, F. and Witten, L., Physical Consequences of a Co-ordinate Transformation to a Uniformly Accelerating Frame, Nuovo Cimento A, 26(4), 652, 1962.

[Ga-28] Gauss, C.F., General solution of the problem: to represent the parts of a given surface on another given surface, so that the smallest parts of the representation shall be similar to the corresponding parts of the surface represented, The Philosophical Magazine and Annals of Philosophy, New Ser., IV, 104-113 e 206-215, 1828. Disponível em: Internet Archive - <http://www.archive.org/>. Acesso em 01.03.2013.

[Go-05] Gozzi, E. and Mauro, D., Scale Symmetry in Classical and Quantum Mechanics, Physics Letters A, 345, 273-278, 2005.

[Go-08] Gozzi, E. and Mauro, D., Mechanical Similarity as a Generalization of Scale Symmetry, J. Phys. A: Math and General, 39, 13, 2006.

[Go-13] Gonera, J. Conformal Mechanics, Annals of Physics, 9, 2013.

[Go-80] Goldstein, H., Classical Mechanics, $2^{\mathrm{a}}$ ed., Addison-Wesley Publishing Company, 1980 . 
[Gr-96] Greiner, W. and Reinhardt, J., Field Quantization, Springer-Verlag Berlin Heidelberg, 1996.

[Gr-99] Griffiths, D. J., Introduction to Electrodynamics, Prentice Hall, 1999.

[Gu-61] Gupta, S. N., Conformal Transformations and Space Travel, Science, 134, 1961.

[Ha-09] Halley, E., An easy demonstration of the analogy of the logarithmic tangents, to the meridian line, or sum of the secants: with various methods for computing the same to the utmost exactness, The Philos. Transact. Royal Soc. London, from their commencement, in 1665 to the year 1800, Vol. IV (1694-1702), 68-77, 1809. Disponível em: Internet Archive $<$ http://www.archive.org/>. Acesso em 01.03.2013.

[Ha-99] Hassani, S., Mathematical Physics, a Modern Introduction to its Foundations, Springer-Verlag New York, 1999.

[He-01] Henle, M., Modern Geometries, Non-Euclidean, Projective and Discrete, Prentice Hall, 2001.

[He-95] Heald, M.A. and Marion, J.B., Classical Electromagnetic Radiation, Saunders College Pub., 1995.

[Hi-45] Hill, E. L., On Accelerated Coordinate Systems in Classical and Relativistic Mechanics, Phys. Rev., 67, 1945.

[Hi-47] Hill, E. L., On the Kinematics of Uniformly Accelerated Motions and Classical Electromagnetic Theory, Physical Review , 72, 1947.

[In-52] Ingraham, R.L., Conformal Relativity, Proc. Nat. Acad. Sci. USA 38, 921, 1952.

[Ja-72] Jackiw, R., Introducing Scale Symmetry, Physics Today, 25, January, 1972.

[Ja-99] Jackson, J. D., Classical Electrodynamics, John Wiley \& Sons, 1999.

[Jo-61] Jones, R. T., Conformal Coordinates Associated with Uniformly Accelerated Motion, Am. J. Phys, 29, 2, 1961.

[Jo-98] José, J. V. and Saletan, E. J., Classical Dynamics: a Contemporary Approach, Cambridge University Press, 1998.

[Ka-08] Kastrup, H. A., On the Advancements of Conformal Transformations and their Associated Symmetries in Geometry and Theoretical Physics, Ann. Phys. 17, 631, 2008.

[Ka-62] Kastrup, H. A., Some Experimental Consequences of Conformal Invariance at Extremely High Energies, Physics Letters , 3, 2, December, 1962.

[Ka-66] Kastrup, H.A., Gauge Properties of the Minkowski Space; Phys. Rev. 150, 11831193, 1966. 
[Ka-66a] Kastrup, K.A., Position Operators, Gauge Transformations, and the Conformal Group, Physical Review, 143, 4, 1966.

[Ka-68] Kastrup, H., Gauge Properties of the Galilei Space, Nuclear Physics, B7, 545-558, North-Holland Publ. Comp., Amsterdam, 1968.

[Ku-07] Kühnel, W. and Rademacher, H. B., Liouville's Theorem in Conformal Geometry, J. Math. Pures Appl., 88, 2007.

[Ku-77] Kurak, V., Conformal Invariant Quantum Field Theory and Composite Field Operators, Revista Brasileira de Física, Vol. 7, nº 3, 1977.

[La-15] Laugel, L., Solution gènèrale de ce problème: reprèsenteres les parties d'une surface donnée sue une autre surface donnée de telle sorte que la reprèsentation soit sembleble a l'original dan les parties infiniment petites [Représentation Conforme] par C.F. Gauss, Hermann \& Fils, Paris, 1915. Disponível em: Gallica- 〈http://gallica.bnf.fr/>. Acesso em 01.03.2013.

[La-66] Landau, L.D. and Lifshitz, E.M., Mechanics (Vol. 1 of Course of Theoretical Physics), Pergamon Press, Oxford, 1960.

[La-69] Lagrange, J.L. de, Sur la Construction des Cartes Gèographiques, 637-692, GauthierVillars, Paris, 1869. Disponível em: Gallica - 〈http://gallica.bnf.fr/>. Acesso em 01.03.2013.

[La-72] Lambert, J.H., Anmerkungen und zusätze zur entwerfung der land- und himmelscharten, in: Beyträge zum Gebrauche der Mathematik und deren Anwendung durch J.H. Lambert, Mit Kupfern und Tafeln, III. Theil, Verlag der Buchhandlung der Realschule, Berlin, 105-199, 1772. Disponível em: University of Michigan Historical Math Collection, UMDL - <http://quod.lib.umich.edu/u/umhistmath/>. Acesso em 01.03.2013.

[La-99] Laugwitz, D., Riemann's Dissertation and Its Effect on the Evolution of Mathematics, The American Mathematical Monthly, Vol. 106, No. 5, Maio, 463-469, 1999.

[Le-07] Lemos, N. A., Mecânica Analítica, Editora Livraria da Física, $2^{a}$ Edição, São Paulo, 2007.

[Li-50] Liouville, J., Thèorème sur l'équation , Journ. Mathém. Pure et Appliquées 15, 103, 1850. Disponível em: Gallica - 〈http://gallica.bnf.fr/>. Acesso em 01.03.2013.

[Li-71] Lie, S., Ueber diejenige theorie eines raumes mit beliebig vielen dimensionen, die der krümmungs-theorie des gewöhnlichen Raumes entspricht, Nachr. Königl. Gesellsch. Wiss. u. d. G. - A. - Univ. Göttingen, 191-209, 1871. Disponível em GDZ - <http://gdz.sub.unigoettingen.de>. Acesso em 01.03.2013.

[Li-72] Lie, S., Ueber complexe, insbesondere linien- und kugel-complexe, mit anwendungauf die theorie partieller differentialgleichungen, Mathem. Annalen 5, 145-256, 183-186, 1872. Disponível em GDZ - 〈http://gdz.sub.uni-goettingen.de〉. Acesso em 01.03.2013.

[Li-86] Lie, S., Untersuchungen über transformationsgruppen. II, Archiv f. Mathematik og Naturvidenskab 10, 353-413, Kristiania, 1886. 
[Lo-77] Logan, J.D., Invariant Variational Principles, Mathematics in Science and Engineering, Cap. 7, Vol 138, pags 131-149, 1977.

[Lo-95] Lorch, R.P., Ptolemy and Maslama on the Transformation of Circles into Circles in Stereographic Projection; Arch. Hist. Exact Sci. 49, 271-284, 1995.

[Ma-13] Disponível em: 〈http://mathworld.wolfram.com/CylindricalProjection.html>, acesso em 01.03.2013.

[Ma-13a] Disponível em: 〈http://mathworld.wolfram.com/MercatorProjection.html>, acesso em 01.03.2013.

[Ma-13b] Disponível em: <http://mathworld.wolfram.com/LambertConformalConicProjec $\underline{\text { tion.html }}$, acesso em 01.03.2013.

[Ma-72] Maxwell, J.C., On the condition that, in the transformation of any figure by curvilinear coordinates in three dimensions, every angle in the new figure shall be equal to the corresponding angle in the original figure, Proc. London Mathem. Soc. 4, 117, 1872.

[Ma-73] Maxwell, J.C., A Treatise on Electricity and Magnetism, Clarendon Press, Oxford, 1873.

[Mc-03] McQuarrie, D. A., Mathematical Methods for Scientists and Engineers, University Science Books, 2003.

[Mo-02] Morehead, J.C. and Hiltebeitel, A.M., Karl Friedrich Gauss, General Investigations of Curved Surfaces of 1827 and 1825, The Princeton University Library, Princeton, 1902. Disponível em: Internet Archive - <http://www.archive.org/>. Acesso em 01.03.2013.

[Mo-50] Monge, G., Application de L'analyse à la Géométrie, 5. édition, revue, corrigée et annotée par M. Liouville, Bachelier, Paris, 609-616, 1850. Disponível em: Google Book Search (Harvard Library) - <http://books.google.de/>. Acesso em 01.03.2013.

[Mu-13] Disponível em: <http://catalogue.museogalileo.it/gallery/PlanisphericAstrolabe.

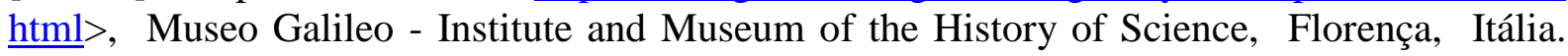
Acesso em 28.02.2013.

[Ne-49] Neugebauer, O., The Early History of the Astrolabe, Isis 40, 240-256, 1949.

[Ne-97] Needham, T., Visual Complex Analysis, Oxford University Press, 1997.

[No-18] Noether, E., Invariante Variationsprobleme $(F$. Klein zum fünfzigjährigen Doktorjubiläum.), Nachr. Königl. Gesellsch. Göttingen, Math. Physik. Klasse 1918, 235-257. Disponível em: GDZ - 〈http://gdz.sub.uni-goettingen.de>. Acesso em 01.03.2013.

[No-74] North, J.D., The Astrolabe, Scient. American, 230, 96-106, Jan., 1974.

[Pa-10] Padmanabhan, T., Gravitation Foundations and Frontiers, Cambridge University Press, 2010. 
[Pa-33] Patterson, B. C., The Origins of the Geometric Principle of Inversion, Isis, 19, 1, 1933.

[Pa-36] Page, L. and Jr, N.I.A., An Ackowledgment, Physical Review, 49, 703, 1936.

[Pe-52] Perrett, W. and Jeffery, J.B., The Principle of Relativity, Dover Publ., Inc,, New York, 1952.

[Ph-69] Phillips, R., Liouville's Theorem, Pacific Journal of Mathematics, 28, 2, 1969.

[P1-74] Plybon, B.F., Observations on the Bessel-Hagen Conservation Laws for Electromagnetic Fields, Am. J. Phys., 42, 998-1000, 1974.

[Ri-02] Rivas, M., Kinematical Theory of Spinning Particles - Classical and Quantum Mechanical Formalism of Elementary Particles, Kluwer Academic Publishers, 2002.

[Ri-06] Rindler, W., Relativity, Especial, General and Cosmological, Oxford University Press, $2^{\mathrm{a}}$ ed, 2006.

[Ri-90] Riemann, B., Grundlagen für eine allgemeine Theorie der Functionen einer veränderlichen complexen Grösse, Bernhard Riemann, Collected Papers, ed. by R. Narasimhan, Springer-Verlag, Berlin Heidelberg, p. 35-80, 1990.

[Ri-91] Rindler, W., Introduction to Special Relativity, Oxford University Press, 1991.

[Ro-08] Rowe, D. E., Einstein Studies, Volume 11: A Retrospective Review, Studies in History and Philosophy of Modern Physics, 39, 2008.

[Ro-11] Rodrigues, M.I.F., Projecções Cartográficas: Estudo Matemático das Representações Planas de uma Esfera, Dissertação de Mestrado em Matemática-Formação Contínua de Professores - Universidade do Minho - Escola de Ciências, 2011.

[Ro-72] Rosen, G., Conformal Invariance of Maxwell Equations, Am. J. Phys., 40, 1972.

[Sa-88] Saldanha, C.A.M., Sobre as Hipóteses que Servem de Fundamento à Geometria, Trans/Form/Ação (Unesp), São Paulo, 1988, vol.11, p.89-99. Tradução de: Sur les hypothêses que servent de fondement à la géométrie. Memoire de la Société Royale des Sciences de Göttingen, 1867. t. XIII, p. 272. In: Oeuvres Mathématiques de Riemann. Traduit par J. Hoüel. 2. ed. Paris, Librairie Scientifique Téchnique Albert Blanchard, 1968. p. 280 - 299.

[Se-06] Semay, C., Observer with a Constant Proper Acceleration, Eur. J. Phys. 27, 1157 2006.

[Se-78] Sergeyeva, N.D. and Karpova, L.M., Al-Farghani's Proof of the Basic Theorem of Stereographic Projection, 210-217, 1978 (apêndice 3 da referência [Th78]).

[Si-07] Sidoli, N. and Berggren, J. L., The Arabic version of Ptolemy's Planisphere or Flattening the Surface of the Sphere: Text, Translation, Commentary, SCIAMVS 8, 37-139, 2007. 
[Sn-93] Snyder, J.P., Flattening the Earth: Two Thousand Years of Map Projections, University of Chicago Press, Chicago and London, 1993.

[Sp-55] Speiser, A., L. Euler, Opera Omnia, Ser. I, 28, Orell Füssli, Zurich, 248-297, 1955.

[Ta-71] Tavel, M.A., Transport Theory and Statistical Physics, 1, 183-207, 1971 (tradução para o inglês do artigo de Emmy Noether, referência: [No-18]).

[Th-04] Thornton, S. and Marion, J., Classical Dynamics of Particles and Systems, $5^{\mathrm{a}}$ edição, Thomson, 2004.

[Th-45] Thomson, M. W., Extrait d'une lettre de M. William Thomson à M. Liouville, Journ. Mathém. Pure et Appliquées 10, 364, 1845. Disponível em: Gallica - 〈http://gallica.bnf.fr/>. Acesso em 01.03.2013.

[Th-47] Thomson, M. W., Extraits de deux lettres adressées à M. Liouville, par M. William Thomson; Journ. Mathém. Pure et Appliquées 12, 256-264, 1847. Disponível em: Gallica <http://gallica.bnf.fr/>. Acesso em 01.03.2013.

[Th-72] Thomson, W., Reprints of Papers on Electrostatics and Magnetism, Macmillan \& Co. London, 1872.

[Th-78] Thomson, R.B., Jordanus de Nemore and the Mathematics of Astrolabes: De Plana Spera, Studies and Texts 39, Pontifical Institute of Mediaeval Studies, Toronto, 1978.

[Wa-92] Warwick, A., Cambridge Mathematics and Cavendish Physics: Cunningham, Campbell and Einstein's Relativity 1905-1911 - Part I: The uses of theory, Stud. Hist. Phil. Sci., Vol. 23, No. 4, 625-656, 1992.

[We-72] Weinberg, S., Gravitation and Cosmology: Principles and Applications of General Theory of Relativity, John Wiley \& Sons, 1972.

[Wo-89] Wood, W. R., G. Papini and Y.Q. Cai, Conformal Transformations and Maximal Acceleration, Il Nuovo Cimento, 104(6), 1989.

[Wu-05] Wunsch, A. D., Complex Variables with Applications, Pearson Addison-Wesley, 2005 . 